This is the peer reviewed version of the following article: "Deciphering key intermediates in the transformation of carbon dioxide into heterocyclic products" which has been published in final form at https://www.nature.com/articles/s41929$\underline{018-0189-\mathrm{Z}}$

This article may be used for non-commercial purposes in accordance with Springer Nature Terms and Conditions for Self-Archiving.

\title{
Deciphering key intermediates in the transformation of carbon dioxide into heterocyclic products
}

Rui Huang, ${ }^{1 \#}$ Jeroen Rintjema, ${ }^{1 \#}$ Joan González Fabra, ${ }^{1}$ Eddy Martín, ${ }^{1}$ Eduardo C. Escudero-Adán, ${ }^{1}$ Carles Bo, ${ }^{1 *}$ Atsushi Urakawa, ${ }^{1 *}$ and Arjan W. Kleij ${ }^{1,2 *}$

The identification of catalytic intermediates in the conversion of carbon dioxide into is vital for improved catalyst design and optimization of structure-reactivity relationships but remains elusive. Here, we report that intermolecular hydrogen bonding (HB) interactions between an epoxy alcohol, water and the catalyst structure are crucial towards the formation of a cyclic carbonate from carbon dioxide. A combination of multiple in situ and ex situ techniques including substrate labeling, kinetic studies, computational analysis, operando infrared spectroscopy and X-ray diffraction was applied to identify and support the structural connectivities of several previously unknown intermediates. An epoxy alcohol-water cluster formed by HB was identified as the initial intermediate able to trap $\mathrm{CO}_{2}$, whereas an elusive alkyl carbonate anion could also be detected. A synergistic spectroscopic and computational analysis offers unique insight under operando conditions, and a useful analytical blueprint for key suggested intermediates in other mechanistically related $\mathrm{CO}_{2}$ conversion processes.

${ }^{1}$ Institute of Chemical Research of Catalonia (ICIQ), the Barcelona Institute of Science and Technology, Av. Països Catalans 16, 43007 Tarragona, Spain. ${ }^{2}$ Catalan Institute of Research and Advanced Studies (ICREA), Pg. Lluís Companys 23, 08010 Barcelona, Spain. \#Equal contribution, *Correspondence to cbo@iciq.cat, aurakawa@iciq.es and akleij@iciq.es. 
Catalytic $\mathrm{CO}_{2}$ activation has been recognized as a key strategy for its conversion into value-added chemicals relevant for the fine-chemical, pharmaceutical and polymer industry. ${ }^{1-6}$ The use of high-energy reactants helps to overcome the thermodynamic challenge in $\mathrm{CO}_{2}$ conversion. Such approaches are among the most popular and widely developed areas of $\mathrm{CO}_{2}$ conversion catalysis with a prominent position for both cyclic (oxetanes, aziridines, epoxides) $)^{7-9}$ as well as acyclic substrates including (homo)propargylic/allylic amines or alcohols. ${ }^{10-15}$ Most of the reported approaches towards the activation of these substrates involve the use of Lewis acid catalysts in combination with a (proposed) heteroatom based pre-activation of the $\mathrm{CO}_{2}$ molecule following cyclization towards the final product. Despite the considerable progress noted in this important area of $\mathrm{CO}_{2}$ catalysis, ${ }^{16-19}$ the determination of the exact nature of the common intermediates involved under turnover (operando) conditions poses a huge challenge.

The use of functionalized substrates, and specifically alcohol- and amine-substituted scaffolds, has expanded over the last decade. ${ }^{20}$ This has allowed for efficient and powerful substrate-controlled $\mathrm{CO}_{2}$ conversions where the functional group plays an active and decisive role in the catalytic event by producing a $\mathrm{CO}_{2}$-based nucleophile and controlling important process features such as the overall kinetics and stereoselectivity. Conventionally, Lewis acid activation of small cyclic ethers such as epoxides and oxetanes is carried out in the presence of an external nucleophile (usually a halide) to enhance the efficiency of the ring opening of the metal-bound substrate and its coupling reaction with $\mathrm{CO}_{2}$ (top of Fig. 1). There are scarce reports on epoxide/CO coupling reactions that proceed in the absence of such an external nucleophile, which offer more sustainable and attractive alternatives. ${ }^{21-22} \mathrm{~A}$ recent bimetallic oxygen-bridged $\mathrm{Al}-$ complex was shown to activate and insert $\mathrm{CO}_{2}$ into one of the $\mathrm{Al}-\mathrm{O}$ bonds in the absence of an external nucleophile as shown by NMR spectroscopy and Density Functional Theory (DFT) based studies. ${ }^{23}$ In recent work (bottom of Fig. 1), we demonstrated that $\mathrm{CO}_{2}$ can be transformed into cyclic carbonates or carbamates using epoxy alcohols or epoxy amines in the absence of nucleophile. ${ }^{7,24-25}$ We tentatively proposed the formation of an alkyl carbonate anion that acts as an in situ prepared internal nucleophile that mediates 
the ring opening of the epoxide. Such alkyl carbonic acid derived intermediates are regarded as elusive species due to their instability. ${ }^{26}$ In anionic form they have been spectroscopically and experimentally studied by trapping or stabilizing with suitable alcohol/base mixtures. ${ }^{27-31}$ However, the exact structural nature of the presumed alkyl carbonate species and how it interacts with the catalyst and medium under operando conditions remains open to debate.

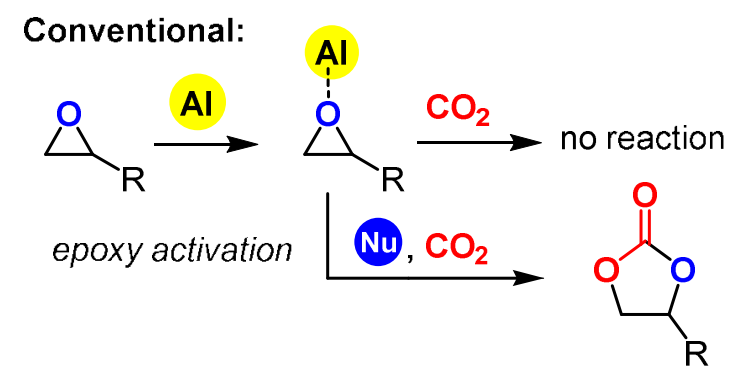

New Route:
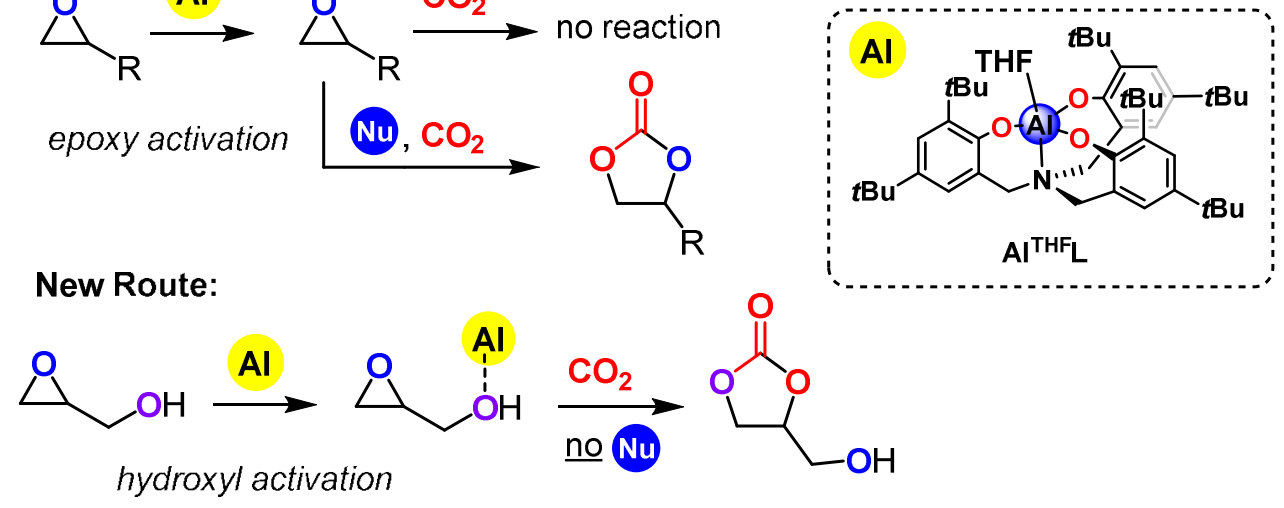

Fig. 1 | Comparison between conventional $\mathrm{C}-\mathrm{O}$ coupling of $\mathrm{CO}_{2}$ using an epoxide and a new approach using the hydroxyl group of the substrate. Al refers to an aluminum (aminotriphenolate) catalyst (yellow), Nu stands for external nucleophile. The structure of the Al-catalyst used in this work is shown at the right.

$\mathrm{HB}$ interactions have been recognized of imminent importance in the area of cyclic carbonate formation to activate the oxirane unit of the epoxide substrate towards ring opening and/or further stabilizing intermediates. ${ }^{32-35}$ Recently, Francesco and co-workers found that in situ formation of dimeric epoxy-alcohols mediated through HB is the key to facilitate the epoxide ring opening and formation of carbonate product. ${ }^{36}$ Jamison and coworkers reported that $\mathrm{HB}$ mediated by water promotes epoxide-opening cascade reactions towards the synthesis of ladder polyethers. ${ }^{37,38} \mathrm{HB}$-promoted reactions in the presence of water are often overlooked in synthetic chemistry as most transformations take place in an organic solvent, but it has gradually been realized in the last two decades that even the presence of a trace amount of water is often crucial in relation to the observed reactivity. ${ }^{39-48}$ Spectroscopic fingerprints of hydrogen-bonded water are available ${ }^{41-43}$ and the role of water in biological events was recently shown to be 
measurable through vibrational spectra. ${ }^{44-46}$ Particularly relevant to the present work is the structure of glycidol-water clusters that was investigated by a combination of vibrational spectroscopy and DFT calculations. ${ }^{47,48}$ There has been much speculation about such water clusters, especially in the context of trapping methane and other gases in water cages composed of water dodecahedrons. ${ }^{41}$

In this work, we show that the coupling of glycidol (GLY) and $\mathrm{CO}_{2}$ to afford glycidol carbonate (GLC) in the presence of an aluminum aminotriphenolate complex (see Figure 1) proceeds through trapping of $\mathrm{CO}_{2}$ by the glycidol substrate. This results in the formation of an epoxy-alcohol-water cluster that evolves into GLC with the Al-complex acting both as a proton-shuttle and as stabilizer of a crucial alkyl carbonate intermediate. This multicomponent system was examined by computational, experimental and in situ and operando IR techniques. These combined efforts provided not only a rationale for the observed reactivity in the absence of an external nucleophile but also revealed key structural information of the catalytic intermediates including a water-stabilized glycidolAl complex and an alkyl carbonate-derivative prior to formation of the cyclic carbonate product. A combined kinetic measurement and DFT/IR/X-ray analyses provided strong evidence for the intermediacy of elusive species and the importance of the alcohol function of the substrate for catalytic turnover. The consensus between the vibrational models and computational and experimental data ${ }^{49,50}$ especially in the fingerprint region $\left(1,000-2,000 \mathrm{~cm}^{-1}\right)$ provides diagnostic insight into the nature and interactions of functional groups often proposed in a wide variety of $\mathrm{CO}_{2}$ conversion reactions. ${ }^{7-15,19-25}$

\section{Results}

Reactivity and kinetic measurements. As a model substrate for our investigation, we used GLY in combination with an aluminum aminotriphenolate complex (See Fig. 1 and Supplemenatry Method 1 for its structure). To confirm whether the epoxide ring opening of GLY indeed occurs via an intramolecular attack of a nucleophile formed by activation of $\mathrm{CO}_{2}$ through the alcohol unit, we performed the deuterium labeling experiment as shown in Fig. 2A (See Supplementary Methods 2-4, and Supplementary Table 1 for further details). Under the established reaction conditions, ${ }^{24}$ the deuterated GLY (1) was exclusively converted into carbonate 5, which supports the occurrence of an 
intramolecular attack of the nucleophilic carbonate species $\mathbf{4}$ that is formed in situ. The formation of 3 via direct $\mathrm{CO}_{2}$ insertion into the epoxide 2 as a result of a classic intermolecular ring opening was not observed. ${ }^{51} \mathrm{~A}$ comparison between the conversion of a chiral and deuterated substrate (Supplementary Method 4) suggested that inversion of configuration in (S)-GLY had taken place to afford (R)-GLC without loss of stereochemistry. This implies that the intramolecular attack of the alkyl carbonate anion on the epoxide ring has clear $S_{N} 2$ character which is in line with DFT analysis of the reaction (vide infra; Fig. 3, Supplementary Method 5 and Supplementary Fig. 1).

A

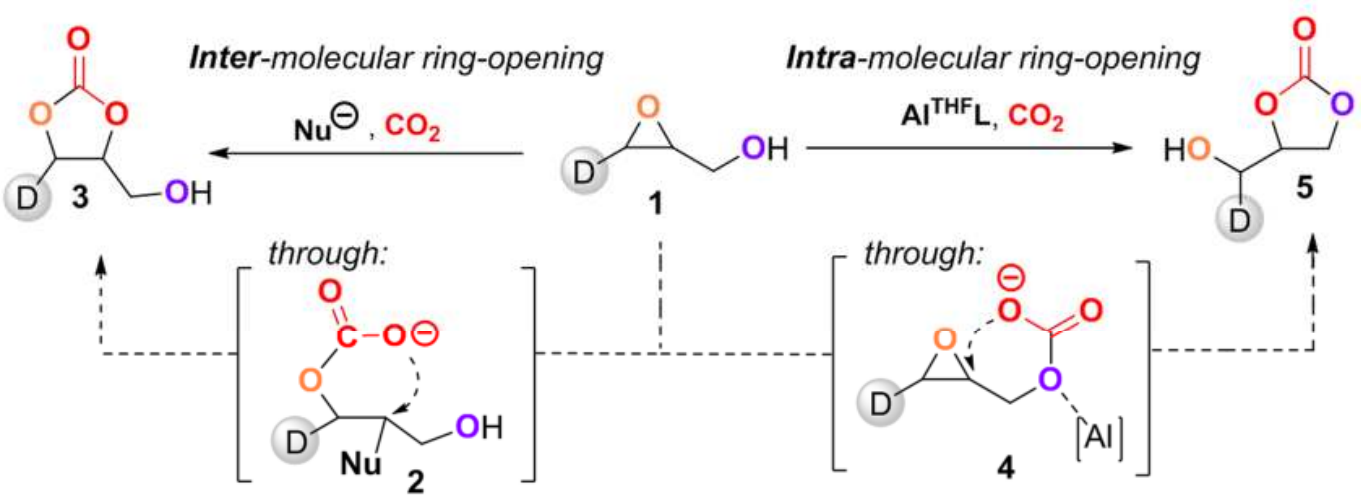

B
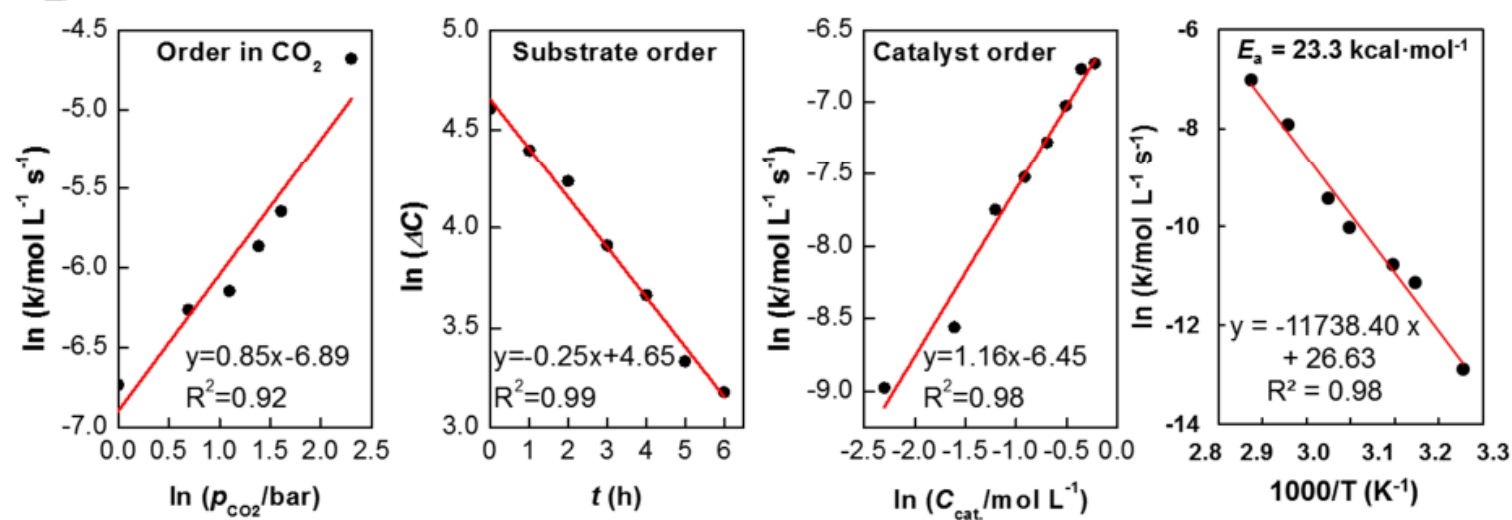

Fig. 2 | Catalytic behavior. (A) Conversion of deuterium labeled GLY 1 via intramolecular attack of the alkyl carbonate anion (4) leads to exclusive formation of 5. (B) Kinetic measurements showing the reaction order in $\mathrm{CO}_{2}$, GLY, Al-catalyst and the experimental activation energy $\left(E_{\mathrm{a}}\right)$ of the process. All data points are averaged over 2 runs.

Kinetic experiments (Supplementary Method 6) were conducted to determine the reaction order for each component. An interesting effect was observed when measuring the influence of $\mathrm{CO}_{2}$ on the reaction rate. When looking at the reaction rate at lower pressures (1-10 bar), we found a near first order in $\mathrm{CO}_{2}$ (Fig. 2B). At higher pressures 
between 10-30 bar, the solution presumably reaches $\mathrm{CO}_{2}$-saturation and the reaction rate was unaffected by the $\mathrm{CO}_{2}$ concentration, and these conditions were taken as a starting point for further kinetic experiments. At pressures $>30$ bar, the yield of GLC drops probably due to a reduced solubility of the reagents in supersaturated $\mathrm{CO}_{2}$ solution. This effect is most significant under supercritical conditions of $\mathrm{CO}_{2}\left(>73\right.$ bar, $\left.>31^{\circ} \mathrm{C}\right)$, giving only $18 \%$ conversion versus $>95 \%$ in the pressure range of 10-30 bar (Supplementary Method 6). Initially, we considered the possibility that two Al-centers are involved activating both the epoxide and alcohol moiety. However, kinetic measurements revealed an approximate first order dependence on the aluminum complex suggesting that only one molecule of catalyst is involved in the rate-determining step (Fig. 2B). GLY was previously proposed to participate in catalysis via its hydroxyl group acting as a HB donor. ${ }^{36}$ However, kinetic analysis revealed a clear first order in [GLY], indicating that it does not engage in intermolecular activation of another GLY substrate molecule in the presence of the Al complex.

Computational investigation. Bearing the first order dependence on both reactant and Al-catalyst in mind, we performed extensive DFT-based computational studies to model possible reaction mechanisms, both mono- and bi-metallic, and to investigate the stability of reaction intermediates and the energy barriers associated to their formation (see Supplementary Method 5 and Supplementary Fig. 1 for full details). The resulting most favorable reaction Gibbs energy profile on the aluminum catalyzed formation of GLC is depicted in Fig. 3, and these studies allowed us to embark on the spectroscopic determination of the key intermediates (vide infra).

The first step is coordination of GLY to the axial coordination site of the Al-catalyst forming the most stable intermediate Int1 before reaching the highest transition state. This was found to be a favorable interaction, with an unexpectedly lower energy for the coordination of GLY to the Al center via the alcohol unit (Int1) rather than the epoxy group. Deprotonation of Int1 by the ligand with a barrier of $14.5 \mathrm{kcal} \mathrm{mol}^{-1}$ leads to an alkoxide species. Initially we found a stepwise pathway for the subsequent reaction with $\mathrm{CO}_{2}$ to form a carbonate species, which ring-opens the epoxide and allows for formation of GLC. This reaction path, without water (blue line in Fig. 3), presents an overall energy barrier of $46.2 \mathrm{kcal} \cdot \mathrm{mol}^{-1}$, which is too high and not in agreement with the experimental data (23.3 
$\mathrm{kcal} \cdot \mathrm{mol}^{-1}$, see Fig. 2B). Interestingly, the involvement of catalytic amounts of water (originating from the solvent) significantly lowers the energy barrier ( $\Delta \Delta \mathrm{G}^{\ddagger}=21.8 \mathrm{kcal} \mathrm{mol}^{-}$ $\left.{ }^{1}\right)$ for the rate-determining step by facilitating $\mathrm{CO}_{2}$ insertion, proton transfer to the epoxide and ring opening in a concerted manner. This is consistent with an experimental comparison between anhydrous and normal conditions (32\% vs $62 \%$ GLC yield respectively, Supplementary Fig. 2). It should be further noted that under the experimental conditions used, no hydrolysis of the GLY to glycerol was observed (see also Supplementary Table 2).

The insertion of $\mathrm{CO}_{2}$ and ring opening of the epoxide in the presence of water takes thus place via a concerted mechanism (green trace in Fig. 3). It starts with stabilization of the alkoxide species incorporating one molecule of $\mathrm{H}_{2} \mathrm{O}$ and $\mathrm{CO}_{2}$ to form the relatively low-barrier ensemble intermediate (Int2). C-O bond formation between the alkoxide and $\mathrm{CO}_{2}$ leads to intermediate Int3 with a chelating hemi-ester of a carbonic acid anion. Finally, the second transition state with the highest energy barrier is reached by epoxide ring opening via a concerted mechanism, leading to formation of the Al-bound cyclic carbonate (Int4/Int4') followed by the release of the free product (GLC).

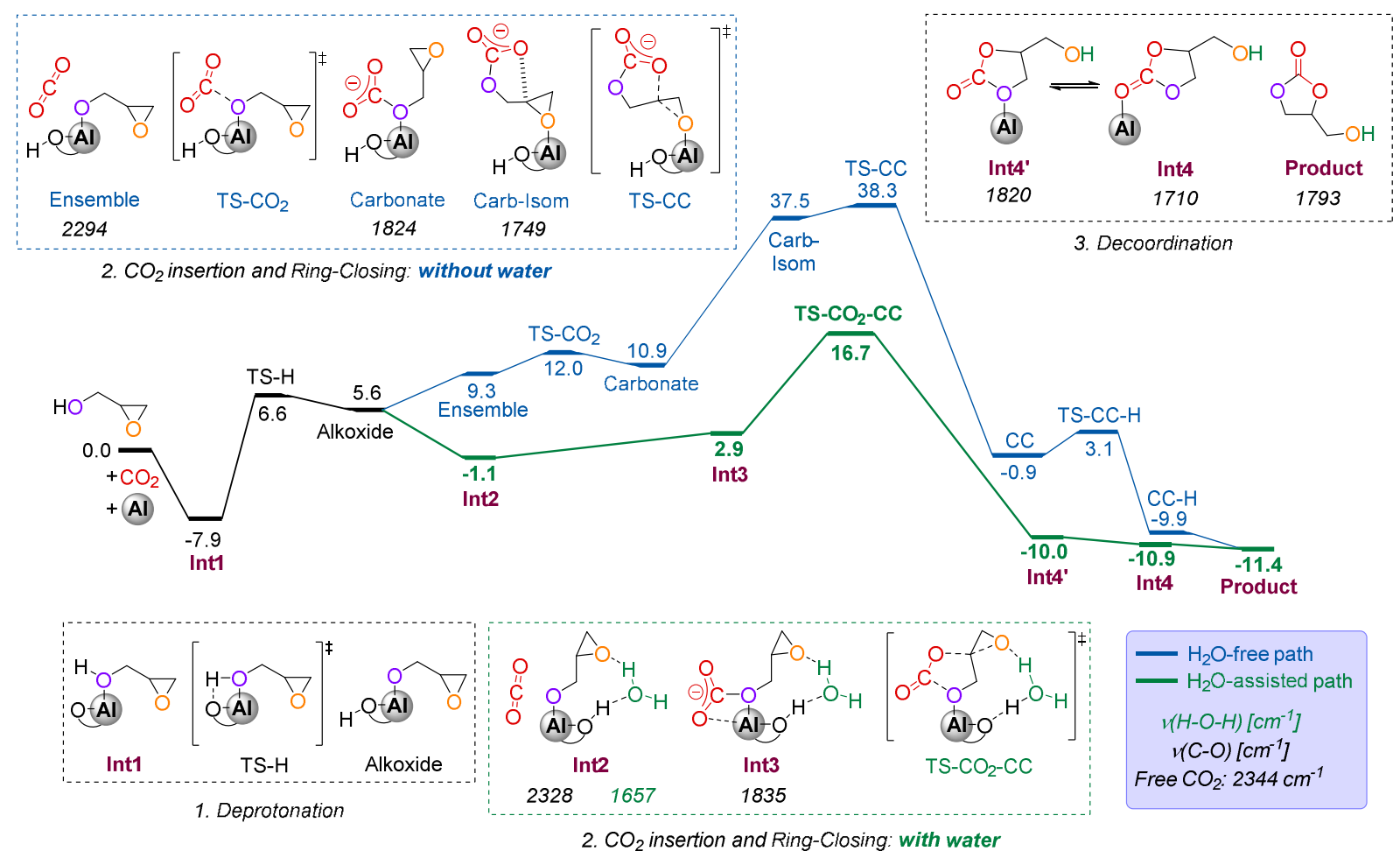


Fig. 3 | Gibbs free energy (B97-D3, $\mathrm{kcal} \cdot \mathrm{mol}^{-1}$ ) profiles. GLC is formed with a rate-determining step through a $\mathrm{H}_{2} \mathrm{O}$-assisted (green line) and $\mathrm{H}_{2} \mathrm{O}$-free (blue line) routes. The notation for the key intermediates are highlighted in purple. Schematic structures of the intermediates and transition states at the different steps of the reaction are illustrated within dashed boxes with the IR-active frequency of the characteristic band for these key intermediates. See Supplementary Tables S3-5 and Supplementary Discussions 1-5 for further computational details.

The activation energy of the overall reaction determined experimentally was 23.3 $\mathrm{kcal} \cdot \mathrm{mol}^{-1}$ (Fig. 2B), which is in fair agreement with the DFT-computed value of 24.6 $\mathrm{kcal} \cdot \mathrm{mol}^{-1}$. An intermolecular case where a bicarbonate anion formed by $\mathrm{CO}_{2}$ and traces of $\mathrm{H}_{2} \mathrm{O}$ triggers the ring opening of propylene oxide (PO) has been reported by Ema, with a reduction of $12 \mathrm{kcal} \cdot \mathrm{mol}^{-1}$ in the calculated activation energy compared to the waterfree case. ${ }^{51}$ However, we did not observe any beneficial effect towards GLY conversion upon adding an external bicarbonate species (Supplementary Table S1), implying that free bicarbonate species are likely spectators under our experimental conditions. Contrarily, a favorable intramolecular attack in the alkyl carbonate anion transition state (Int3) with $\mathrm{H}_{2} \mathrm{O}$ facilitating proton transfer leads to a lower energy barrier in this ratedetermining step. However, the current experimental data discussed so far is insufficient to support the existence of these calculated intermediates under the experimental conditions. Therefore, in order to gather further experimental support for the reaction pathway proposed by our DFT calculations, in situ ATR-IR spectroscopy in the solid and liquid state as well as operando high-pressure transmission IR spectroscopy were used to scrutinize the coordination environment of the Al center and the key interactions between the reaction components as presented below.

Solid-state ATR-IR spectroscopy. The structure of the Al-catalyst (AI THFL, Figure 1) was previously determined by $X$-ray analysis, ${ }^{52}$ showing the disposition of $L$ and an axial THF ligand coordinating to the Al center (Fig. 4). For precise comprehension of the vibrational fingerprints involving the $\mathrm{Al}$ site and intermolecular interactions with substrates, experimental and theoretical IR spectra of the Al complex with THF (AITHFL) and without THF $(\mathrm{Al}(\mathrm{L}))$ were compared for unambiguous assignments (Fig. 4A, Supplementary 
Videos 1-6). Details of the synthesis of $\mathrm{Al}(\mathrm{L})$ and $\mathrm{Al}{ }^{\mathrm{THF}} \mathrm{L}$ are provided in Supplementary Method 1.

The comparison shown in Fig. 4A reveals characteristic spectral features induced by the coordinative interaction between the $\mathrm{Al}$ center and the O-atom of THF, clarifying the structural changes within the THF (C-O-C stretching band of THF (Peak3), Table 1) and $\mathrm{Al}(\mathrm{L})(\mathrm{C}-\mathrm{N}$ stretching band (Peak2) and Al-O stretching band of $\mathrm{Al}(\mathrm{L})$ (Peak4), Table 1). The bands of non-interacting $\mathrm{C}-\mathrm{H} / \mathrm{C}-\mathrm{C}$ fragments of the ligand (for example, Peak6, Table 1 and other unlabeled vibrations) remain unchanged. These spectral changes are perfectly reproduced by the predicted IR shifts upon coordination of THF to the Al center (Fig. 4A). Based on these vibrational features, interactions between $\mathrm{GLY}$ and $\mathrm{Al}(\mathrm{L})$ were studied by varying the amount of $\mathrm{GLY}$ with respect to $\mathrm{Al}(\mathrm{L})$ (Fig. 4B, Table 1). The major spectral changes are similar to those observed for THF coordination to $\mathrm{Al}(\mathrm{L})$ (Table 1) including a blue-shift of the $\mathrm{C}-\mathrm{N}$ vibration (Peak2, $\mathbf{C}-\mathbf{N}$ ), a red-shift of the Al-O-Ph vibration (Peak4) and the appearance of an Al-GLY vibration (Peak5), confirming the coordination of GLY to the Al center. The C-OH stretching band of GLY underwent a redshift from 1035 (unbound state) to $1008 \mathrm{~cm}^{-1}$ (Peak 3), while the frequency of the $\mathrm{C}-\mathrm{O}-$ C stretching band $\left(901 \mathrm{~cm}^{-1}\right)$ remained unchanged. $A$ further increase in the relative amount of $\mathrm{GLY}$ to $\mathrm{Al}(\mathrm{L})$ from 1 to 2 molar equivalents resulted in an enhancement of the band attributed to the $\mathrm{C}-\mathrm{OH}$ vibration of unbound GLY (Fig. 4B). These results show that GLY binds to the Al center through the oxygen atom of the $\mathrm{OH}$ group in a 1:1 stoichiometry. These data are fully in line with the DFT predicted initial coordination of GLY to the Alcomplex (Int1).

A series of control experiments were conducted to compare the interactions of $\mathrm{Al}(\mathrm{L})$ with GLY and other O-containing molecules using the same ATR-IR method (Supplementary Fig. 3). The results show that $\mathrm{Al}(\mathrm{L})$ is able to coordinate both the alcohol group of isopropanol and epoxy group of propylene epoxide (PO), but it prefers to coordinate via the hydroxyl moiety (GLY) when both functional groups are present in the same molecule. In addition, no changes in the bonding environment of $\mathrm{Al}(\mathrm{L})$ were observed when it was treated with $\mathrm{CO}_{2}$ and $\mathrm{H}_{2} \mathrm{O}$ under ambient conditions. These observations support that activation of the alcohol group in GLY by the Al-complex is the first step in the catalytic cycle, which is distinctively different from well-known Al-based 
epoxy activation ${ }^{52,53}$ and $\mathrm{Al}-$ mediated $\mathrm{CO}_{2}$ activation under harsh conditions $\left(50 \mathrm{bar} \mathrm{CO}_{2}\right.$, $50-100{ }^{\circ} \mathrm{C}$ ) reported by North. ${ }^{23}$ This unusual alcohol activation mode was further confirmed in the solid state by X-ray analysis of the AIGLYL complex, showing that GLY is indeed coordinated to the metal via the hydroxyl group (see Int1 in Fig. 4B and X-ray structure in Supplementary Fig. 4). The crystallographic analysis thus aligns well with the intermediate species Int1 computed by DFT and the ATR-IR changes when combining $\mathrm{Al}(\mathrm{L})$ with $\mathrm{GLY}$.
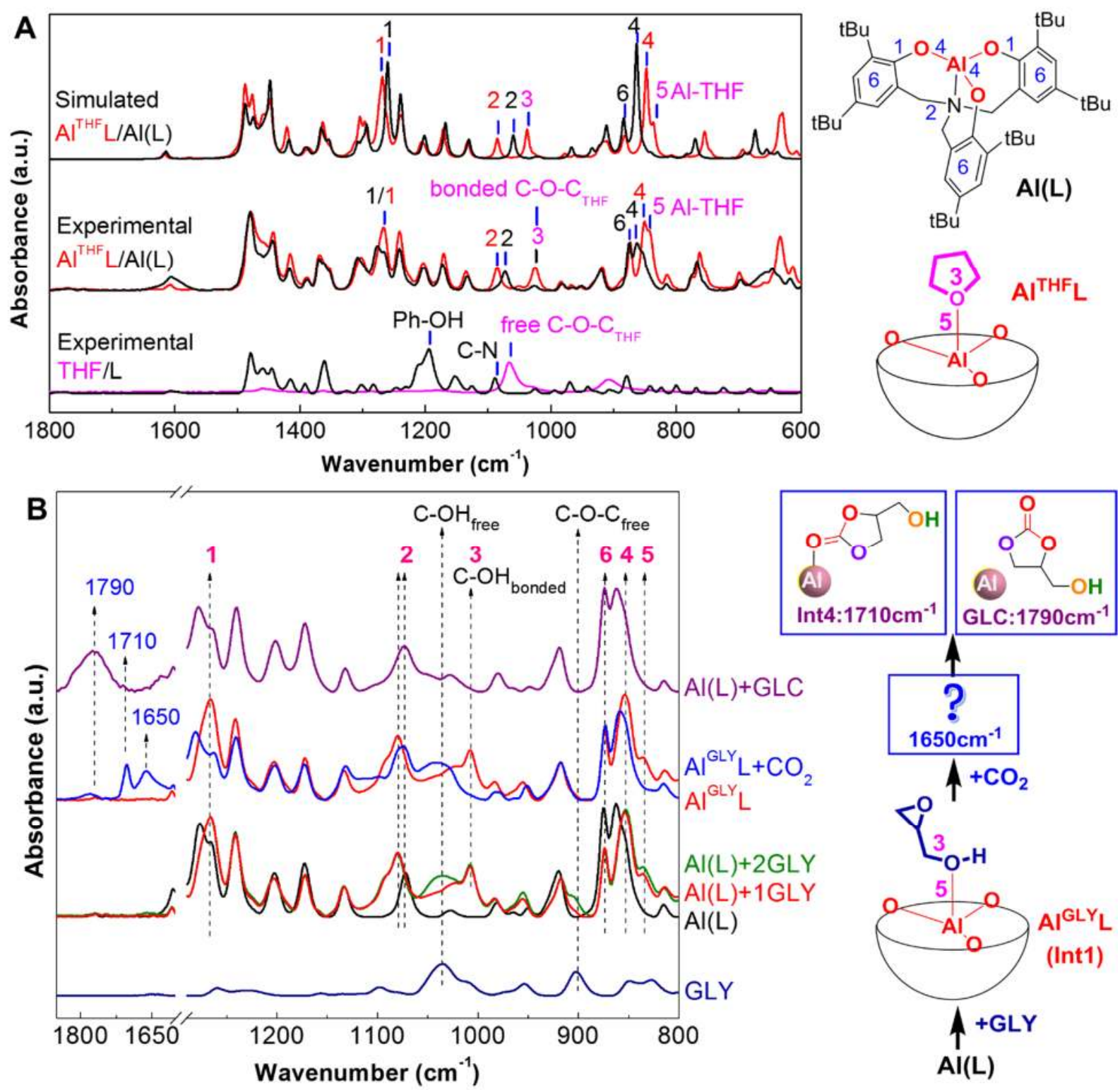

Fig. 4 | Solid-state ATR-IR spectroscopy. (A) Overlay of simulated (top) and experimental (middle) ATRIR spectra of the aluminum aminotriphenolate complex with $\left(\mathrm{Al}^{\mathrm{THF}} \mathrm{L}\right)$ and without $[\mathrm{AI}(\mathrm{L})]$ axial ligand. The 
spectra of aminotriphenolate ligand (L) and THF are shown as reference (bottom). The experimental spectra were collected as the as-synthesized solid materials. The numbers indicated on some bands correspond to the characteristic vibrational modes of the chemical moiety with respective numbering as shown in the chemical structures. (B) ATR-IR spectroscopic analysis of the interactions of the Al-complex $[A I(L)]$ with glycidol (GLY, 1:1 or 1:2 molar ratio), glycidol carbonate $(G L C)$ and the interactions of $A I^{G L Y} L$ at 30 bar $\mathrm{CO}_{2}$ at $25{ }^{\circ} \mathrm{C}$ after 2 weeks. The X-ray crystal structure of $\mathrm{Al}^{\mathrm{GLY} L}$ can be found in Supplementary Fig. 4. The vibrational frequencies of the highlighted bands are summarized in Table 1.

Table 1 | Experimental vibrational frequencies $\left(\mathrm{cm}^{-1}\right)$ of the characteristic IR bands

\begin{tabular}{|c|c|c|c|c|c|c|c|}
\hline \multirow{2}{*}{ Sample } & \multirow{2}{*}{$\begin{array}{l}\text { Peak1 } \\
v(\text { Ph-O) }\end{array}$} & \multirow{2}{*}{$\begin{array}{l}\text { Peak2 } \\
v(C-N)\end{array}$} & \multicolumn{2}{|c|}{ Peak3 } & \multirow{2}{*}{$\frac{\text { Peak4 }}{\mathrm{v}(\mathrm{Al}-\mathrm{O})_{\mathrm{Ph}}}$} & \multirow{2}{*}{$\frac{\text { Peak5 }}{v(\mathrm{Al}-\mathrm{O})_{\text {THF/GLY }}}$} & \multirow{2}{*}{$\begin{array}{c}\text { Peak6 } \\
\delta(\mathrm{C}-\mathrm{H} / \mathrm{C}-\mathrm{C}\end{array}$} \\
\hline & & & $v(C-O-C)$ & $v(\mathrm{C}-\mathrm{OH})$ & & & \\
\hline$\overline{A l^{\mathrm{THF}} \mathbf{L}}$ & 1267 & 1086 & $1025(1066)$ & - & 850 & 837 & 874 \\
\hline $\mathrm{Al}(\mathrm{L})$ & 1267 & 1072 & - & - & 863 & - & 874 \\
\hline$A l^{G L Y} \mathbf{L}$ & 1267 & 1080 & $901(901)$ & $1008(1035)$ & 854 & 835 & 874 \\
\hline $\mathrm{Al}^{\mathrm{GLY}} \mathrm{L}+\mathrm{CO}_{2}$ & 1267 & 1072 & - & - & 863 & - & 874 \\
\hline
\end{tabular}

For Peak3, the frequency values in/outside the brackets denote the values without/with coordination to Al, respectively

To understand the subsequent catalytic reaction steps, the first intermediate species (Int1, $\mathrm{Al}{ }^{G L Y} \mathrm{~L}$ ) in its solid form was treated under 30 bar of $\mathrm{CO}_{2}$ at room temperature for two weeks and studied by ATR-IR (Fig. 4B). After the $\mathrm{CO}_{2}$ treatment, the GLY moiety in $\mathrm{Al}^{\mathrm{GLY}} \mathrm{L}$ was converted to some carbonyl species as confirmed by the disappearance of the bands arising from the coordination of the $\mathrm{C}-\mathrm{OH}$ fragment to the $\mathrm{Al}$ center (Peak3) and Al-GLY (Peak5). Moreover, new bands appeared in the range 1650-1790 $\mathrm{cm}^{-1}$. The $\mathrm{Ph}-\mathrm{O}$ band (Peak1) decreased to the same intensity as that of $\mathrm{Al}(\mathrm{L})$, indicative of the structural recovery of $\mathrm{Al}(\mathrm{L})$ after conversion of GLY. The shift of the $\mathrm{C}-\mathrm{N}$ vibration (Peak2) and $\mathrm{Al}-\mathrm{O}-\mathrm{Ph}$ bands (Peak4), similar to the transformation of $\mathrm{Al}^{\mathrm{THF}} \mathrm{L}$ to $\mathrm{Al}(\mathrm{L})$ (see Fig. 4A), also indicated transformation of GLY in AIGLY . The new band at $1790 \mathrm{~cm}^{-1}$ corresponds to the carbonate product (GLC). ${ }^{54,55}$ Importantly, the band at $1710 \mathrm{~cm}^{-1}$ was not present for the unbound GLC molecule and it was attributed to GLC bound to the Al-complex as illustrated in Fig. 4B. Such a suggested structure and its characteristic vibrational frequency are consistent with Int4 proposed by the DFT calculations (Fig. 3). The spectroscopic features of $\mathrm{Al}(\mathrm{L})$ upon external addition of $\mathrm{GLC}$ (Fig. 4B, $\mathrm{Al}(\mathrm{L})+\mathrm{GLC}$ ) shows 
a detectable band around $1710 \mathrm{~cm}^{-1}$ supporting the view of the intermediacy of $A I^{G L C L}$. The reduced nature of this band may be rationalized by competitive coordination of the alcohol group of GLC to $\mathrm{Al}(\mathrm{L})$, as observed for $\mathrm{GLY}$ in $\mathrm{Al} \mathrm{ILY}^{\mathrm{L}}$ (Int1).

The emerging band at $1650 \mathrm{~cm}^{-1}$ after the $\mathrm{CO}_{2}$ treatment (Fig. 4B) was initially ascribed to the Carbonate species of the water-free route in the DFT calculations (Fig. 3). However, this and other intermediates (Figure 3 and Table S4) do not show a band near $1650 \mathrm{~cm}^{-1}$ except for Int2 present in the $\mathrm{H}_{2} \mathrm{O}$-assisted reaction path. Further investigation clarified that the broad band near $1650 \mathrm{~cm}^{-1}$ may arise from the $\mathrm{O}-\mathrm{H}$ bending and $\mathrm{HB}$ interaction between GLY and water, which was confirmed by the use of $\mathrm{D}_{2} \mathrm{O}$ (Supplementary Fig. 5). This is consistent with the previous observations of the formation of stable GLY-water clusters. ${ }^{47,48}$ Such GLY-water clusters show similarities with that of the $\mathrm{OH}$ moiety of Int2 in the DFT calculations (Fig. 3). At this point, however, there was no evidence for the interaction between $\mathrm{CO}_{2}$ and the Al-complex within such an ensemble, and further IR studies were performed to gain more precise information regarding the ensemble generated in a reactive solution environment.

In situ ATR-IR spectroscopy in solution. Ensembles of GLY-water characterized by the band near $1650 \mathrm{~cm}^{-1}$ were also observed under diluted conditions in an apolar solvent, similar to that of GLY-water in the solid state (Supplementary Fig. 5, Supplementary Discussion 6 and Fig. 4B). The comparison between GLY (Supplementary Fig. 5) and another epoxy alcohol (3,4-epoxy-1-butanol) having a longer alkyl alcohol side chain (Fig. 5 ) shows that the IR signal of such ensembles increases with the alcohol chain length further emphasizing the importance of the alcohol moiety.

The interaction of the latter epoxy alcohol with the other components present in the reaction mixture was further investigated using a set of specific sequences to identify the role of the epoxy alcohol-water ensembles under turnover conditions (Fig. 5). The bottom spectrum shows that the ensemble $\left(1650 \mathrm{~cm}^{-1}\right)$ can be consistently generated from the epoxy alcohol and trace amounts of adventitious water present in the medium. To our surprise, upon changing the atmosphere from $\mathrm{Ar}$ to $\mathrm{CO}_{2}$, the intensity of this band as well as those of epoxy alcohol in the lower frequency region (Supplementary Fig. 5) was drastically enhanced. Even after the removal of $\mathrm{CO}_{2}$ from the solution by carefully flushing with $\mathrm{Ar}$ (evident from the full disappearance of the band of dissolved $\mathrm{CO}_{2}$ at $c a .2340 \mathrm{~cm}^{-}$ 
1) the band positions remained nearly unaltered with only minor intensity changes. Subsequently, adding a nucleophile (TBAl: tetrabutylammonium iodide, Fig. 5A) to this solution led to the formation of the cyclic carbonate $\left(1790 \mathrm{~cm}^{-1}\right)$ even in the absence of dissolved $\mathrm{CO}_{2}$ in the reaction mixture. The formation of the cyclic carbonate product could be further enhanced by addition of Al ${ }^{T H F} \mathrm{~L}$. These results evidence that $\mathrm{CO}_{2}$ is efficiently trapped in the ensemble prior to formation of the cyclic carbonate. Also, the $\mathrm{CO}_{2}$ containing ensemble interacts intermolecularly such that the dynamic dipoles of the $\mathrm{OH}$ bending (of water and the epoxy alcohol; $1650 \mathrm{~cm}^{-1}$ ) and of various vibrational modes of the epoxy alcohol are enhanced. Intriguingly, there was no indication of $\mathrm{C}=\mathrm{O}$ stretching bands due to trapped $\mathrm{CO}_{2}$. However, a careful examination of the lower frequency region (Supplementary Fig. 6) shows the emergence of a band near $1330 \mathrm{~cm}^{-1}$ when $\mathrm{CO}_{2}$ is trapped. This vibrational frequency is similar to that of bicarbonate interacting with $\mathrm{H}_{2} \mathrm{O}$ molecules. ${ }^{56}$ Thus, we conclude that $\mathrm{CO}_{2}$ forms a dynamic ensemble with the epoxy alcohol and water through a strong HB network forming stable bicarbonate species and assembling these three components as an ensemble. It is noteworthy that the trapping of $\mathrm{CO}_{2}$ could only be observed for epoxy alcohols but not for hydroxyl-free epoxides such as propylene oxide and methyl-protected GLY, or the AITHFL catalyst (Supplementary Fig. 7). The presence of the alcohol group is thus essential to form the observed ensemble.

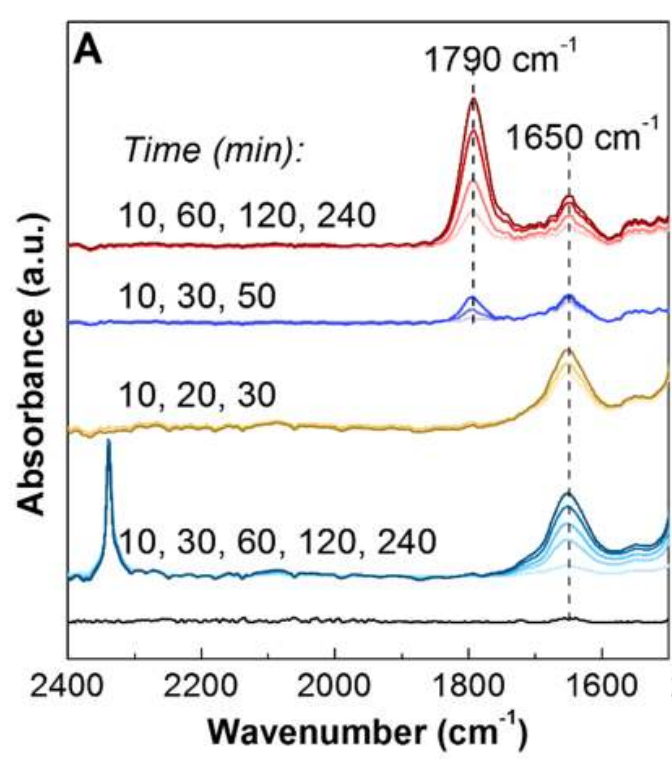

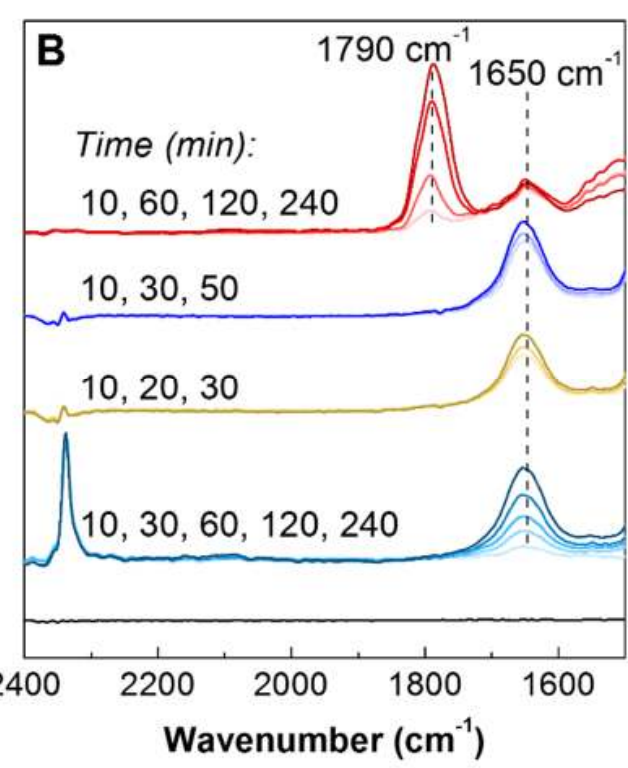

Wavenumber $\left(\mathrm{cm}^{-1}\right)$
Sequences

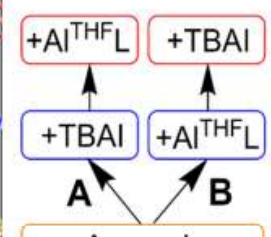

Ar purging

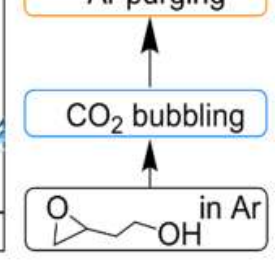


Fig. 5 | Special sequence of ATR-IR measurements in solution. Conditions: $25^{\circ} \mathrm{C}, 20 \mathrm{mmol} \cdot \mathrm{L}^{-1}$ solution of 3,4-epoxy-1-butanol in $5.0 \mathrm{~mL}$ of cyclohexane was kept under $\mathrm{Ar}$ (balloon) for $30 \mathrm{~min}$, and then $\mathrm{CO}_{2}$ was introduced into the reactor by using a $\mathrm{CO}_{2}$ balloon. After $240 \mathrm{~min}$, the free $\mathrm{CO}_{2}$ was removed by purging with Ar for 30 min. Finally, TBAl and Al ${ }^{T H F} L$ were successively added to the solution under Ar. The difference between $(A)$ and $(B)$ is the addition order of TBAl and Al ${ }^{T H F} \mathrm{~L}$. In both cases, the spectrum of pure cyclohexane was used as a background for each collection.

Comparison between Fig. 5A and 5B highlights the importance of the sequence for TBAI and $A I^{T H F} L$ addition to the ensemble species, and it shows that formation of the cyclic carbonate product can occur by addition of TBAI to this epoxy alcohol-water- $\mathrm{CO}_{2}$ ensemble based medium under ambient conditions. A subtle increase in the intensity of the band at $1650 \mathrm{~cm}^{-1}$ was observed when $A I^{T H F} L$ was added to the ensemble (Fig. 5B), indicative of an interaction with $A I^{T H F} L$ and further enhancing the dynamic dipole of the ensemble. Thus, it seems that the Al complex interacts and stabilizes the epoxy alcoholwater- $\mathrm{CO}_{2}$ ensemble, facilitating the formation of Int2 and Int 3 as determined by the DFT calculations (Fig. 3). The catalyst plays a dual role: coordinating and stabilizing the deprotonated epoxy alcohol to the Al center and having one of the phenolate arms acting as a non-innocent ligand in this proton-shuttling process (Supplementary Discussion 7).

Operando high-pressure (HP) transmission IR spectroscopy. The aforementioned ATR-IR spectra were collected under milder conditions $\left(25^{\circ} \mathrm{C}\right.$, up to $10 \mathrm{bar}$; for the reactor set up see Supplementary Fig. 8) compared to the actual reaction conditions $\left(75^{\circ} \mathrm{C}, 10\right.$ bar), with the aim to understand the intermolecular interactions among the chemical components of the reaction mixture. Under these conditions, however, it is often difficult to provide enough energy to overcome the barriers associated with the transitional intermediates forming late-stage species. Indeed, we did not find any species preceded by high energy-barriers (Fig. 3) or final products in the ATR-IR measurements under ambient conditions, except when applying more forcing conditions (i.e., high $\mathrm{CO}_{2}$ pressure combined with a two-week reaction time reported in Fig. 4B, or adding external nucleophile as shown in Fig. 5). As such, operando HP-IR was performed to find evidence for the later-stage species and to monitor real-time changes under more realistic experimental conditions $\left(75^{\circ} \mathrm{C}, 10\right.$ bar; Fig. 6$)$ in the absence of the nucleophile (TBAI). 
A mixture of $A l^{T H F} L$ and GLY, that was proven to evolve into Al-bound GLY (Int1 in Fig. 3 and 4) by ATR-IR measurements, was used as the background for the following measurements (for the reactor set up see Supplementary Fig. 9). When $\mathrm{CO}_{2}$ was introduced into the mixture, the interactions between $\mathrm{CO}_{2}$ and Int1 led to the emergence of new bands at 1837, 1159 and $1063 \mathrm{~cm}^{-1}$. This chemical state is obviously different from the HB ensemble previously observed by the low-pressure ATR-IR measurements, probably because of its rapid conversion under these more forcing conditions. A new intermediate was observed with a band at $1837 \mathrm{~cm}^{-1}$ that was not observed at ambient temperature. Further hints for the structure of this intermediate were obtained by a set of computed IR spectra that revealed that Int3 has a matching $\mathrm{C}=\mathrm{O}$ stretching frequency at $1835 \mathrm{~cm}^{-1}$ (Fig. 3, see Supplementary Table 5 for more information). Over time, formation of GLC was observed as the intensity of the GLC band at $1790 \mathrm{~cm}^{-1}$ increased along with the appearance of a third peak at $1740 \mathrm{~cm}^{-1}$. The latter does not disappear even after the reaction is finished and is attributed to coordination of the final product to $\mathrm{Al}(\mathrm{L})$ (Int4, Fig. 6). A control experiment combining Al ${ }^{\mathrm{THF}} \mathrm{L}$ with GLC (top of Fig. 6) where the Al complex and product were mixed in a 1:4 ratio under similar reaction conditions $\left(75^{\circ} \mathrm{C}, 10\right.$ bar $\left.\mathrm{N}_{2}\right)$ shows the band at $1740 \mathrm{~cm}^{-1}$, confirming that it indeed arises from a GLC-Al interaction.

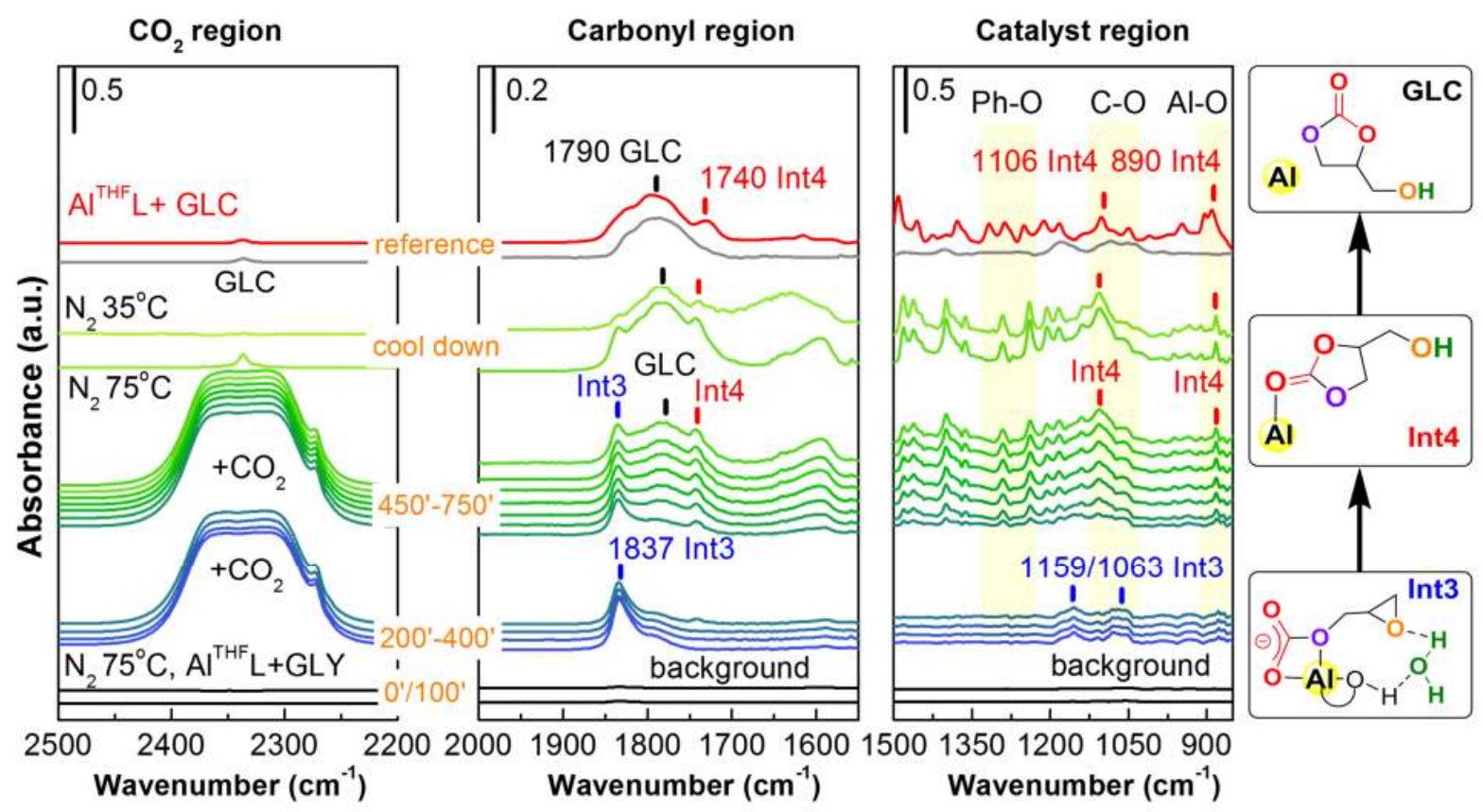


Fig. 6 | Operando HP-IR spectroscopic analysis of the reaction between $A I^{\mathrm{THF}} \mathrm{L}, \mathrm{GLY}$ and $\mathrm{CO}_{2}$. The reaction sequences were set in this order: (1) the initial reaction solution with $10 \mathrm{mmol} \cdot \mathrm{L}^{-1}$ of $A I^{T H F L}$ and 40 mmol. $\mathrm{L}^{-1}$ of $\mathrm{GLY}$ in $18 \mathrm{~mL}$ of $\mathrm{CCl}_{4}$ was measured at $75^{\circ} \mathrm{C}$ under 10 bar of $\mathrm{N}_{2}$, and this spectrum was used as background; (2) after $100 \mathrm{~min}$, the system was pressurized with $\mathrm{CO}_{2}$ at 10 bar and the reaction was followed from 200 to $750 \mathrm{~min}$; (3) finally, the reaction was terminated by cooling down to $35^{\circ} \mathrm{C}$ under $\mathrm{N}_{2}$. The spectra of GLC and an AITHFL/GLC $\left(20 \mathrm{mmol} \cdot \mathrm{L}^{-1}\right)$ mixture under the same conditions are shown as reference (top).

The intensity of the band at $1837 \mathrm{~cm}^{-1}$ assigned to Int3 was almost constant throughout the reaction, and at the initial $\mathrm{N}_{2}$-purging stage (Fig. 6). On the other hand, when $\mathrm{N}_{2}$ purging in the solution was complete (as evident from the absence of dissolved $\mathrm{CO}_{2}$ at $2340 \mathrm{~cm}^{-1}$ ) the intensity of this band started to diminish. This implies that a certain $\mathrm{CO}_{2}$ concentration in the solution is required to stabilize the formation of Int3, which is a reasonable assumption since the dissociated form (Int2) is energetically more stable (Fig. 3). The constant concentration of Int3 in comparison to GLC (Supplementary Discussion 8) can be explained by the limited conversion of GLY in our study (ca. $40 \%$ ) and also by the strong binding of GLY by the catalyst through the ensemble species. The above described in situ and operando spectroscopic results clearly illustrate that the $\mathrm{CO}_{2}$ trapped ensemble interacts strongly with the Al catalyst under the reaction condition, and forms the kinetically favored intermediate Int3 prior to its transformation to GLC.

\section{Conclusion}

In conclusion, we have demonstrated through a combination of kinetic measurements, DFT calculations, $X$-ray analysis, and multimodal spectroscopic techniques that elusive intermediates in important $\mathrm{CO}_{2}$ transformation reactions can be experimentally trapped and identified under turnover conditions. Structural information for four key intermediates was obtained from combined experimental studies, and these data is in full accordance with the computational results. The epoxy alcohol/water cluster mediated by HB interactions was identified as a critical intermediate to trap $\mathrm{CO}_{2}$ resulting in the formation of an epoxy alcohol/water/ $\mathrm{CO}_{2}$ intermediate and subsequent carbonate formation through an elusive alkyl carbonate under catalytic conditions. Multimodal spectroscopic techniques were used as nondestructive and versatile tools, allowing us to examine each key intermediate during the reaction and to analyze these step-by-step. Our results are 
expected to build a general spectroscopic and computational approach for the detailed mechanistic investigation of multiple-component solution systems that are often operative in various $\mathrm{CO}_{2}$ conversion reactions. 


\section{Methods}

Synthesis of $A^{T H F} L: A$ typical procedure uses a solution of the aminotriphenolate ligand precursor $\mathbf{L}\left(\mathrm{H}_{3}\right)$ $(1.0 \mathrm{~g}, 1.5 \mathrm{mmol})$ dissolved in THF was added a stoichiometric amount of $\mathrm{AlMe}_{3}(2 \mathrm{M}$ in heptane, $0.75 \mathrm{~mL}$, $1.5 \mathrm{mmol}$ ). The reaction mixture was stirred at room temperature for $2 \mathrm{~h}$ after which the solvent was removed in vacuo and hexane was added to precipitate the product that was isolated by filtration and carefully dried. This procedure yields $\mathbf{A l}^{\mathrm{THF}} \mathbf{L}$ as a white powder in typically $>80 \%$ yield. ${ }^{52}$

Synthesis of deuterated glycidol: The preparation and full analysis of the deuterated glycidol presented in Fig. 2, its precursors and the resultant cyclic carbonate after treatment with $\mathbf{A l}^{\mathrm{THF}} \mathbf{L}$ under an atmosphere of $\mathrm{CO}_{2}$ are reported in Supplementary Methods 7 and 8.

Data availability. A data set collection of input files and computational results is available in the ioChem$\mathrm{BD}$ repository ${ }^{57}$ and can be accessed via http://dx.doi.org/10.19061/iochem-bd-1-58. The data that support the plots within this paper and other findings of this study are available from the corresponding author upon reasonable request. CCDC 1850585 contains the supplementary crystallographic data for this paper. The data can be obtained free of charge from The Cambridge Crystallographic Data Centre via www.ccdc.cam.ac.uk/structures.

\section{References}

1. Liu, Q., Wu, L., Jackstell, R. \& Beller, M. Using carbon dioxide as a building block in organic synthesis. Nat. Commun. 6, 5933 (2015).

2. Wang, W.-H., Himeda, Y., Muckerman, J. T., Manbeck, G. F. \& Fujita, E. $\mathrm{CO}_{2}$ Hydrogenation to formate and methanol as an alternative to photo- and electrochemical $\mathrm{CO}_{2}$ reduction. Chem. Rev. 115, 1293612973 (2015).

3. Wang, W., Wang, S., Ma, X. \& Gong, J. Recent advances in catalytic hydrogenation of carbon dioxide. Chem. Soc. Rev. 40, 3703-3727 (2011).

4. Artz, J., Müller, T. E., Thenert, K., Kleinekorte, J., Meys, R., Sternberg, A., Bardow, A. \& Leitner, W. Sustainable conversion of carbon dioxide: An integrated review of catalysis and life cycle assessment. Chem. Rev. 118, DOI: 10.1021/acs.chemrev.7b00435 (2018).

5. Álvarez, A., Borges, M., Corral-Pérez, J. J., Olcina, J. G., Hu, L., Cornu, D., Huang, R. \& Urakawa, A. $\mathrm{CO}_{2}$ activation over catalytic surfaces. ChemPhysChem 18, 3135-3141 (2017).

6. Decortes, A., Castilla, A. M. \& Kleij, A. W. Salen-complex-mediated formation of cyclic carbonates by cycloaddition of $\mathrm{CO}_{2}$ to epoxides. Angew. Chem. Int. Ed. 49, 9822-9837 (2010).

7. Rintjema, J., Guo, W., Martin, E., Escudero-Adán, E. C. \& Kleij, A. W. Highly chemo-selective catalytic coupling of substituted oxetanes and carbon dioxide. Chem. Eur. J. 21, 10754-10762 (2015).

8. Yang, Z.-Z., Li, Y.-N., Wei, Y.-Y. \& He, L.-N. Protic onium salts-catalyzed synthesis of 5-aryl-2oxazolidinones from aziridines and $\mathrm{CO}_{2}$ under mild conditions. Green Chem. 13, 2351-2353 (2011). 
9. Whiteoak, C. J., Kielland, N., Laserna, V., Escudero-Adán, E. C., Martin, E. \& Kleij, A. W. A powerful aluminum catalyst for the synthesis of highly functional organic carbonates. J. Am. Chem. Soc. 135, 1228-1231 (2013).

10. Yu, B. \& He, L.-N. Upgrading carbon dioxide by incorporation into heterocycles. ChemSusChem 8, 5262 (2015).

11. Minakata, S., Sasaki, I. \& Ide, T. Atmospheric $\mathrm{CO}_{2}$ fixation by unsaturated alcohols using $t \mathrm{BuOl}$ under neutral conditions. Angew. Chem. Int. Ed. 49, 1309-1311 (2010).

12. Song, Q.-W., Zhou, Z.-H. \& He, L.-N. Efficient, selective and sustainable catalysis of carbon dioxide. Green. Chem. 19, 3707-3728 (2017).

13. Vara, B. A., Struble, T. J., Wang, W., Dobish, M. \& Johnston, J. N. Enantioselective small molecule synthesis by carbon dioxide fixation using a dual brønsted acid/base organocatalyst. J. Am. Chem. Soc. 137, 7302-7305 (2015).

14. Song Q.-W. \& He, L.-N. Robust silver(I) catalyst for the carboxylative cyclization of propargylic alcohols with carbon dioxide under ambient conditions. Adv. Synth. Catal. 358, 1251-1258 (2016).

15. Yoshida, S., Fukui, K., Kikuchi, S. \& Yamada, T. Silver-catalyzed enantioselective carbon dioxide incorporation into bispropargylic alcohols. J. Am. Chem. Soc. 132, 4072 (2010).

16. Martín, C., Fiorani, G. \& Kleij, A. W. Recent advances in the catalytic preparation of cyclic organic carbonates. ACS Catal. 5, 1353-1370 (2015).

17. Shaikh, R. R., Pornpraprom, S. \& D'Elia, V. Catalytic strategies for the cycloaddition of pure, diluted, and waste $\mathrm{CO}_{2}$ to epoxides under ambient conditions. ACS Catal. 8, 8, 419-450 (2018).

18. Comerford, J. W., Ingram, I. D. V., North, M. \& Wu, X. Sustainable metal-based catalysts for the synthesis of cyclic carbonates containing five-membered rings. Green Chem. 17, 1966-1987 (2015).

19. Ishida, N., Shimamoto, Y. \& Murakami, M. Solar-driven incorporation of carbon dioxide into a-amino ketones. Angew. Chem. Int. Ed. 51, 11750-11752 (2012).

20. Rintjema, J. \& Kleij, A. W. Substrate-assisted carbon dioxide activation as a versatile approach for heterocyclic synthesis. Synthesis 48, 3863-3878 (2016).

21. Aida, T. \& Inoue, S. Activation of carbon dioxide with aluminum porphyrin and reaction with epoxide. Studies on (tetraphenylporphinato)aluminum alkoxide having a long oxyalkylene chain as the alkoxide group. J. Am. Chem. Soc. 105, 1304-1309 (1983).

22. Kojima, F., Aida, T. \& Inoue, S. Fixation and activation of carbon dioxide on aluminum porphyrin. Catalytic formation of a carbamic ester from carbon dioxide, amine, and epoxide. J. Am. Chem. Soc. 108, 391-395 (1986).

23. Castro Osma, J. A., North, M., Offermans, W. K., Leitner, W. \& Müller, T. E. Unprecedented carbonato intermediates in cyclic carbonate synthesis catalysed by bimetallic aluminium(salen) complexes. ChemSusChem 9, 791-794 (2016). 
24. Rintjema, J., Epping, R., Fiorani, G., Martín, E., Escudero-Adán, E. C. \& Kleij, A. W. Substrate Controlled Product Divergence in $\mathrm{CO}_{2}$ Conversion to Heterocyclic Products. Angew. Chem. Int. Ed. 55, 3972-3976 (2016).

25. Laserna, V., Martin, E., Escudero-Adán, E. C. \& Kleij, A. W. Substrate-triggered stereoselective preparation of highly substituted organic carbonates. ACS Catal. 7, 5478-5482 (2017).

26. Dibenedetto, A., Aresta, M., Giannoccaro, P., Pastore, C., Pápai, I. \& Schubert, G. On the existence of the elusive monomethyl ester of carbonic acid $\left[\mathrm{CH}_{3} \mathrm{OC}(\mathrm{O}) \mathrm{OH}\right]$ at $300 \mathrm{~K}:{ }^{1} \mathrm{H}$ and ${ }^{13} \mathrm{C} N M R$ measurements and DFT calculations. Eur. J. Inorg. Chem. 2006, 908-913 (2006).

27. Heldebrant, D. J., Jessop, P. G., Thomas, C. A., Eckert, C. A. \& Liotta, C. L. The reaction of 1,8diazabicyclo[5.4.0]undec-7-ene (DBU) with carbon dioxide. J. Org. Chem. 70, 5335-5338 (2005).

28. West, K. N., Wheeler, C., McCarney, J. P., Griffith, K. N., Bush, D., Liotta, C. L. \& Eckert, C. A. In situ formation of alkylcarbonic acids with $\mathrm{CO}_{2}$. J. Phys. Chem. A 105, 3947-3948 (2001).

29. Gassensmith, J. J., Furukawa, H., Smaldone, R. A., Forgan, R. S., Botros, Y. Y., Yaghi, O. M. \& Fraser Stoddart, J. Strong and reversible binding of carbon dioxide in a green metal-organic framework. J. Am. Chem. Soc. 133, 15312-15315 (2011).

30. Jessop, P. G., Heldebrant, D. J., Li, X., Eckert, C. A. \& Liotta, C. L. Green chemistry: Reversible nonpolar-to-polar solvent. Nature 436, 1102-1102 (2005).

31. McDonald, T. M., Mason, J. A., Kong, X., Bloch, E. D., Gygi, D., Dani, A., Crocellà, V., Giordanino, F., Odoh, S. O., Drisdell, W. S., Vlaisavljevich, B., Dzubak, A. L., Poloni, R., Schnell, S. K., Planas, N., Lee, K., Pascal, T., Wan, L. F., Prendergast, D., Neaton, J. B., Smit, B., Kortright, J. B., Gagliardi, L., Bordiga, S., Reimer, J. A. \& Long, J. R. Cooperative insertion of $\mathrm{CO}_{2}$ in diamine-appended metalorganic frameworks. Nature 519, 303-308 (2015).

32. Whiteoak, C. J., Nova, A., Maseras, F. \& Kleij, A. W. Merging sustainability with organocatalysis in the formation of organic carbonates by using $\mathrm{CO}_{2}$ as a feedstock. ChemSusChem 5, 2032-2038 (2012).

33. Gennen, S., Alves, M., Méreau, R., Tassaing, T., Gilbert, B., Detrembleur, C., Jerome, C. \& Grignard, B. Fluorinated alcohols as activators for the solvent-free chemical fixation of carbon dioxide into epoxides. ChemSusChem 8, 1845-1849 (2015).

34. Sopeña, S., Martín, E., Escudero-Adán, E. C. \& Kleij, A. W. Pushing the limits with squaramide-based organocatalysts in cyclic carbonate synthesis. ACS Catal. 7, 3532-3539 (2017).

35. Toda, Y., Komiyama, Y., Kikuchi, A. \& Suga, H. Tetraarylphosphonium salt-catalyzed carbon dioxide fixation at atmospheric pressure for the synthesis of cyclic carbonates. ACS Catal. 6, 6906-6910 (2016).

36. Della Monica, F., Buonerba, A., Grassi, A., Capacchione, C. \& Milione, S. Glycidol: an hydroxylcontaining epoxide playing the double role of substrate and catalyst for $\mathrm{CO}_{2}$ cycloaddition reactions. ChemSusChem 9, 3457-3464 (2016).

37. Vilotijevic, I. \& Jamison, T. F. Epoxide-opening cascades promoted by water. Science 317, 1189-1192 (2007). 
38. Morten, C. J., Byers, J. A. \& Jamison, T. F. Evidence that epoxide-opening cascades promoted by water are stepwise and become faster and more selective after the first cyclization. J. Am. Chem. Soc. 133, 1902-1908 (2011).

39. Xia, Y. et al. An Unexpected Role of a Trace Amount of Water in Catalyzing Proton Transfer in Phosphine-Catalyzed $(3+2)$ Cycloaddition of Allenoates and Alkenes. J. Am. Chem. Soc. 129, 34703471, (2007).

40. Yu, J. S., Liu, Y. L., Tang, J., Wang, X. \& Zhou, J. Highly Efficient “On Water” Catalyst-Free Nucleophilic Addition Reactions Using Difluoroenoxysilanes: Dramatic Fluorine Effects. Angew. Chem. Int. Ed. 53, 9512-9516, (2014).

41. Shin, J. W., Hammer, N. I., Diken, E. G., Johnson, M. A., Walters, R. S., Jaeger, T. D., Duncan, M. A., Christie, R. A. \& Jordan, K. D. Infrared signature of structures associated with the $\mathrm{H}^{+}\left(\mathrm{H}_{2} \mathrm{O}\right) n(n=6$ to 27) clusters. Science 304, 1137-1140 (2004).

42. Miyazaki, M., Fuji, A., Ebata, T. \& Mikami, N. Infrared spectroscopic evidence for protonated water clusters forming nanoscale cages. Science 304, 1134-1137 (2004).

43. Garczarek, F. \& Gerwert, K. Functional waters in intraprotein proton transfer monitored by FTIR difference spectroscopy. Nature 439, 109-112 (2006).

44. Seo, J., Warnke, S., Pagel, K., Bowers, M. T. \& von Helden, G. Infrared spectrum and structure of the homochiral serine octamer-dichloride complex. Nat. Chem. 9, 1263-1268 (2017).

45. Baker, M. J., Trevisan, J., Bassan, P., Bhargava, R., Butler. H. J., Dorling, K. M., Fielden, P. R., Fogarty, S. W., Fullwood, N. J., Heys, K. A., Hughes, C., Lasch, P., Martin-Hirsch, P. L., Obinaju, B., Sockalingum, G. D., Sulé-Suso, J., Strong, R. J., Walsh, M. J., Wood, B. R., Gardner, P. \& Martin, F. L. Using fourier transform IR spectroscopy to analyze biological materials. Nat. Protoc. 9, 1771-1791 (2014).

46. Adato, R. \& Altug, H. In-situ ultra-sensitive infrared absorption spectroscopy of biomolecule interactions in real time with plasmonic nanoantennas. Nat. Commun. 4, 2154 (2013).

47. Yang, G. \& Xu, Y. Probing chiral solute-water hydrogen bonding networks by chirality transfer effects: A vibrational circular dichroism study of glycidol in water. J. Chem. Phys. 130, 164506 (2009).

48. Conrad, A. R., Teumelsan, N. H., Wang, P. E. \& Tubergen, M. J. A spectroscopic and computational investigation of the conformational structural changes induced by hydrogen bonding networks in the glycidol-water complex. J. Phys. Chem. A 114, 336-342 (2010).

49. Cano, I., Chapman, A. M., Urakawa, A. \& van Leeuwen, P. W. N. M. Air-stable gold nanoparticles ligated by secondary phosphine oxides for the chemoselective hydrogenation of aldehydes: crucial role of the ligand. J. Am. Chem. Soc. 136, 2520-2528 (2014).

50. Urakawa, A., Jutz, F., Laurenczy, G. \& Baiker, A. Carbon dioxide hydrogenation catalyzed by a ruthenium dihydride: a DFT and high-pressure spectroscopic investigation. Chem. Eur. J. 13, 38863899 (2007). 
51. Ema, T., Fukuhara, K., Sakai, T., Ohbo, M., Bai, F.-Q. \& Hasegawa, J.-Y. Quaternary ammonium hydroxide as a metal-free and halogen-free catalyst for the synthesis of cyclic carbonates from epoxides and carbon dioxide. Catal. Sci. Technol. 5, 2314-2321 (2015).

52. Whiteoak, C. J., Kielland, N., Laserna, V., Castro-Gómez, F., Martin, E., Escudero-Adán, E. C., Bo, C. \& Kleij, A. W. Highly active aluminium catalysts for the formation of organic carbonates from $\mathrm{CO}_{2}$ and oxiranes. Chem. Eur. J. 20, 2264-2275 (2014).

53. North, M. \& Pasquale, R. Mechanism of cyclic carbonate synthesis from epoxides and $\mathrm{CO}_{2}$. Angew. Chem. Int. Ed. 48, 2946-2948 (2009).

54. Darensbourg, D. J. \& Yarbrough, J. C. Mechanistic aspects of the copolymerization reaction of carbon dioxide and epoxides using a chiral salen chromium chloride catalyst. J. Am. Chem. Soc. 124, 63356342 (2002).

55. Darensbourg, D. J., Yarbrough, J. C., Ortiz, C. \& Fang, C. C. Comparative kinetic studies of the copolymerization of cyclohexene oxide and propylene oxide with carbon dioxide in the presence of chromium salen derivatives. In situ FTIR measurements of copolymer vs cyclic carbonate production. J. Am. Chem. Soc. 125, 7586-7591 (2003).

56. Garand, E., Wende, T., Goebbert, D. J., Bergmann, R., Meijer, G., Neumark, D. M. \& Asmis, K. R. Infrared spectroscopy of hydrated bicarbonate anion clusters: $\mathrm{HCO}_{3}{ }^{-}\left(\mathrm{H}_{2} \mathrm{O}\right)_{1-10}$. J. Am. Chem. Soc. 132, 849-856 (2010).

57. Álvarez-Moreno, M.; de Graaf, C.; Lopez, N.; Maseras, F.; Poblet, J.M.; Bo, C. Managing the Computational Chemistry Big Data Problem: The ioChem-BD Platform J. Chem. Inf. Model., 55, 95-103 (2015).

\section{Acknowledgments}

The authors acknowledge financial support by ICIQ, ICREA, the CERCA Program/Generalitat de Catalunya and the Spanish Ministerio de Economı y Competitividad (MINECO: CTQ2012-34153, CTQ-2014-60419R, CTQ2016-75499-R (AEI/FEDER-UE), and Severo Ochoa Excellence Accreditation 2014-2018, SEV2013-0319). R.H. thanks the COFUND postdoctoral program of the E.U. The Research Support Area of $\mathrm{ICIQ}$ is also thanked for their experimental assistance.

\section{Author contributions}

A. W. K. and A. U. conceived of the project. R. H. and J. R. (equal contribution) respectively carried out the spectroscopic measurements and the catalytic experiments, as well as the manuscript preparation. J. G. F. and C. B. performed DFT calculations. E. M. and E. C. E. helped X-ray analysis. All authors contributed to scientific discussion and revised the manuscript.

\section{Competing interests}

The authors declare no competing financial interests.

\section{Additional information}


Supplementary information is available for this paper at https://doi.org/xxxxx.

Reprints and permissions information is available at www.nature.com/reprints.

Correspondence and requests for materials should be addressed to C.B., A.U., and A.W.K.

Publisher's note: Springer Nature remains neutral with regard to jurisdictional claims in published maps and institutional affiliations. 


\section{Supporting information for:}

\section{Caught in the act: Deciphering key intermediates in the transformation of carbon dioxide into heterocyclic products}

Rui Huang, ${ }^{1 \#}$ Jeroen Rintjema, ${ }^{1 \#}$ Joan González-Fabra, ${ }^{1}$ Eddy Martín, ${ }^{1}$ Eduardo C. Escudero-
Adán, ${ }^{1}$ Carles Bo, ${ }^{1 *}$ Atsushi Urakawa ${ }^{*}$ and Arjan W. Kleij ${ }^{1,2 *}$

${ }^{1}$ Institute of Chemical Research of Catalonia (ICIQ), the Barcelona Institute of Science and Technology, Av. Països Catalans 16, 43007 Tarragona, Spain, E-mail: akleij@iciq.es: ${ }^{2}$ Catalan Institute of Research and Advanced Studies (ICREA), Pg. Lluís Companys 23, 08010 Barcelona, Spain.

\#Equal contribution, *Correspondence to cbo@iciq.cat, aurakawa@iciq.es and akleij@iciq.es.

\section{Contents:}

Page S2: Catalyst synthesis and general conditions

Page S3: $\quad$ Typical catalytic experiments

Page S5: D-labeling

Page S6: Chiral substrate conversions

Page S7: DFT calculations

Page S8: $\quad$ Computational details

Page S9: $\quad$ Reaction order in catalyst

Page S10: Regioselectivity of carbon dioxide insertion

Page S11: Enantioselectivity and racemization of the product

Page S13: DFT functionals

Page S14: Vibrational frequencies analysis: DFT and experiments

Page S15: Kinetic studies

Page S16: Role of water

Page S17: In situ ATR-IR spectroscopy in solution

Page S18: Operando HPIR measurements

Page S19: Interactions of $\mathrm{Al}(\mathrm{L})$ with O-containing reagents

Page S20: $\quad X$-ray structure for $\mathrm{Al}^{\mathrm{GLY}} \mathrm{L}$

Page S22: Hydrogen bonding between glycidol and water

Page S23: $\quad \mathrm{CO}_{2}$ trapping measurements from other substrates

Page S24: Fingerprint regions of in-situ ATR-IR spectra

Page S25: $\quad$ Ligand assisted substrate activation

Page S26: Peak-fitting of operando HP-IR spectra

Page S29: $\quad$ NMR spectra

Page S36: References

\section{Catalytic experiments}

\section{Catalyst synthesis and general conditions:}

Both the ligands ${ }^{[1]}$ and catalysts ${ }^{[2]}$ (see Figure $S 1$ below) were synthesized according to previously reported procedures. The modified synthesis procedure of the $\mathrm{Al}(\mathrm{L})$ complex is indicated in Figure $\mathrm{S} 1 .{ }^{1} \mathrm{H}$ and ${ }^{13} \mathrm{C}$ NMR spectra were recorded on a Bruker 
AV-300, AV-400 or AV-500 spectrometer. Mass spectrometric analysis and X-ray diffraction studies were performed by the Research Support Group at the ICIQ. Carbon dioxide was purchased from PRAXAIR and used without further purification. Solvents used in the synthesis of the complexes were dried using an Innovative Technology PURE SOLV solvent purification system.

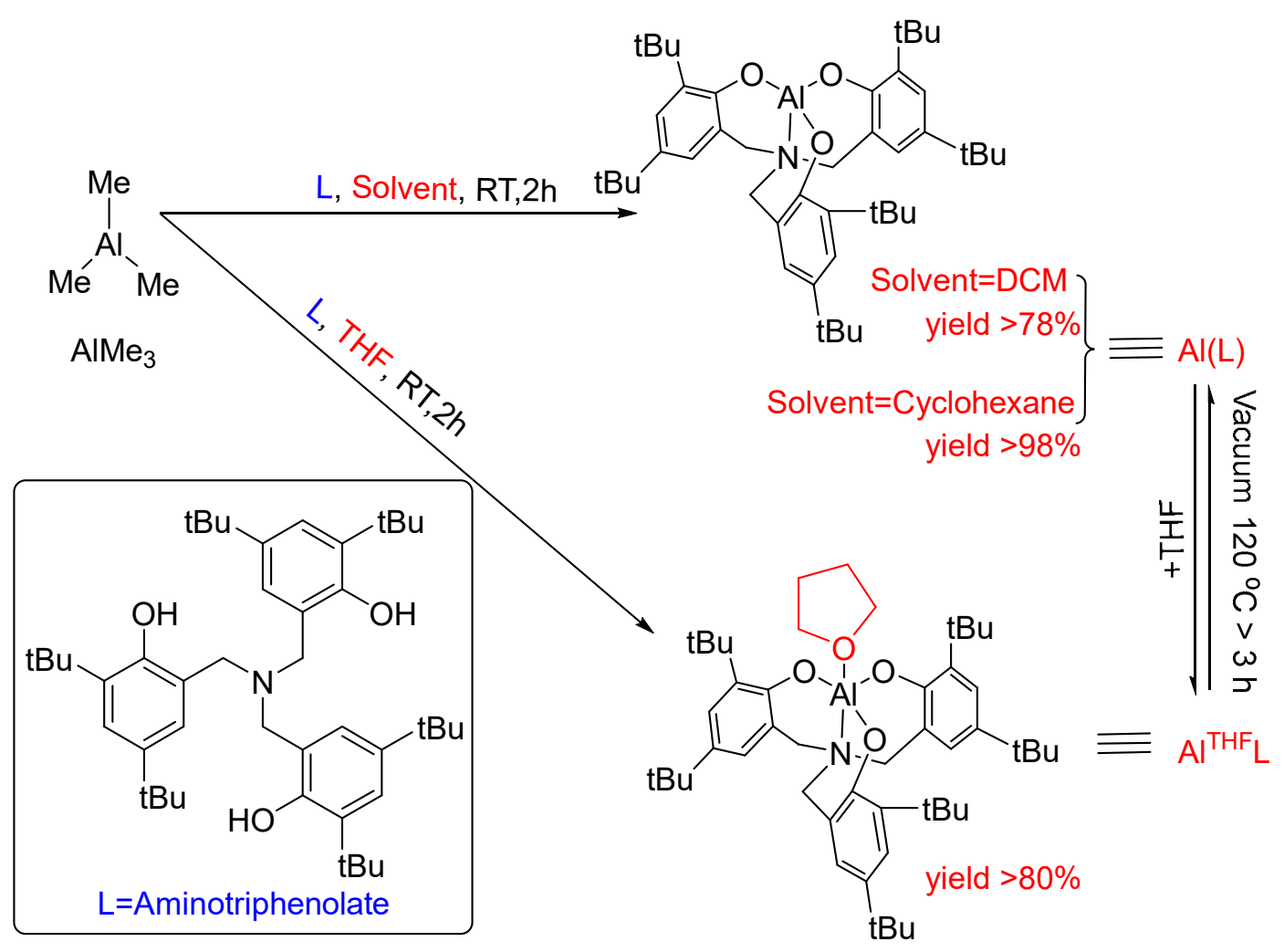

Figure S1. Al-complex synthesis 


\section{Typical catalytic experiment}

The respective epoxide, Al-complex, internal standard and solvent were charged into a $30 \mathrm{~mL}$ stainless steel autoclave. The autoclave was then subjected to three cycles of pressurization and depressurization with carbon dioxide (5 bar), before final stabilization of the pressure to 10 bar. The autoclave was sealed and heated to the required temperature and left stirring. At the end of the reaction an aliquot of the resulting mixture was taken and the conversion was determined by means of ${ }^{1} \mathrm{H}$ NMR spectroscopy using $\mathrm{CDCl}_{3}$ as the solvent. The identities of the cyclic carbonate products were confirmed by comparison to literature data.

Table S1 Comparison of reactivity among different catalysts

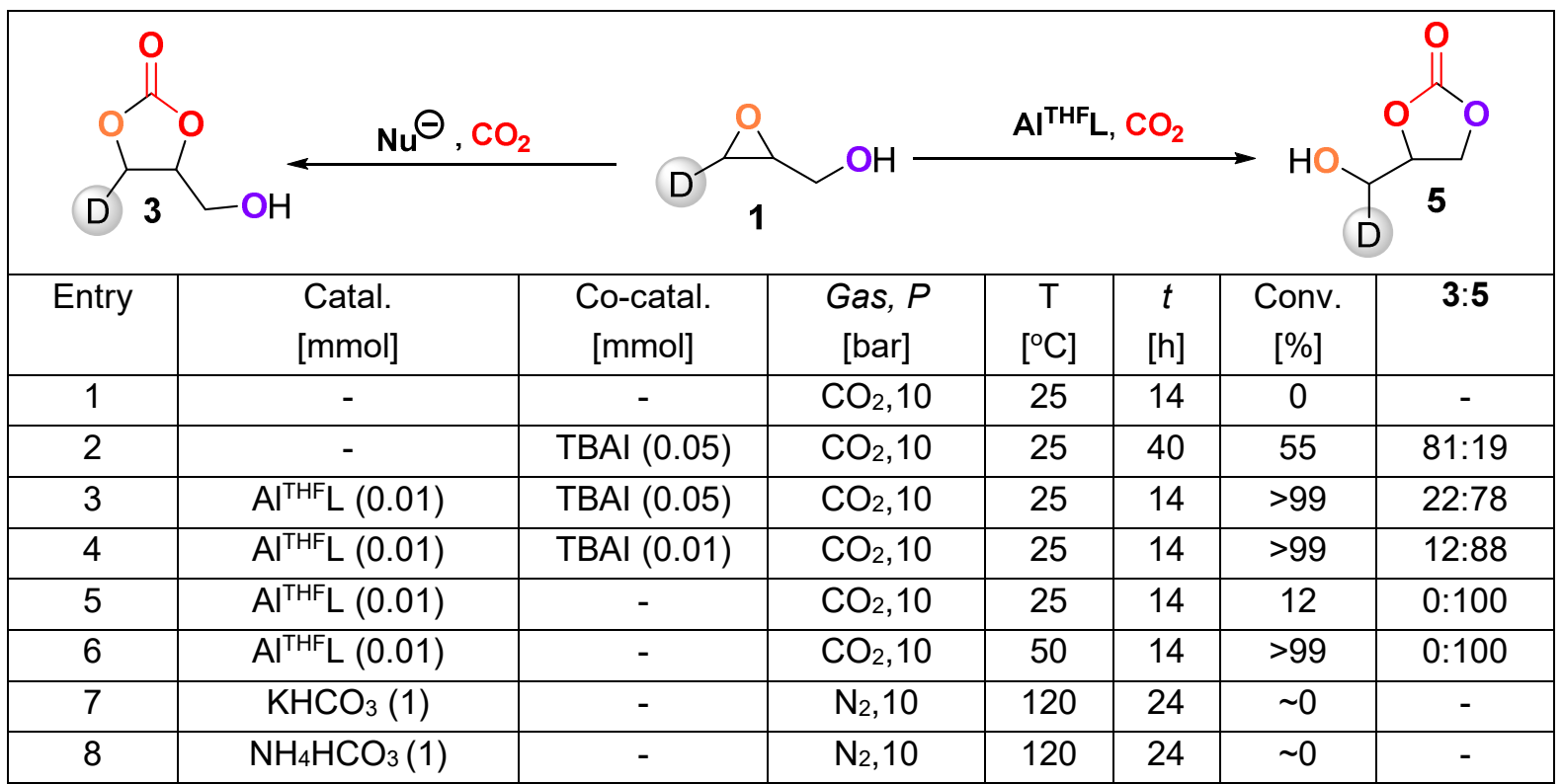

Reaction conditions: $1.0 \mathrm{mmol}$ of substrate (glycidol) and $1.0 \mathrm{~mL}$ of solvent (2-butanone) were used in each reaction; the yield was determined by ${ }^{1} \mathrm{H} \mathrm{NMR}\left(\mathrm{CDCl}_{3}\right)$, and mesitylene was used as internal standard. TBAI= tetrabutylammonium iodide.

Note that a classical double inversion route that involves epoxide coordination to the Al-complex followed by nucleophilic ring opening by iodide is to some extent competitive (cf., formation of product 3 ) in the presence of the Al complex (entries 3 and 4). However, when only the Al complex is present (entries 5 and 6), only product 5 is formed in line with the hydroxyl activation of Figure 1 in the main text. 
Table S2. Potential triol formation by hydrolysis

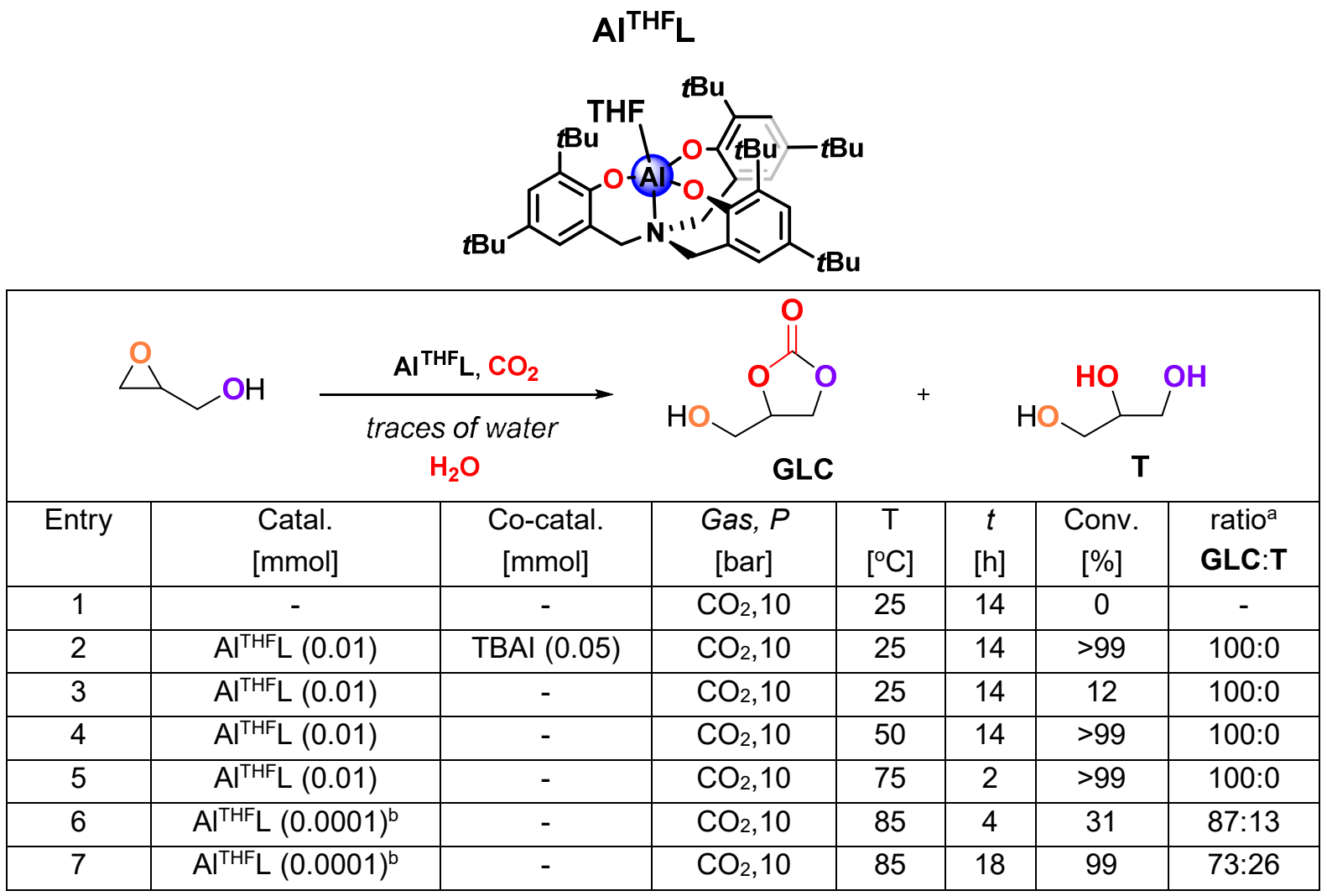

Reaction conditions: $1.0 \mathrm{mmol}$ of substrate (glycidol) and $1.0 \mathrm{~mL}$ of solvent (2-butanone) were used in each reaction; the yield was determined by ${ }^{1} \mathrm{H} N M R\left(\mathrm{CDCl}_{3}\right)$, and mesitylene was used as internal

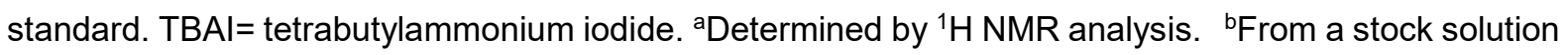
prepared in 2-butanone (MEK).

From the data presented in Table S2, it is clear that glycerol (triol) formation only occurs when the catalyst $\mathbf{A} \mathbf{I}^{\mathrm{THF}} \mathbf{L}$ is present in very low amounts. In the experiments carried out in the main text, the amount of $\mathbf{A} \mathbf{I}^{\mathrm{THF}} \mathbf{L}$ is much higher and therefore it is not likely that triol formation takes place. 


\section{D-labeling}

Deuterium labeled glycidol was synthesized according to the procedure shown below in Figure S2 and Figure S3. Key step is the formation of compound $\mathbf{C}$, which was prepared according to a previously published procedure. ${ }^{[3]}$ Spectra of the compounds C-F show around $15 \%$ of their non-deuterated analogues.

\section{Synthesis of D-labeled glycidol}
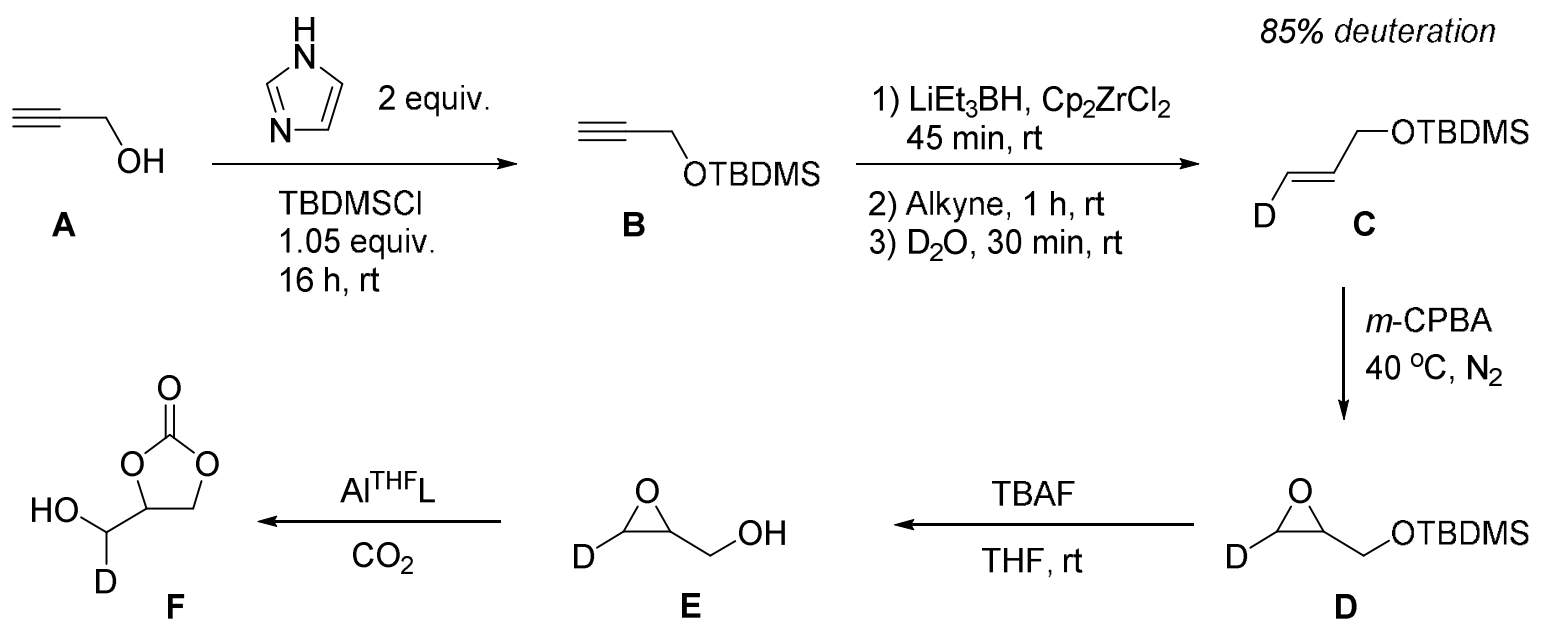

Figure S2. Synthesis of D-labeled glycidol starting from propargylic alcohol A. 


\section{Chiral Substrate Conversions}

\section{Deuterium labeled glycidol as a model system for the racemization}

a)<smiles>[2H]C1OC1CO</smiles>

c)<smiles>[2H]C(O)C1COC(=O)O1</smiles>

b)<smiles>[2H]C1OC1CO</smiles><smiles>[2H]C(O)C1COC(=O)O1</smiles><smiles>[2H]C1OC(=O)OC1CO</smiles>

Deuterium labeled glycidol as a model system for the racemization<smiles>O=C(O)COC[C@H]1CO1</smiles>

(S)-GLY<smiles>O=C1OC[C@@H](CO)O1</smiles>

(R)-GLC
N, N-dimethyl benzylamine<smiles>O=C1OC[C@@H](CO)O1</smiles>

(rac)-GLC

Figure S3. Top panel: Control experiments using deuterium labeling show that no substrate conversion or product racemization occurs under the catalytic conditions. Bottom panel: the conversion of chiral glycidol (S)-GLY into (rac)-GLC. 


\section{DFT calculations}

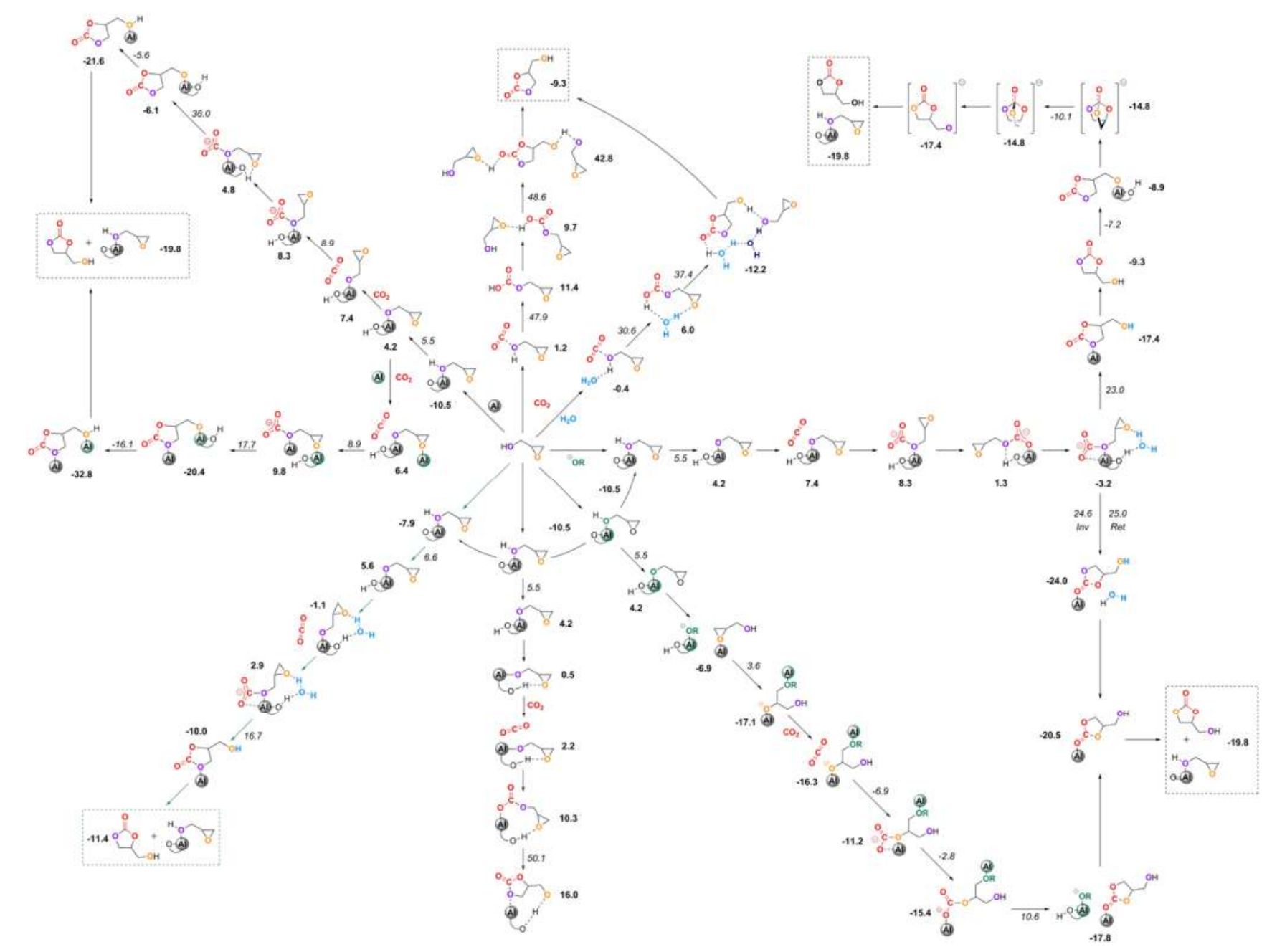

Figure S4. Schematic representation of all possible mechanistic routes to GLC explored with DFT methods. Gibbs energy values $\left(\mathrm{kcal} \cdot \mathrm{mol}^{-1}\right)$ for intermediates are indicated in bold and for transition states in italic. A PDF file is available at https://figshare.com/s/81493e312482f1a2bb77. 


\section{Computational details}

All calculations in this study were carried out by using Gaussian 09 package. ${ }^{[4]}$ Two kinds of dispersion-corrected DFT functionals were used to optimize geometries and evaluate energies: the $\omega \mathrm{B} 97 \times \mathrm{DD}^{[5]}$ and the B97-D3.[6] It is worth to note both functionals give similar results in most of the cases although for some weakly bound structures the different treatment of dispersion effects makes a difference. The standard $6-311 \mathrm{G}(\mathrm{d}, \mathrm{p})$ basis set ${ }^{[7]}$ was used to describe all atoms. Full geometry optimizations were performed without any constrain. The nature of the encountered stationary points was characterized either as minima or transition states by means of harmonic vibrational frequencies analysis. Gibbs free energies were calculated at experimental conditions $(T=323.15 \mathrm{~K}, \mathrm{P}=1$ atm). Entropic corrections were included for all calculations in order to model the translational entropy in solvation. [8] For the sake of comparison with experimentally measured infrared spectra, several DFT functionals were used: $\omega \mathrm{B} 97 \times \mathrm{D}$ with scaling factor $0.957,,^{[9]} \mathrm{B} 3 \mathrm{PW} 91^{[10]}$ with scaling factor $0.963,{ }^{[9]}$ and BP86 [11] unscaled.

Solvent effects were accounted for in all calculations by using the Solvation Model based on Density (SMD) as implemented in Gaussian. The dielectric constant $(\varepsilon)$ of the polarizable medium was set to the value reported for butanone, which is the solvent used in the experiments $(\varepsilon=18.246)$. ${ }^{[12]}$

A data set collection of computational results is available in the ioChem-BD repository ${ }^{[13]}$ and can be accessed via http://dx.doi.org/10.19061/iochem-bd-1$\underline{58}$. 


\section{Reaction order in catalyst}

The reaction order in catalyst was one of the key unknowns of the GLC mechanism. The presence of two oxygen atoms in GLY may have two Alcomplexes involved in the initial substrate activation process by first deprotonating the alcohol unit, followed by $\mathrm{CO}_{2}$ insertion and epoxide ringopening to obtain the cyclic carbonate. The results presented in Figure S5 show that coordination of the epoxide oxygen (orange) to a second Al-complex stabilizes by $18.3 \mathrm{kcal} \cdot \mathrm{mol}^{-1}$ the TS-CC related to the ring-opening of the epoxide by the carbonate group (red). The intermediate CC is also more stable when attached at two Al-complexes, which indicates a more favored route than in the monometallic mechanism. It may therefore be anticipated that the bimetallic mechanism should be the preferred route to obtain GLC but the stabilization of the alkoxide species that is generated after the epoxide ring opening turned out to a crucial feature. Water, as will be demonstrated, can also play this stabilization role with the possibility of acting as a proton shuttle.

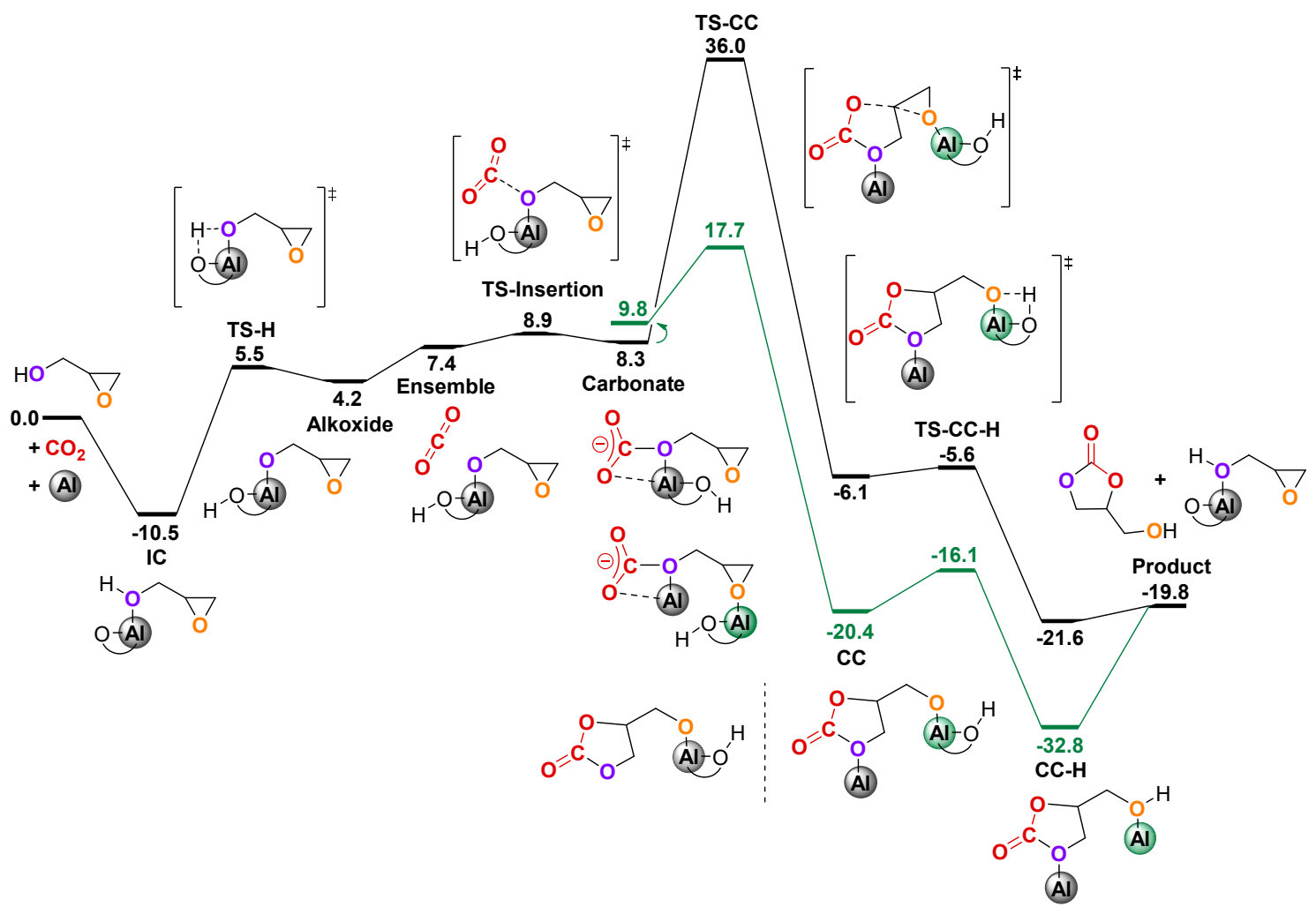

Figure S5. Gibbs energy profile $\left(\mathrm{kcal} \cdot \mathrm{mol}^{-1}\right)$ of the monometallic (black line) and bimetallic (green line) mechanisms for GLC formation. 


\section{Regioselectivity of the carbon dioxide insertion}

Carbon dioxide could be activated by either of the two oxygen atoms present in GLY, namely OEp (oxygen atom of the epoxide) and Oal (oxygen atom of the alcohol). The results depicted in Figure $\mathbf{S 6}$ show that there is no significant preference between the two reactive sites, since OEp insertion presents an absolute barrier of $27.8 \mathrm{kcal} \cdot \mathrm{mol}^{-1}$ and insertion of $\mathrm{CO}_{2}$ to $\mathrm{OH}$ is slightly more energetically demanding by $0.4 \mathrm{kcal} \cdot \mathrm{mol}^{-1}$.

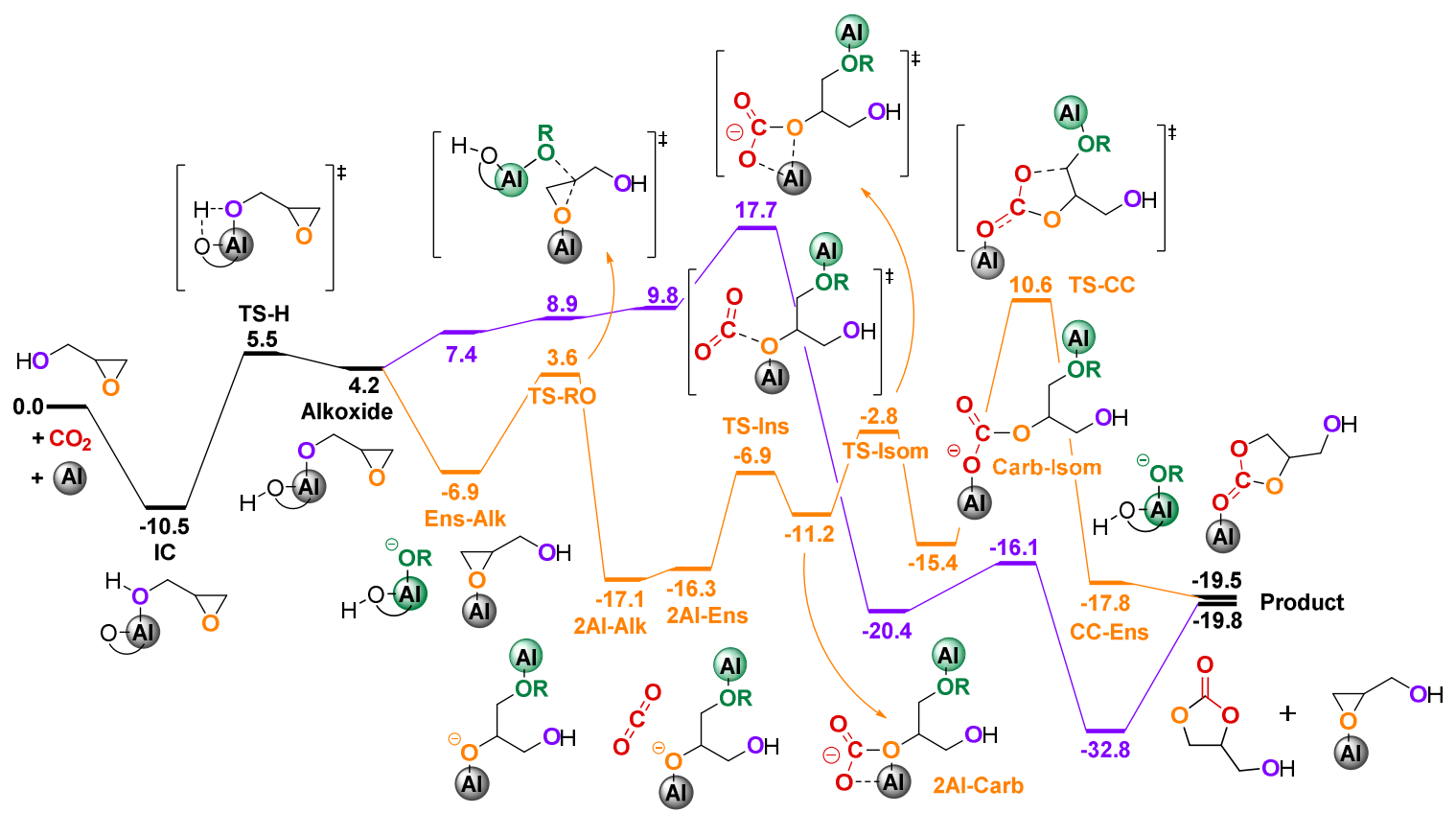

Figure S6. Gibbs energy profile $\left(\mathrm{kcal} \cdot \mathrm{mol}^{-1}\right)$ of the bimetallic mechanisms for the $\mathrm{CO}_{2}$ insertion to the Oal alcohol oxygen (purple line) and Oep epoxide oxygen (orange line). 


\section{Enantioselectivity and racemization of the product}

The enantio-selectivity of the process has been studied computationally to shed light on the enantiomeric excess obtained experimentally (Figure S7). The ringopening of the epoxide by the nucleophilic attack of the carbonate (TS-CC) is the reaction step that determines the enantio-selectivity. An $\mathrm{S}_{\mathrm{N}} 1$ type mechanism leads to racemization and $\mathrm{S}_{\mathrm{N}} 2$ type mechanism to inversion of the configuration. The computational results show a small difference between $\mathrm{S}_{\mathrm{N}} 2$ (black line) and $\mathrm{S}_{N} 1$ (green-blue lines) absolute barriers, which agrees with the experimental enantiomeric excess. However, the experimental conditions are too mild to overcome the high absolute barriers of both processes. Consequently, the enantiomeric excess cannot be explained by these results.

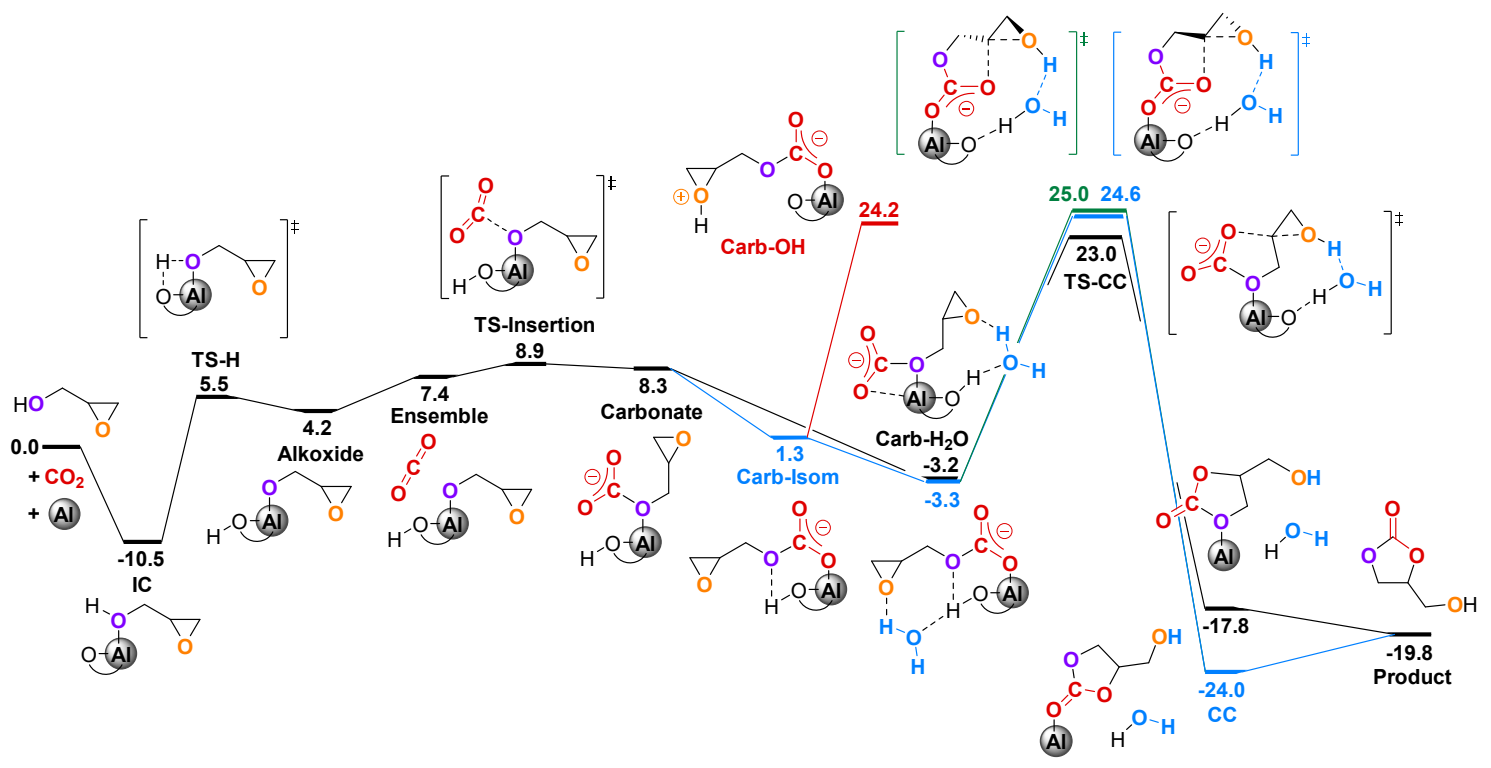

Figure S7. Gibbs energy profile $\left(\mathrm{kcal} \cdot \mathrm{mol}^{-1}\right)$ of the $\mathrm{S}_{\mathrm{N}} 1$ (blue-green line) and $\mathrm{S}_{\mathrm{N}} 2$ (black line) mechanisms for the GLC formation co-catalyzed by water.

The characterization of the product to determine the enantio-selectivity goes through an amination process to produce a chiral amide. The amine used to open the cyclic carbonate can also deprotonate the alcohol group present in the product through TS-CC-H (12.8 $\mathrm{kcal} \cdot \mathrm{mol}^{-1}$ for the green line) depicted in Figure S8. After the alkoxide is obtained (Alk-CC), it can rapidly proceed through TSRac to a stable symmetric intermediate (Int-Rac). Due to this symmetry, the TSRac leads to the final Product, which could either invert or retain the configuration. This process therefore leads to overall racemization. 


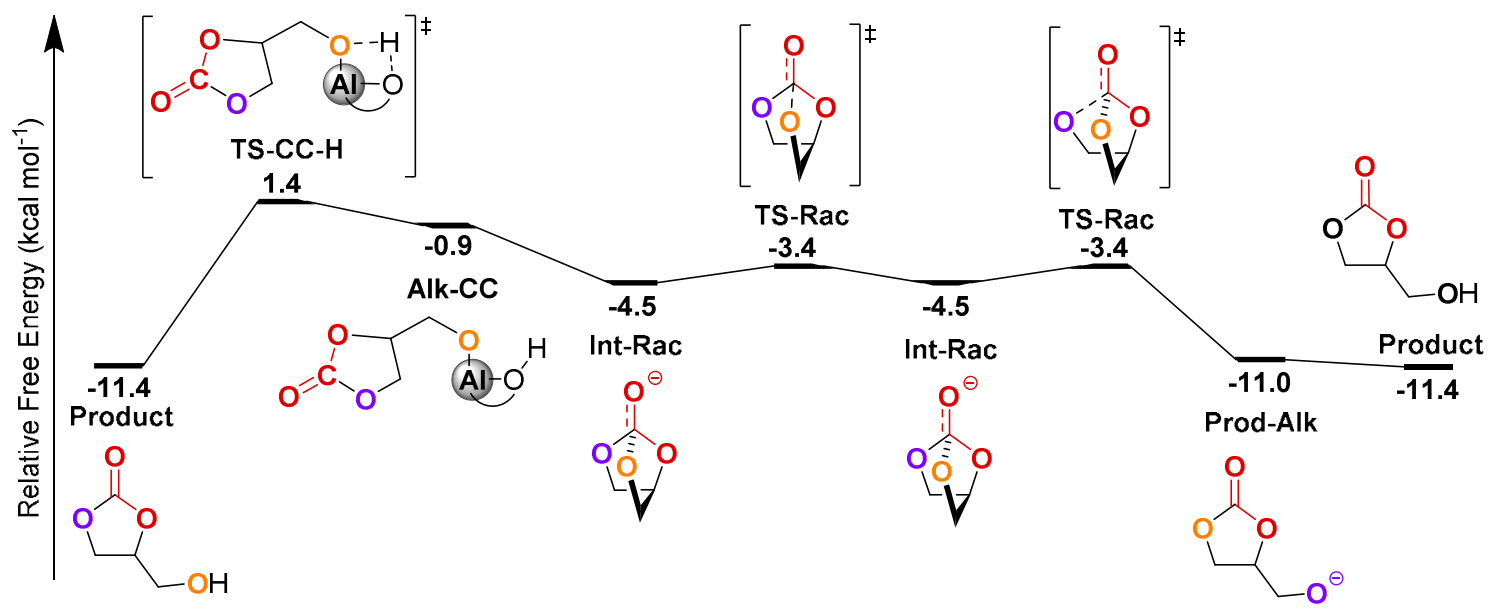

Figure S8. Gibbs energy profile of the acid-base racemization mechanism computed with B97-D3 functional. Note that the relative "zero" level of the energy scale in this Figure corresponds to the zero level in Figure 3. 


\section{DFT functionals}

We analyzed the performance of a diversity of DFT-based methods to describe the reaction mechanism. In Table S3 we have collected key geometric values for the characterization and description of relevant transition state, namely TS-CC$\mathrm{H}_{2} \mathrm{O}$ in Figure 3, which is the rate-determining transition state of the proposed mechanism. Angles and bond distances collected in Table $\mathbf{S} 3$ are representative of the geometry coordinates involved in the mentioned TS, which are (1) the O$\mathrm{C}-\mathrm{O}$ angle in $\mathrm{CO}_{2}$, (2) the distance between the carbon center of $\mathrm{CO}_{2}$ and the oxygen atom of the alkoxide moiety, (3) the distance between the carbon atom of the epoxide group and the approaching oxygen of $\mathrm{CO}_{2}$ and finally, (4) the C-O-C angle of the epoxide group. In summary, subtle differences in the main geometric parameters obtained at different levels reveal the different effect of empirical dispersion effects and the amount of HF exchange included. Although the differences are rather small, the mechanism description arising from the different methods is different, and this suggested the existence of two mechanism types. We observe that $\mathrm{C}_{\mathrm{CO}}-\mathrm{O} O \mathrm{OH}$ shorter bond distance and larger $\mathrm{CO}_{2}$ angle lead to a mechanism type we labeled as concerted. The larger the $\mathrm{CO}_{2}$ angle in the TS, the less carbonate character it has. Contrarily, smaller epoxide and $\mathrm{CO}_{2}$ angles prompt to what we called a step-wise mechanism, only observed for M06-2X and wB97xD functionals. This mechanism type is similar to the one proposed for the reaction without water. A double-hybrid DFT functional (B2PLYP), which includes both exact HF exchange and MP2 correlation corrections, is in line with most the other methods tested. In summary, most of the methods tested point to a concerted mechanism.

Table S3. Selected geometric parameters for $\mathbf{T S}-\mathbf{C C}-\mathrm{H}_{2} \mathrm{O}$ (ep: epoxide), free energy barrier (in $\mathrm{kcal} \cdot \mathrm{mol}^{-1}$ ), and mechanism type at different DFT levels.

\begin{tabular}{lcccccc}
\hline DFT & Angle $\mathrm{CO}_{2}$ & $\mathrm{~d}\left(\mathrm{C}_{\mathrm{CO} 2-} \mathrm{OoH}\right)$ & $\mathrm{d}\left(\mathrm{O}_{\mathrm{co} 2-} \mathrm{C}_{\mathrm{ep}}\right)$ & $\begin{array}{c}\text { Angle } \\
\text { epoxide }\end{array}$ & $\Delta \mathrm{G}^{\ddagger}$ & Mechanism \\
\hline B97D3-(D3BJ) & $\mathbf{1 5 0}$ & $\mathbf{1 . 9}$ & $\mathbf{2 . 2 5}$ & $\mathbf{9 0}$ & $\mathbf{2 4 . 7}$ & Concerted \\
B97D3-(D3) & 151 & 2 & 2.2 & 91 & 25.3 & Concerted \\
B3LYP-D3BJ & 139 & 1.6 & 2.4 & 80 & 26.2 & Concerted \\
B3LYP-D3 & 140 & 1.6 & 2.4 & 80 & 27.5 & Concerted \\
PBE & 142 & 1.7 & 2.3 & 78 & 31.1 & Concerted \\
BP86 & 145 & 1.8 & 2.3 & 81 & 33.7 & Concerted \\
BP86-D3 & 140 & 1.7 & 2.3 & 80 & 18.3 & Concerted \\
M06 & 160 & 2.2 & 1.9 & 101 & 31.5 & Concerted \\
M06-2X & 136 & 1.5 & 2.1 & 76 & 32.0 & Stepwise \\
wB97xD & 136 & 1.5 & 2.3 & 74 & 33.5 & Stepwise \\
B2PLYP-D3BJ & 139 & 1.6 & 1.9 & 79 & 28.0 & Concerted \\
\hline
\end{tabular}

Vibrational Frequency Analysis: DFT vs Experiments 
Aimed at identifying species responsible of the infrared signal experimentally observed at $1837 \mathrm{~cm}^{-1}$, we considered several candidates and computed the harmonic vibrational frequencies using some DFT methods. $\mathrm{CO}_{2}$ interacts with the glycidol alkoxide as well as with the aluminum metal center, forming a stable intermediate with a relative Gibbs energy of $-3.2 \mathrm{kcal} \cdot \mathrm{mol}^{-1}$. This species is the one that shows a vibration corresponding to the carbonate $\mathrm{C}=\mathrm{O}$ stretching in closest agreement with the experimental value.

Table S4. DFT-computed structures, carbonate $\mathrm{C}=\mathrm{O}$ vibrational frequency, and Gibbs energy. Experimental observed structures are highlighted in bold. Calculated vibrational frequency for B3PW91 and $\omega \mathrm{B} 97 \times \mathrm{D}$ are scaled by 0.963 and 0.957 , respectively.

\begin{tabular}{|c|c|c|c|c|c|c|c|c|}
\hline Structures & 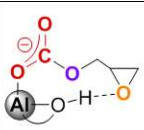 & 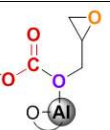 & 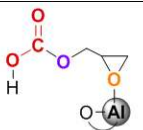 & 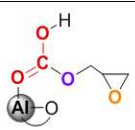 & (A1) & 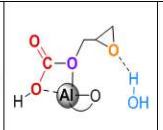 & $\mathrm{C}^{-\mathrm{H}}$ & $\mathrm{HO}$ \\
\hline BP86-Freq $\left(\mathrm{cm}^{-1}\right)$ & 1779 & 1775 & 1783 & 1687 & 1835 & 1758 & 1770 & 1805 \\
\hline B3PW91-Freq $\left(\mathrm{cm}^{-1}\right)$ & 1770 & 1782 & 1792 & 1698 & 1825 & 1765 & 1778 & 1812 \\
\hline & 1777 & 1796 & 1805 & 1698 & 1830 & 1795 & 1790 & 1825 \\
\hline Experimental $\left(\mathrm{cm}^{-1}\right)$ & & & & 1837 & & & & 1790 \\
\hline$\Delta \mathrm{G}\left(\mathrm{kcal} \mathrm{mol}^{-1}\right)$ & 1.3 & 8.1 & 2.2 & 3.0 & -3.2 & 4.6 & 12.0 & -9.3 \\
\hline
\end{tabular}




\section{Kinetic studies}

The reaction setup for the kinetic experiments differs slightly from the general catalytic procedure described on page S3. After loading all the reaction components into the reactor vessel, the reactor was first heated to $50{ }^{\circ} \mathrm{C}$ and afterwards pressurized with $\mathrm{CO}_{2}$. A reaction time of $2 \mathrm{~h}$ starts after this pressurization. The activation energy of the reaction was determined from the Eyring plot and the Arrhenius plot. Using the Arrhenius equation $\mathrm{k}=\mathrm{Ae} \mathrm{e}^{-\mathrm{Ea} / \mathrm{RT}}$ to plot the natural logarithm versus $1 / \mathrm{T}$, a straight line was obtained of which the slope is related to the activation energy.

For Eyring plot (Figure S9C):

$$
\begin{gathered}
\text { Slope }=-\Delta \mathrm{H} \ddagger / \mathrm{R} \text { and } \mathrm{y} \text {-intercept }=\Delta \mathrm{S} \ddagger / \mathrm{R}+\ln (\mathrm{kB} / \mathrm{h}) \\
\Delta \mathrm{H} \ddagger(\text { the enthalpy of activation })=22.7 \mathrm{kcal} \cdot \mathrm{mol}^{-1} \\
\Delta \mathrm{S} \ddagger(\text { the entropy of activation })=-64 \mathrm{~J} \cdot \mathrm{K}-1 \\
\mathrm{Ea}\left(50^{\circ} \mathrm{C}\right)=\Delta \mathrm{H} \ddagger+\mathrm{RT}=23.3 \mathrm{kcal} \cdot \mathrm{mol}-1
\end{gathered}
$$

For the Arrhenius plot (Figure S9D):

$$
\text { Slope }=- \text { Ea } / \mathrm{R}
$$

$$
\mathrm{Ea}=-\mathrm{R} \text { * slope }=-(1.987 \text { * } 10-3){ }^{*}-11738=23.3 \mathrm{kcal} \cdot \mathrm{mol}-1
$$

A \begin{tabular}{ccccc}
\cline { 2 - 6 } $\mathrm{T}(\mathrm{K})$ & $1000 / \mathrm{T}$ & $\mathrm{k}$ & $\mathrm{Ln} \mathrm{k}$ & $\mathrm{Ln}(\mathrm{k} / \mathrm{T})$ \\
\hline 298 & 3.36 & $2.54 \mathrm{E}-06$ & -12.88 & -18.58 \\
308 & 3.25 & $1.45 \mathrm{E}-05$ & -11.14 & -16.87 \\
313 & 3.19 & $2.09 \mathrm{E}-05$ & -10.78 & -16.52 \\
323 & 3.10 & $4.38 \mathrm{E}-05$ & -10.04 & -15.81 \\
328 & 3.05 & $7.99 \mathrm{E}-05$ & -9.43 & -15.23 \\
338 & 2.96 & $3.63 \mathrm{E}-04$ & -7.92 & -13.74 \\
348 & 2.87 & $8.94 \mathrm{E}-04$ & -7.02 & -12.87 \\
\hline
\end{tabular}
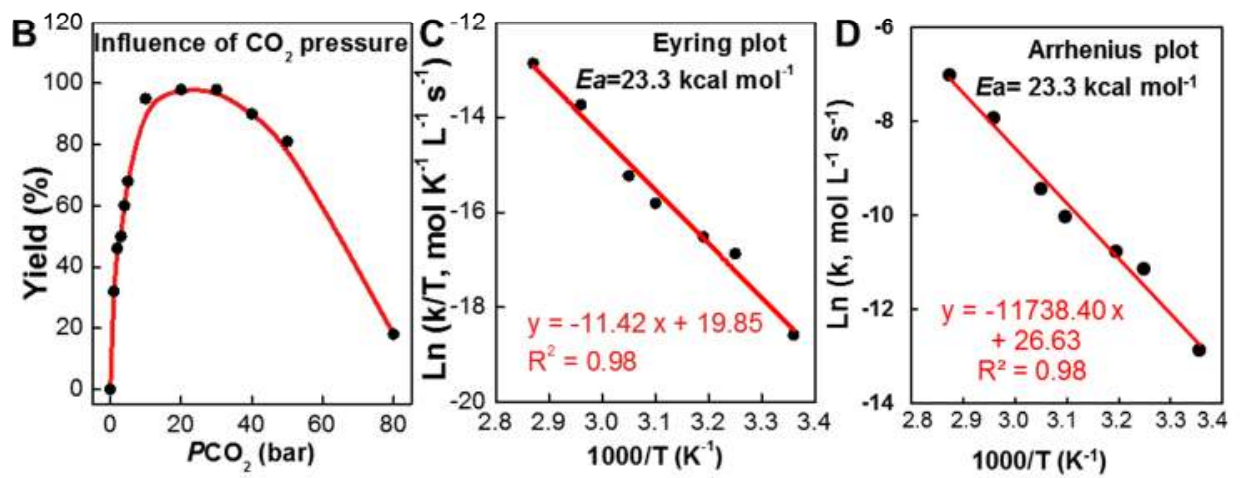

Figure S9: (A) Kinetic data. (B) Influence of $\mathrm{CO}_{2}$ pressure on the yield in the range of 1-80 bar shows an optimum between 10-30 bar. (C, D) The activation energy was determined experimentally by using an Eyring and an Arrhenius plot. 


\section{Role of water}
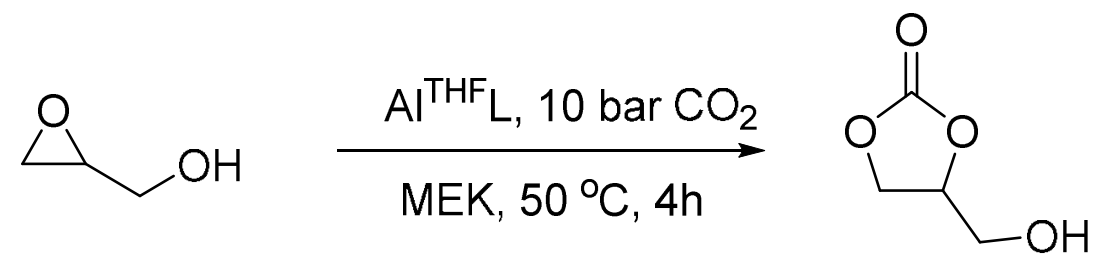

Normal conditions: $62 \%$ yield

Anhydrous conditions $32 \%$ yield

Figure S10: Comparison glycidol carbonate synthesis under normal conditions (i.e., with untreated MEK) and under anhydrous conditions (MEK carefully dried prior to use). 


\section{In situ ATR-IR spectroscopy in solution}

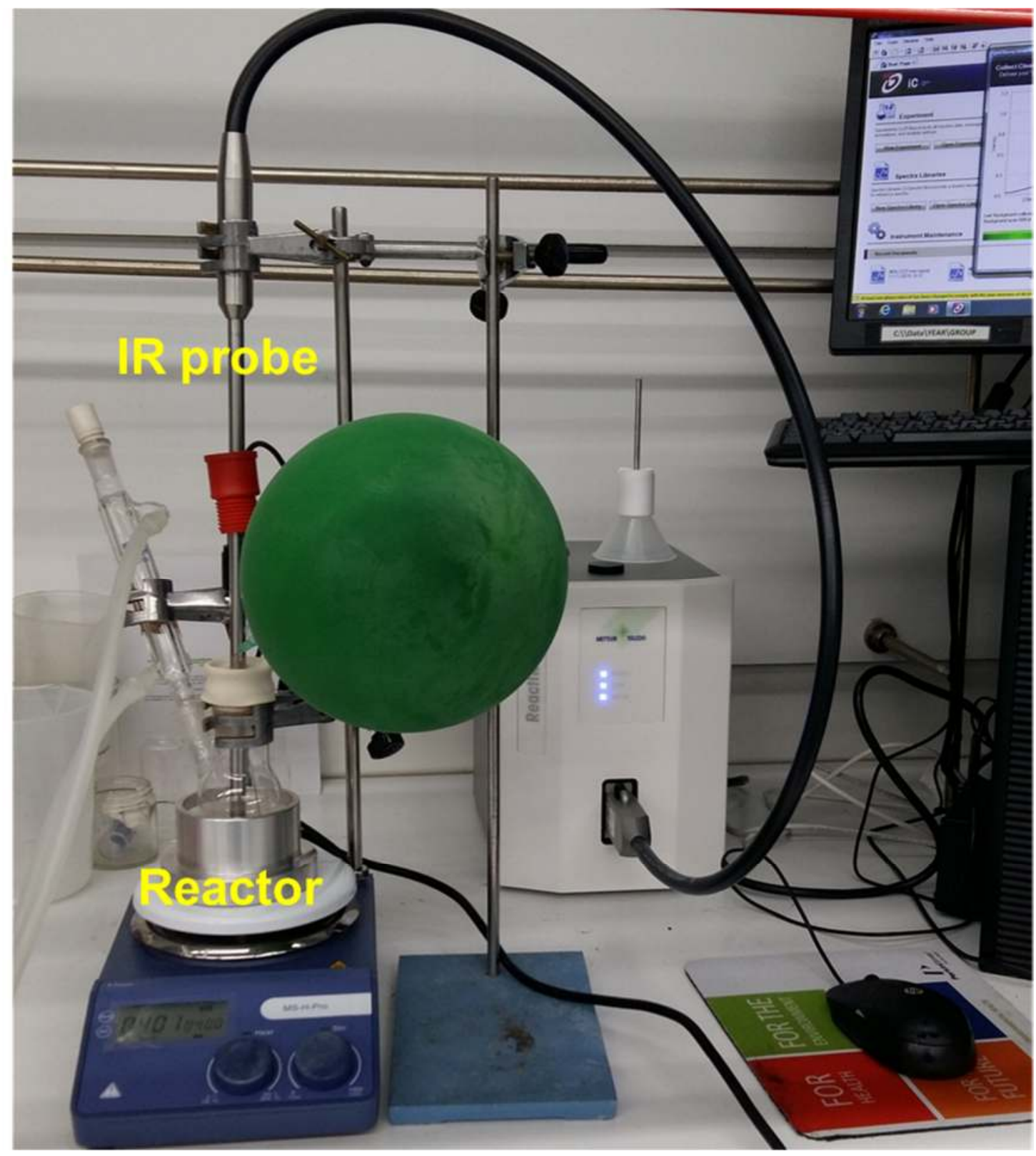


Operando HPIR measurements

High pressure reactor

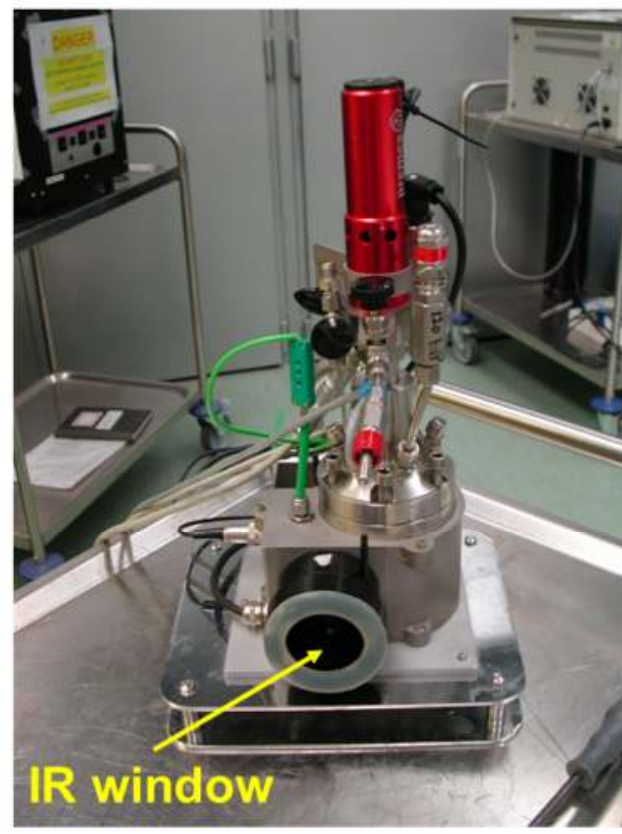

FTIR spectroscopy

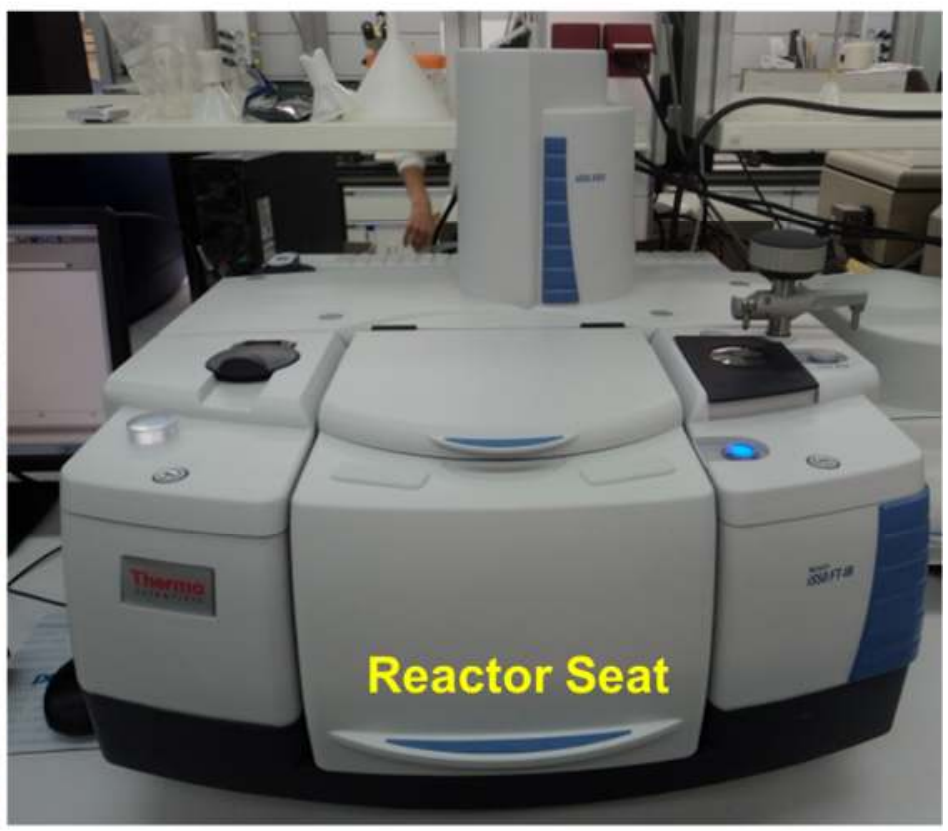


Interactions of $\mathrm{Al}(\mathrm{L})$ with O-containing reagents identified by ATR FTIR spectra
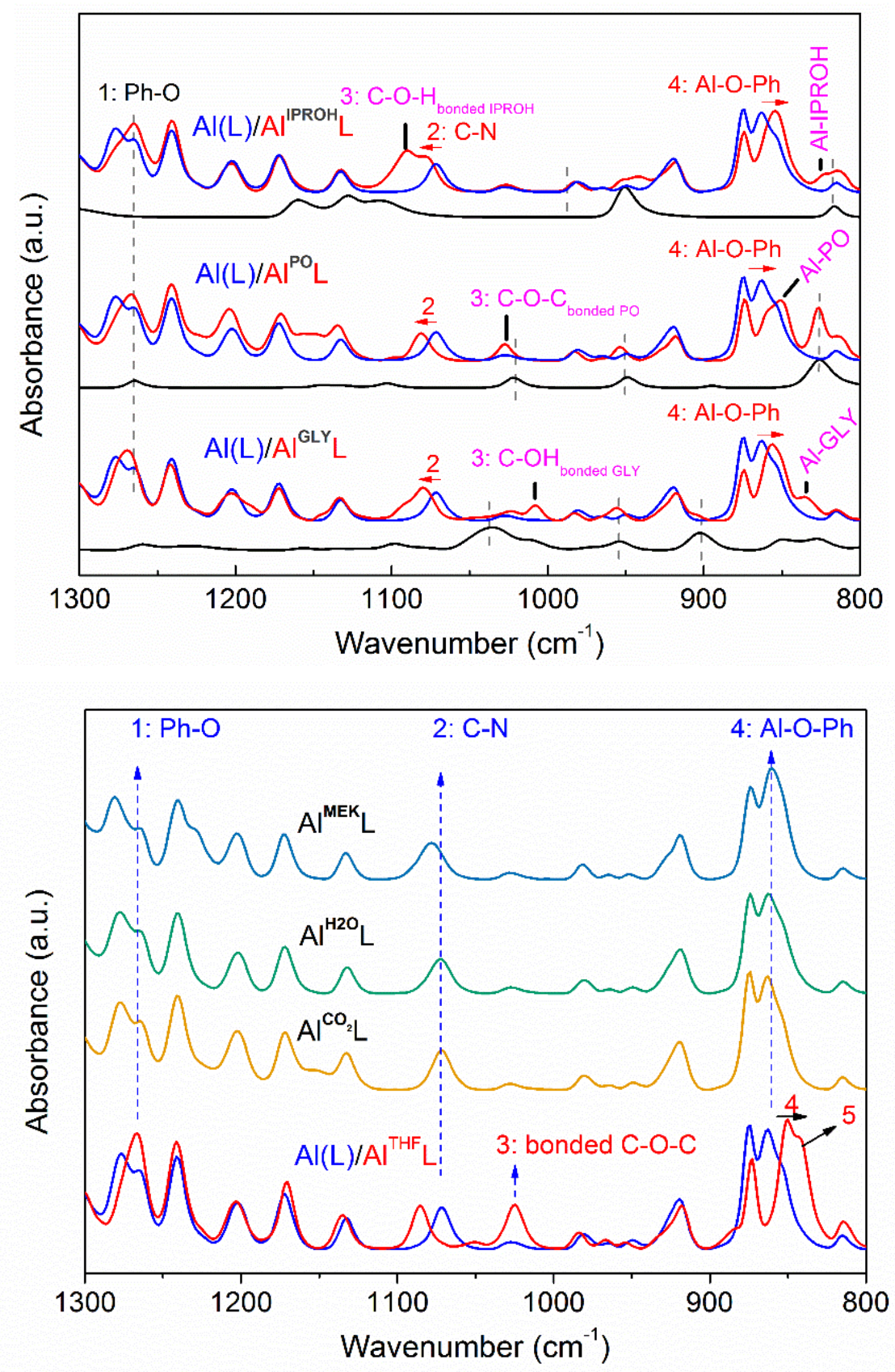

Figure S11. ATR-IR spectroscopic analyses of interactions of $\mathrm{Al}(\mathrm{L})$ catalyst with other reagents. The spectra of $\mathrm{Al}(\mathrm{L})$ and $\mathrm{Al}^{\mathrm{THF}} \mathrm{L}$ were used as references. IPROH: isopropanol; PO: propylene oxide; GLY: glycidol; MEK: 2-butone; THF: tetrahydrofuran. 


\section{X-ray structure for AIGLYL}
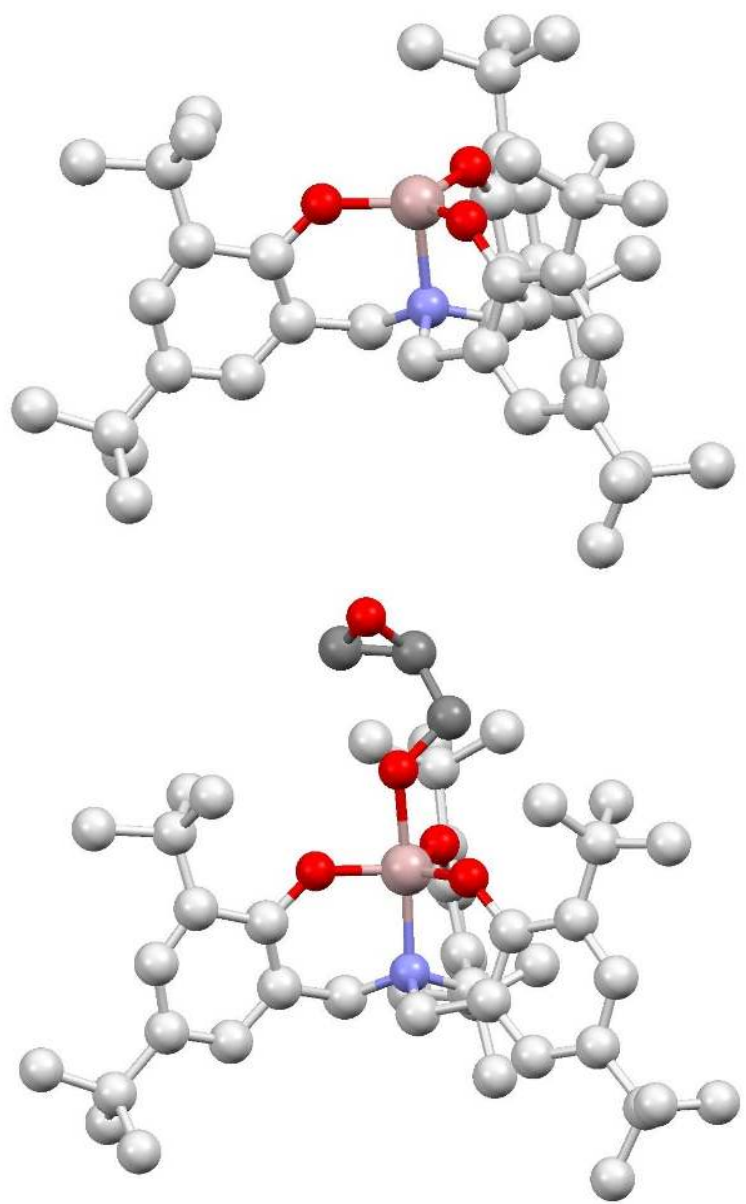

Figure S12. X-ray structure AIGLYL (bottom); for clarity the structure for the Alcomplex without the GLY coordinating is presented at the top. 


\section{Hydrogen bonds between glycidol and water}

The GLY spectrum is featured with several extra bands (highlighted in Figure S13) compared to the simulated spectrum. These features are speculated to originate from the $\mathrm{HB}$ interactions between $\mathrm{GLY}$ and $\mathrm{H}_{2} \mathrm{O}$ present in air and trapped by GLY due to the high hydrophilicity. Upon addition of deuterated water to the GLY sample, the band intensity at the blue-highlighted regions $\left(1650 \mathrm{~cm}^{-1}\right.$ and $953 \mathrm{~cm}^{-}$ $\left.{ }^{1}\right)$ decreases but that of red-colored regions $\left(1098 \mathrm{~cm}^{-1}\right.$ and $\left.850 \mathrm{~cm}^{-1}\right)$ remains unchanged. The reduced intensities at $1650 \mathrm{~cm}^{-1}$ and $953 \mathrm{~cm}^{-1}$ are immediately recovered after 5 min upon exposure to air. These observations support the view that the changes in intensity of the peaks in the blue-highlighted regions influenced by $\mathrm{D}_{2} \mathrm{O}$ addition represent $\mathrm{HB}$ interactions between $\mathrm{GLY}$ and $\mathrm{H}_{2} \mathrm{O}$ (structure 1), whereas the peaks in the red-indicated regions are unaffected by $\mathrm{D}_{2} \mathrm{O}$ addition and thus should be ascribed to intra- and inter-molecular hydrogen bond GLY structures (cf., structures 2 and 3 ).

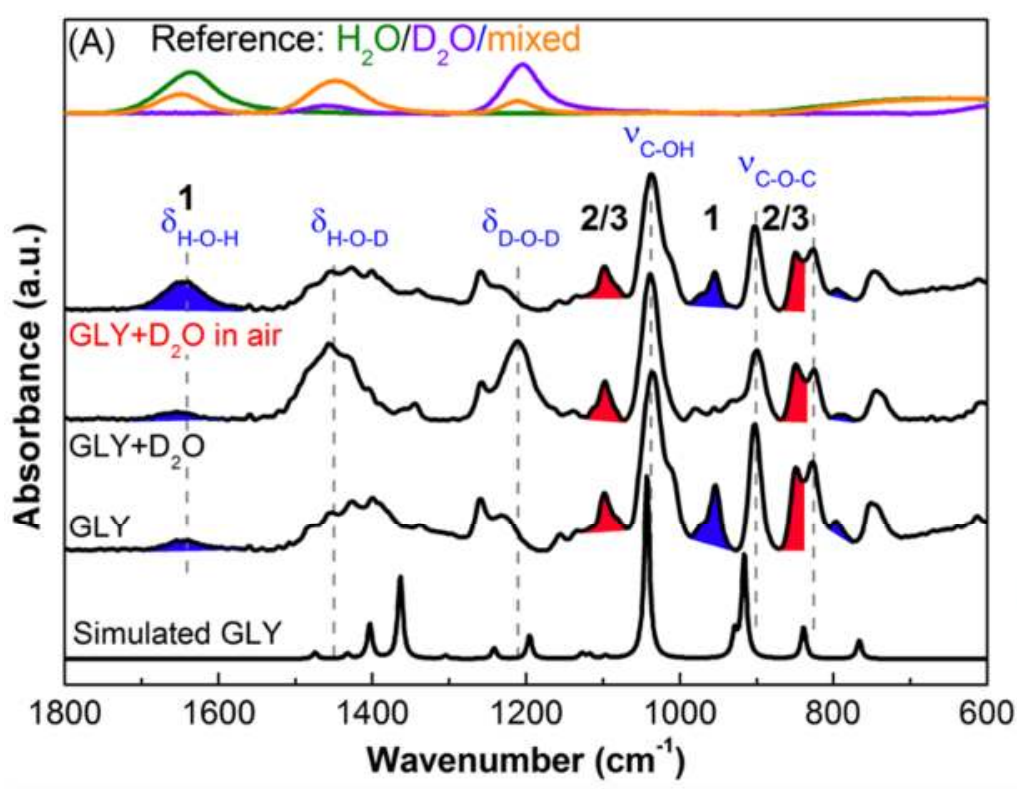<smiles>[CH]1COC2CC2CO1</smiles>

H 1<smiles>C1OC2CC1O2</smiles>

2
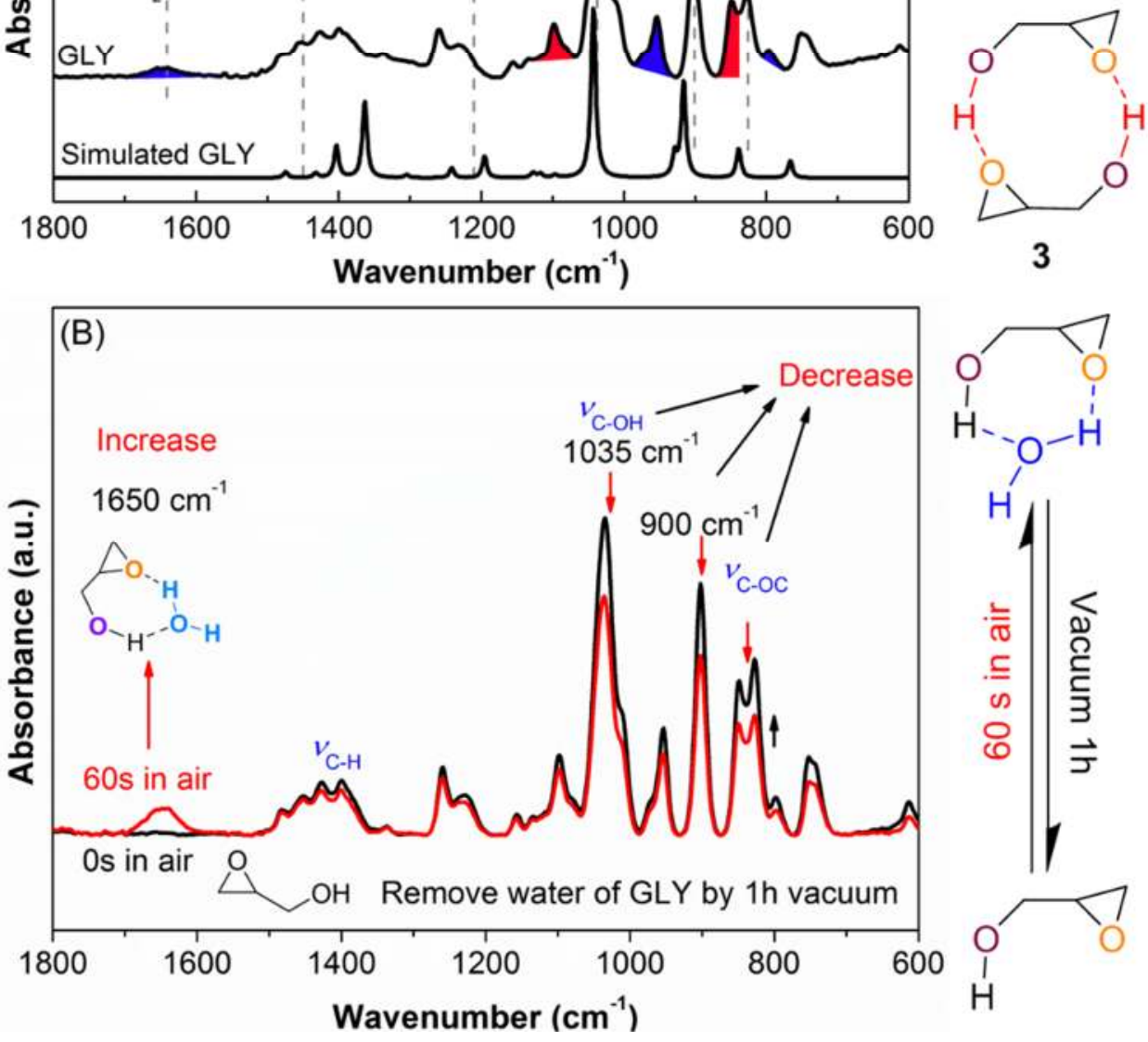
Figure S13. ATR-IR spectroscopic analysis of glycidol-water interactions. (A) Glycidol (GLY) was mixed with 1 equivalent of deuterated water $\left(\mathbf{G L Y}+\mathbf{D}_{\mathbf{2}} \mathbf{O}\right)$ and followed by an exposure to air for 5 min (GLY+D $\mathbf{D}_{2} \mathrm{O}$ in air). $\mathrm{H}_{2} \mathrm{O}, \mathrm{D}_{2} \mathrm{O}$ and their mixtures were used as references. The simulated spectrum was calculated with B3PW91/6-311G $(2 d, 2 p)$, and the calculated vibrational frequency is scaled by 0.965 . (B) Anhydrous GLY was obtained by the treatment of vacuum for $1 \mathrm{~h}$ after which it was exposed to air for $60 \mathrm{~s}$. All samples were analyzed by ATR-IR measurements. 


\section{$\mathrm{CO}_{2}$ trapping measurements from other substrates}

$$
\text { ( }
$$

Figure S14. Trapping of $\mathrm{CO}_{2}$ by GLY (top) leads to a relatively stable species that slowly loses $\mathrm{CO}_{2}$ over time upon flushing the system with argon. Subsequent addition of TBAI initiates product formation which can be accelerated by introducing Al-catalyst to the reaction mixture. The same phenomenon could not be observed when the same procedure was used with other epoxides and the Alcomplex. 
Fingerprint regions of the in-situ ATR-IR spectra
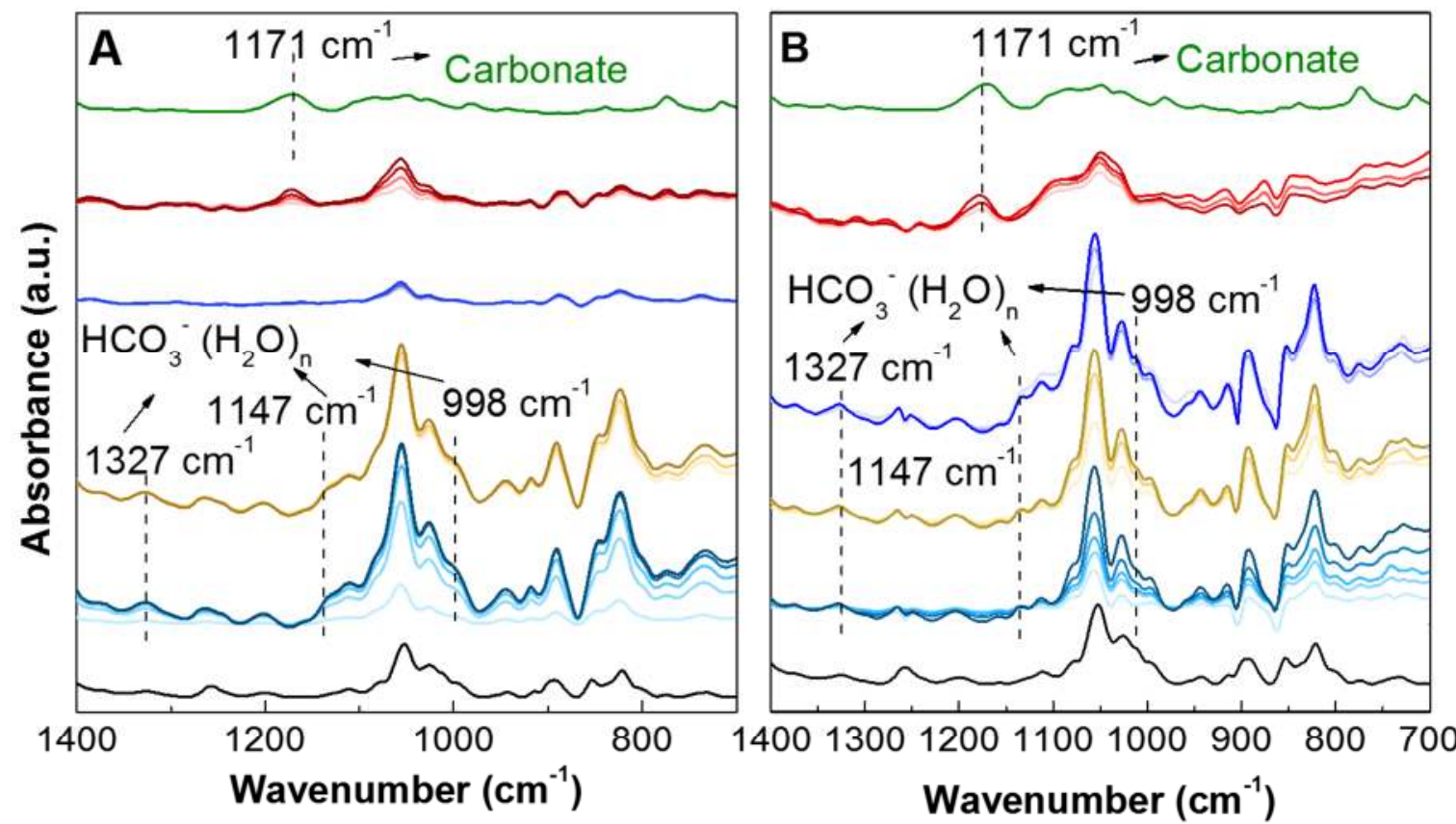

\section{Sequences}
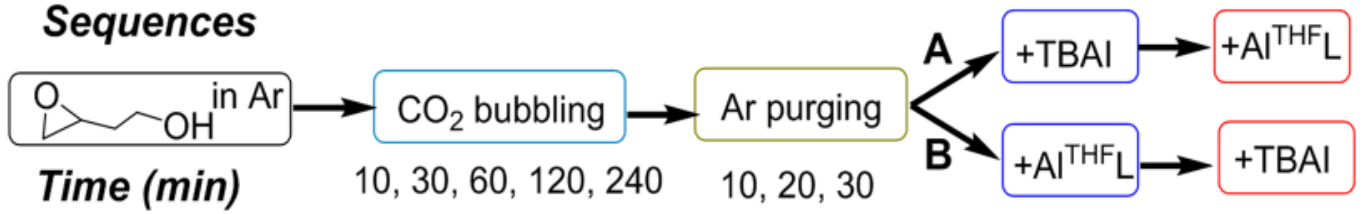

$10,30,5010,60,120,240$

Figure S15. Fingerprint regions of the in-situ ATR-IR spectra related to Figure $6 \mathrm{~A}$ and 6B. Characteristic band assignments of the structure of $\mathrm{HCO}-(\mathrm{H} 2 \mathrm{O}) n$ are based on those provided in reference 14 . 


\section{Ligand assisted substrate activation}

One of the phenolate groups from the ligand aids in the activation of the substrate by deprotonating the alcohol from glycidol. This causes a decoordination of the phenolate from the metal and results in the formation of an aluminum bound alkoxide species (see Figure S16). Similar type of non-innocent ligand behavior where the ligand can aid in proton shuttling has been previously reported for aluminum (III) complexes bearing tridentate bis(amino)pyridine ligands. ${ }^{[15]}$ In addition, we recently reported on the isolation of a vanadium-PO complex where one of the phenolate ligands was able to act as an internal nucleophile thereby ring-opening a coordinating epoxide..$^{[16]}$

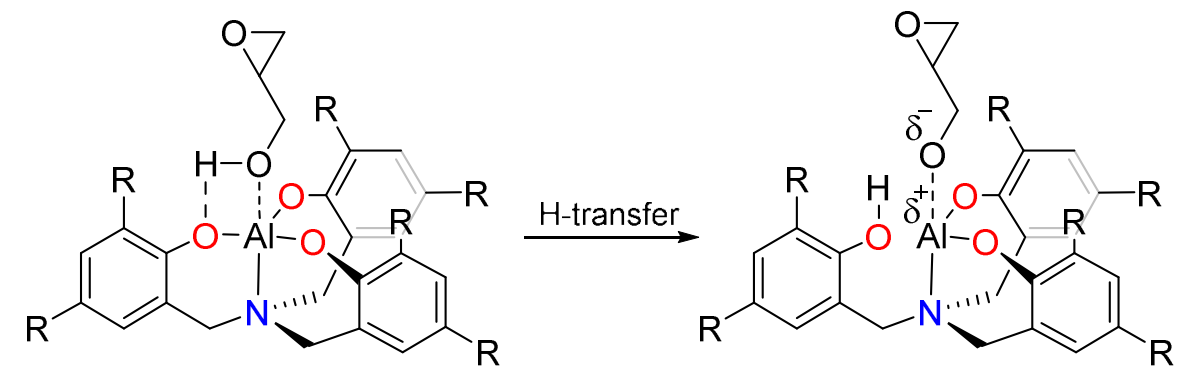

Figure S16. Formation of a metal-bound alkoxide via deprotonation of the substrate by the ligand. 


\section{Peak-fitting of operando HP-IR spectra}
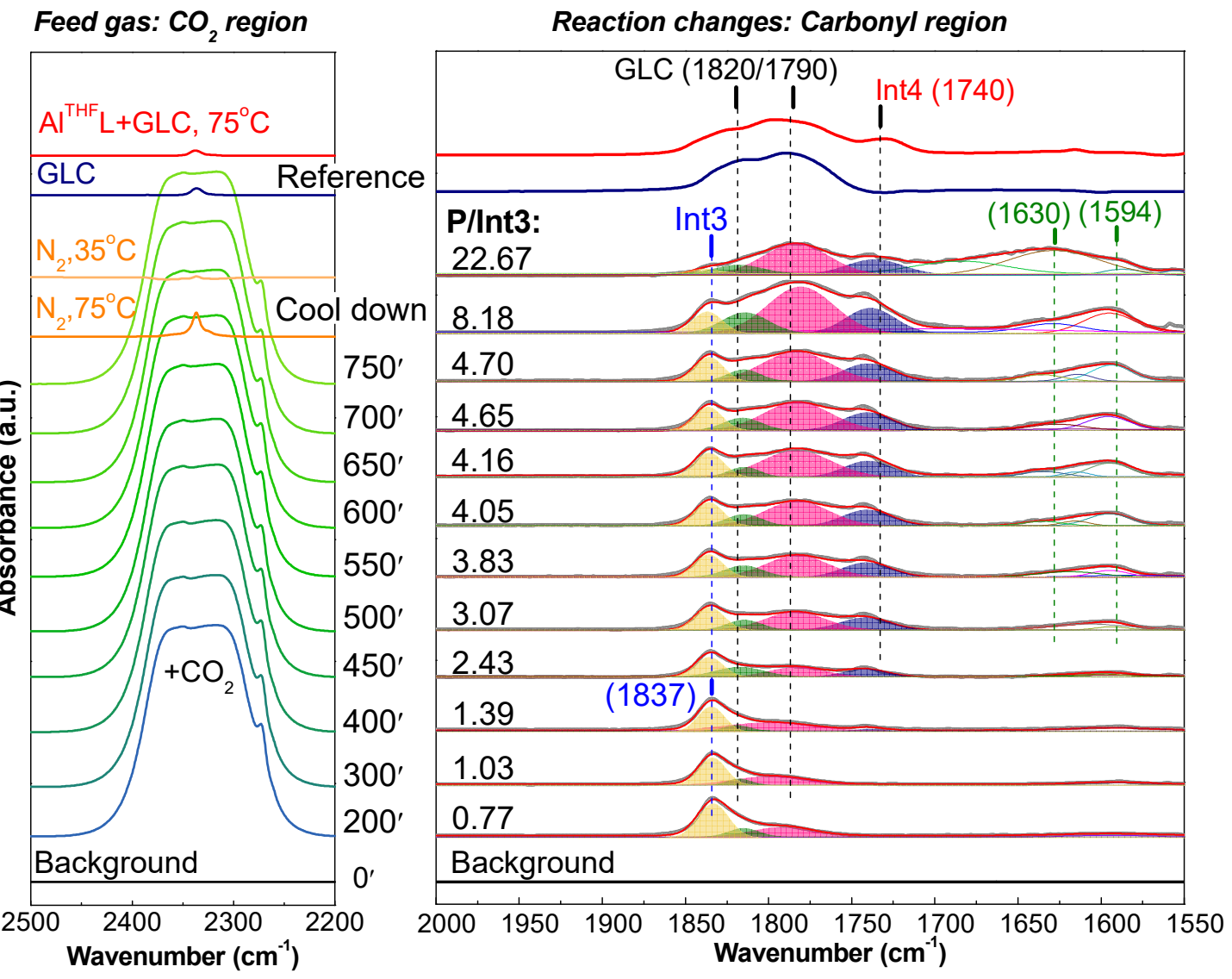

Figure S17. Deconvolution for the spectra presented in Figure 6. Each spectrum at the carbonyl region is fitted into several components (shadow areas) and the observed survey spectrum is shown in gray, whereas the fitted survey spectrum is shown in red. The results of the peak-fitting analysis are given in Table S5. 'P/Int3' represents 'the ratio of the peak area of products (GLC + Int4) to Int3'. As reported earlier, the peaks at 1820 and $1790 \mathrm{~cm}-1$ could be regarded as two cis-trans GLC isomers.[17] Further to this, a control experiment combining AITHFL with GLC (top of Figure S17) where the aluminum complex and product were mixed in a 1:4 ratio under the same reaction conditions (75 oC, 10 bar N2) shows three peaks at 1740,1790 and $1820 \mathrm{~cm}-1$, confirming that they indeed arise from GLC. 
Table S5 Peak-fitting parameters of operando HP-IR spectra in Figure S16

\begin{tabular}{|c|c|c|c|c|c|}
\hline Sample & Peak name & Position (cm-1) & FWHM (cm-1) & Peak Area & $\mathrm{P} / \mathrm{Int3}$ \\
\hline \multirow{4}{*}{ CO2-200' } & Int4 & - & - & 0.00 & \multirow{4}{*}{0.77} \\
\hline & GLC & 1792.42 & 41.67 & 1.75 & \\
\hline & GLC & 1815.04 & 20.82 & 0.77 & \\
\hline & Int3 & 1833.34 & 21.82 & 3.26 & \\
\hline \multirow{4}{*}{ CO2-300' } & Int4 & - & - & 0.00 & \multirow{4}{*}{1.03} \\
\hline & GLC & 1799.19 & 51.58 & 1.94 & \\
\hline & GLC & 1817.76 & 16.51 & 0.45 & \\
\hline & Int3 & 1833.91 & 19.42 & 2.32 & \\
\hline \multirow{4}{*}{ CO2-400' } & Int4 & 1739.36 & 20.76 & 0.24 & \multirow{4}{*}{1.39} \\
\hline & GLC & 1800.76 & 62.23 & 2.23 & \\
\hline & GLC & 1819.07 & 18.20 & 0.44 & \\
\hline & Int3 & 1834.63 & 18.89 & 2.09 & \\
\hline \multirow{4}{*}{ CO2-450' } & Int4 & 1742.12 & 30.34 & 1.04 & \multirow{4}{*}{2.43} \\
\hline & GLC & 1782.75 & 38.21 & 1.66 & \\
\hline & GLC & 1817.38 & 34.49 & 1.48 & \\
\hline & Int3 & 1835.83 & 19.76 & 1.72 & \\
\hline \multirow{4}{*}{ CO2-500' } & Int4 & 1742.14 & 34.73 & 1.89 & \multirow{4}{*}{3.07} \\
\hline & GLC & 1784.11 & 42.53 & 3.35 & \\
\hline & GLC & 1814.66 & 24.19 & 1.06 & \\
\hline & Int3 & 1835.66 & 20.54 & 2.05 & \\
\hline \multirow{4}{*}{ CO2-550' } & Int4 & 1741.32 & 33.89 & 2.18 & \multirow{4}{*}{3.83} \\
\hline & GLC & 1782.92 & 44.06 & 4.19 & \\
\hline & GLC & 1815.19 & 26.47 & 1.31 & \\
\hline & Int3 & 1836.15 & 20.19 & 2.01 & \\
\hline \multirow{4}{*}{ CO2-600' } & Int4 & 1741.10 & 33.74 & 2.36 & \multirow{4}{*}{4.05} \\
\hline & GLC & 1783.01 & 45.38 & 4.86 & \\
\hline & GLC & 1814.90 & 24.89 & 1.25 & \\
\hline & Int3 & 1836.08 & 20.28 & 2.09 & \\
\hline
\end{tabular}

Table S5 continued

\begin{tabular}{|l|l|l|l|l|l|}
\hline Sample & Peak name & Position (cm-1) & FWHM (cm-1) & Peak Area & P/Int3 \\
\hline
\end{tabular}




\begin{tabular}{|c|c|c|c|c|c|}
\hline \multirow{4}{*}{ CO2-650' } & \begin{tabular}{|l|}
$\ln 4$ \\
\end{tabular} & 1740.37 & 33.42 & 2.37 & \multirow{4}{*}{4.16} \\
\hline & GLC & 1783.48 & 49.31 & 5.73 & \\
\hline & GLC & 1814.79 & 21.77 & 0.96 & \\
\hline & Int3 & 1835.88 & 20.45 & 2.18 & \\
\hline \multirow{4}{*}{ CO2-700' } & \begin{tabular}{|l|} 
Int4 \\
\end{tabular} & 1739.08 & 31.87 & 2.05 & \multirow{4}{*}{4.65} \\
\hline & GLC & 1784.03 & 57.83 & 7.12 & \\
\hline & GLC & 1815.04 & 17.02 & 0.53 & \\
\hline & \begin{tabular}{|l|} 
Int3 \\
\end{tabular} & 1835.72 & 20.27 & 2.09 & \\
\hline \multirow{4}{*}{ CO2-750' } & Int4 & 1741.00 & 35.12 & 2.84 & \multirow{4}{*}{4.70} \\
\hline & GLC & 1783.18 & 46.18 & 5.96 & \\
\hline & GLC & 1814.90 & 24.25 & 1.33 & \\
\hline & Int3 & 1836.11 & 20.24 & 2.16 & \\
\hline \multirow{4}{*}{ N2-75oC } & Int4 & 1738.88 & 34.85 & 3.85 & \multirow{4}{*}{8.18} \\
\hline & GLC & 1780.86 & 46.89 & $\overline{9.54}$ & \\
\hline & GLC & 1814.28 & 33.03 & 3.00 & \\
\hline & Int3 & 1836.93 & 21.07 & 2.00 & \\
\hline \multirow{4}{*}{$\mathrm{N} 2-35 \mathrm{oC}$} & Int4 & 1736.44 & 39.75 & 2.63 & \multirow{4}{*}{22.67} \\
\hline & GLC & 1783.61 & 45.98 & 6.24 & \\
\hline & GLC & 1818.00 & 32.59 & 1.22 & \\
\hline & \begin{tabular}{|l|}
$\ln 3$ \\
\end{tabular} & 1838.53 & 21.99 & 0.45 & \\
\hline
\end{tabular}

'P/Int3' = [Peak area (GLC) + Peak area (Int4)]/Peak area (Int3) 


\section{NMR Spectral Data for Compounds B-F Compound $\mathrm{B}^{[18]}$}

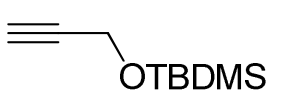

To a solution of propargylic alcohol $(1.12 \mathrm{~g}, 20.0 \mathrm{mmol})$ in DCM $(40 \mathrm{~mL})$ was added TBDMS-Cl $(3.16 \mathrm{~g}, 21.0 \mathrm{mmol})$ and imidazole $(2.72 \mathrm{~g}, 40.0 \mathrm{mmol})$. After stirring for 16 hours the washed with water, dried over $\mathrm{MgSO}_{4}$ and concentrated under vacuum to yield the product as a slighly yellow liquid in $99 \%$ yield $(3.37 \mathrm{~g}, 19.8 \mathrm{mmol}) .{ }^{1} \mathrm{H} \mathrm{NMR}\left(400 \mathrm{MHz}, \mathrm{CDCl}_{3}\right)$ $\delta 4.33(\mathrm{~d}, \mathrm{~J}=2.4 \mathrm{~Hz}, 2 \mathrm{H}), 2.40(\mathrm{t}, \mathrm{J}=2.4 \mathrm{~Hz}, 1 \mathrm{H}), 0.93(\mathrm{~s}, 9 \mathrm{H}), 0.14(\mathrm{~s}, 6 \mathrm{H}) .{ }^{13} \mathrm{C}$ NMR $\left(101 \mathrm{MHz}, \mathrm{CDCl}_{3}\right) \delta 82.42,72.81,51.50,25.78,18.27,-5.21$.

\section{Compound $\mathrm{C}^{[3]}$}

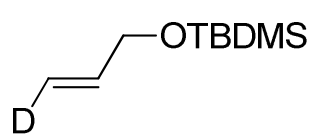

In a dried schlenk-flask protected from light by aluminum foil, were added $\mathrm{Cp}_{2} \mathrm{ZrCl}_{2}(2.92 \mathrm{~g}, 10.0 \mathrm{mmol})$ and THF $(60 \mathrm{~mL})$ under nitrogen atmosphere. $\mathrm{LiEt}_{3} \mathrm{BH}(10 \mathrm{~mL}, 10.0 \mathrm{mmol})$ was slowly added and stirred for 45 mins at room temperature before adding the alkyne $\mathbf{B}\left(1.70 \mathrm{~g}, 2.10 \mathrm{~mL}, 10.0 \mathrm{mmol}\right.$, previously distilled over $\left.\mathrm{CaH}_{2}\right)$ and stirring for an additional 1 hour. Afterwards $\mathrm{D}_{2} \mathrm{O}(2.0 \mathrm{~mL})$ was added and stirred for 30 mins. Ether was added after the reaction and the mixture was dried over $\mathrm{MgSO}_{4}$ and the solvent evaporated. The crude product was purified by neutral alumina column chromatography (hexane) to obtain the product as a colorless liquid in $65 \%$ yield $(1.13 \mathrm{~g}, 6.5 \mathrm{mmol}) .{ }^{1} \mathrm{H}$ NMR $\left(500 \mathrm{MHz}, \mathrm{CDCl}_{3}\right) \delta 5.99-5.89(\mathrm{~m}, 1 \mathrm{H})$, $5.28(\mathrm{~m}, 1 \mathrm{H}), 4.20(\mathrm{~m}, 2 \mathrm{H}), 0.94(\mathrm{~s}, 9 \mathrm{H}), 0.10(\mathrm{~s}, 6 \mathrm{H}) \cdot{ }^{13} \mathrm{C} \mathrm{NMR}\left(126 \mathrm{MHz}, \mathrm{CDCl}_{3}\right)$ $\delta 137.40,113.65,64.07,25.93,18.41,-5.26$.

\section{Compound D}

To a solution of C $(0.80 \mathrm{~g}, 4.6 \mathrm{mmol})$ in $\mathrm{DCM}(10 \mathrm{~mL})$ was
added m-CPBA $(0.95 \mathrm{~g}, 5.5 \mathrm{mmol})$ and the mixture was than stirred for 24 hours at $45^{\circ} \mathrm{C}$. After the reaction the mixture was isolated by basic alumina column chromatography (Hexane: $\mathrm{Et}_{2} \mathrm{O}, 10: 1$ ) to yield the product as a colorless liquid in $83 \%$ yield $(0.73 \mathrm{~g}, 3.8 \mathrm{mmol}) .{ }^{1} \mathrm{H}$ NMR $\left(500 \mathrm{MHz}, \mathrm{CDCl}_{3}\right) \delta 3.87$ $(\mathrm{m}, 1 \mathrm{H}), 3.68(\mathrm{~m}, 1 \mathrm{H}), 3.12-3.08(\mathrm{~m}, 1 \mathrm{H}), 2.64(\mathrm{~d}, \mathrm{~J}=2.7 \mathrm{~Hz}, 1 \mathrm{H}), 0.92(\mathrm{~s}, 9 \mathrm{H})$, $0.10(\mathrm{~d}, \mathrm{~J}=4.5 \mathrm{~Hz}, 6 \mathrm{H}) .{ }^{13} \mathrm{C} \mathrm{NMR}\left(126 \mathrm{MHz}, \mathrm{CDCl}_{3}\right) \delta 63.73,52.33,44.36$, $25.86,18.36,-5.32$.

\section{Compound $E^{[19]}$}

To a solution of $\mathbf{D}(0.36 \mathrm{~g}, 1.9 \mathrm{mmol})$ in THF $(1 \mathrm{~mL})$ was added a DOH $1 \mathrm{M}$ solution of TBAF in THF $(2.0 \mathrm{~mL}, 2.0 \mathrm{mmol})$ and stirred for 18 hours at room temperature. After the reaction the mixture was purified by neutral alumina column chromatography (Pentane: $\mathrm{Et}_{2} \mathrm{O}, 1: 1$ ) to yield the final product as colorless liquid in $99 \%$ yield $(0.14 \mathrm{~g}, 1.9 \mathrm{mmol}) .{ }^{1} \mathrm{H} \mathrm{NMR}\left(500 \mathrm{MHz}, \mathrm{CDCl}_{3}\right) \delta$ $3.89(\mathrm{dd}, \mathrm{J}=12.7,2.5 \mathrm{~Hz}, 1 \mathrm{H}), 3.52(\mathrm{dd}, \mathrm{J}=12.7,4.9 \mathrm{~Hz}, 1 \mathrm{H}), 3.23-3.11(\mathrm{~m}$, $1 \mathrm{H}), 3.11(\mathrm{~m}, 1 \mathrm{H}), 2.68(\mathrm{~d}, \mathrm{~J}=2.8 \mathrm{~Hz}, 1 \mathrm{H}) .{ }^{13} \mathrm{C} \mathrm{NMR}\left(126 \mathrm{MHz}, \mathrm{CDCl}_{3}\right) \delta 62.07$, $52.36,44.02,25.56,-3.74$. 


\section{Compound F}

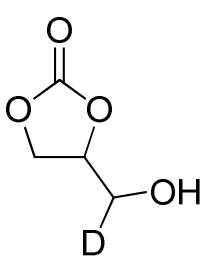

This compound was obtained following the general catalytic procedure described in this paper (page S10). After the reaction the solvent was evaporated and the mixture was purified by silica gel column chromatography to yield the final product as slightly yellow oil in $93 \%$ yield. ${ }^{1} \mathrm{H} \mathrm{NMR}\left(500 \mathrm{MHz}, \mathrm{CDCl}_{3}\right) \delta 4.83(\mathrm{~m}, 1 \mathrm{H}), 4.55(\mathrm{t}$, $\mathrm{J}=8.4 \mathrm{~Hz}, 1 \mathrm{H}), 4.48(\mathrm{~m}, 1 \mathrm{H}), 4.00(\mathrm{~m} \mathrm{1H}), 2.39(\mathrm{~d}, \mathrm{~J}=5.6 \mathrm{~Hz}, 1 \mathrm{H}) .{ }^{13} \mathrm{C} \mathrm{NMR}$ $\left(126 \mathrm{MHz}, \mathrm{CDCl}_{3}\right) \delta 155.12,76.38,65.69,61.39$. 
${ }^{1} \mathrm{H}-\mathrm{NMR}$ and ${ }^{13} \mathrm{C}-\mathrm{NMR}$ spectra of compounds $\mathrm{B}-\mathrm{F}$ Compound B

${ }^{1} \mathrm{H}$ NMR spectrum $\left(\mathrm{CDCl}_{3}\right)$
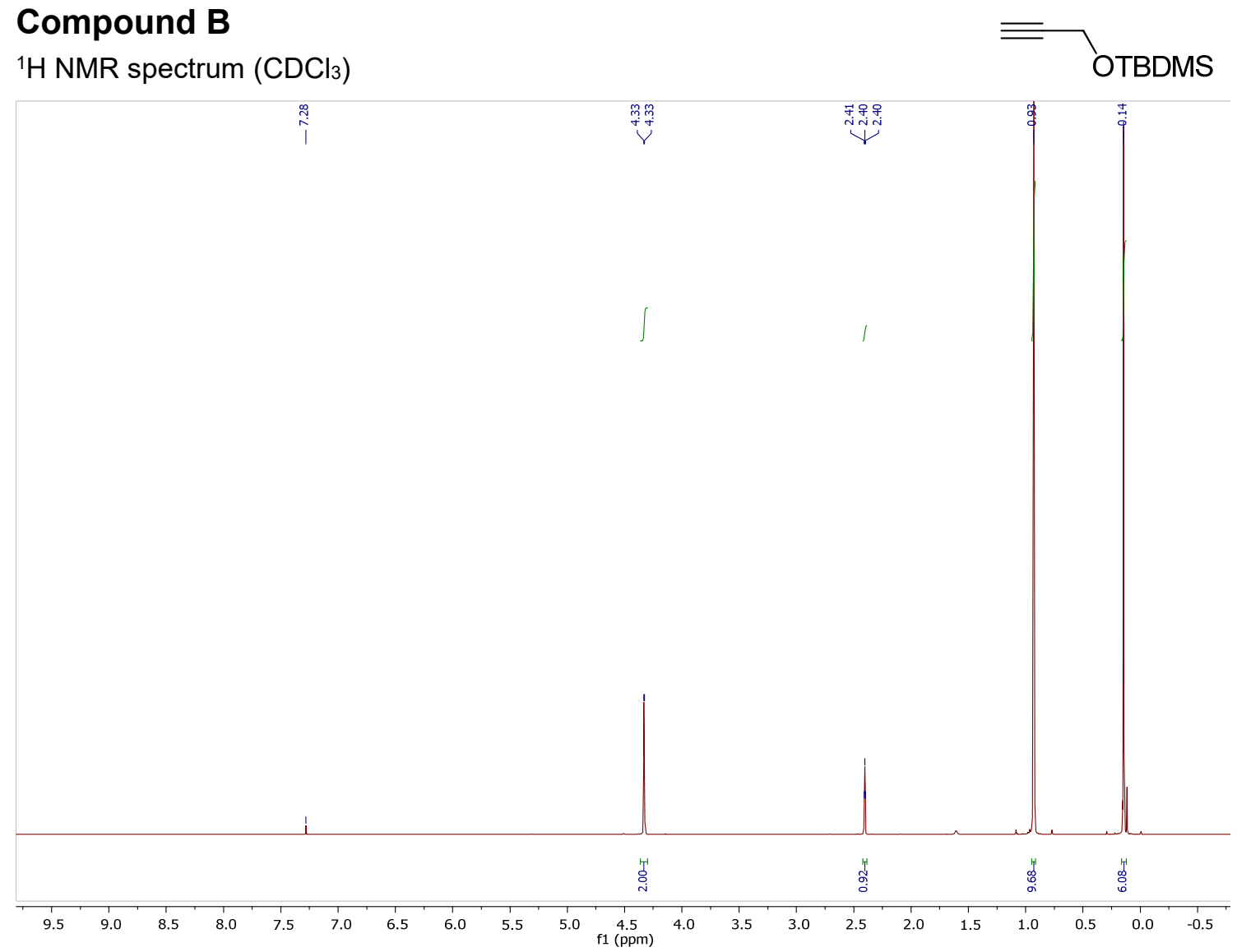

${ }^{13} \mathrm{C}-\mathrm{NMR}$ spectrum $\left(\mathrm{CDCl}_{3}\right)$

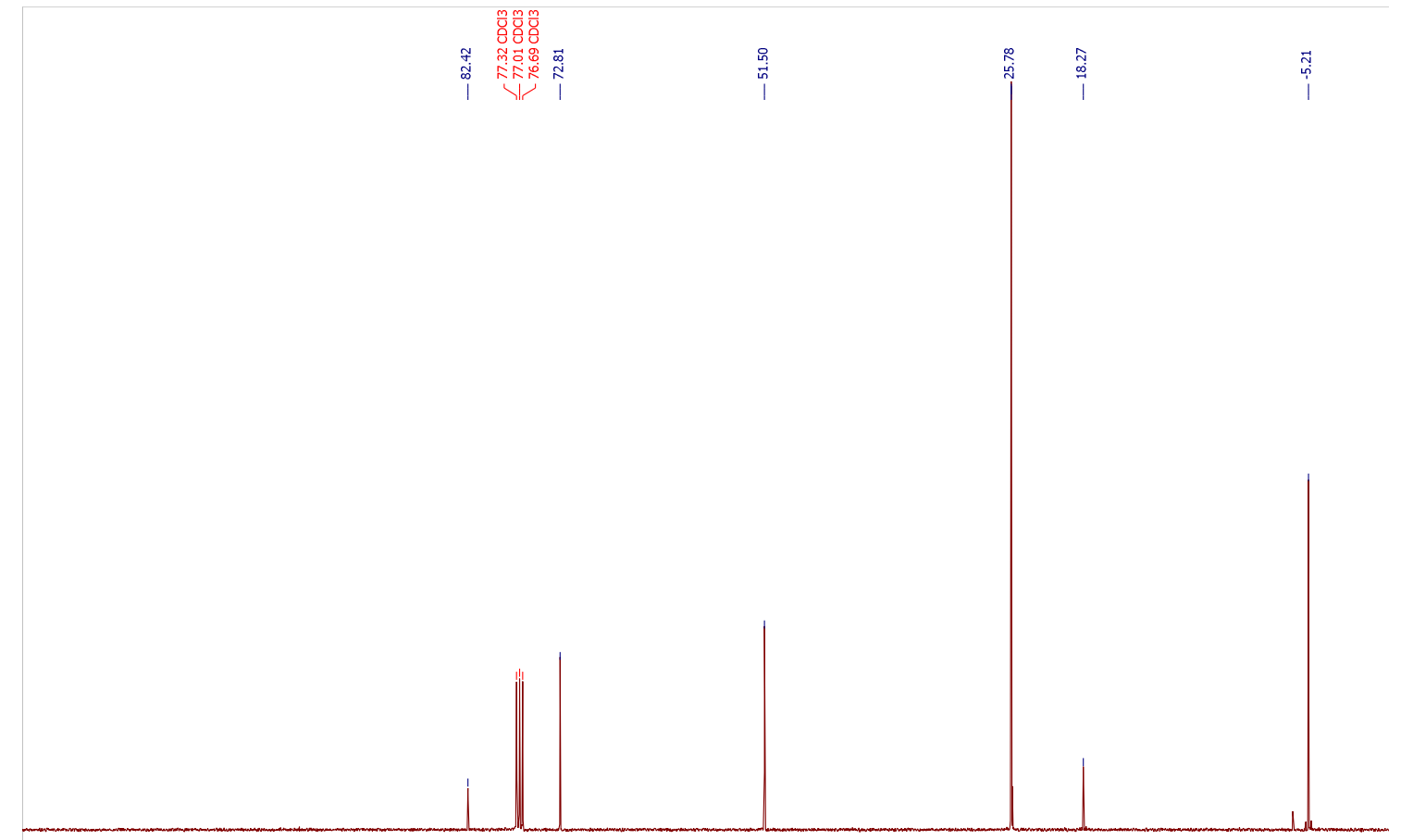

$\begin{array}{llllllllllllllllllllllllllllllllllllllllllll}125 & 120 & 115 & 110 & 105 & 100 & 95 & 90 & 85 & 80 & 75 & 70 & 65 & 60 & 55 & 50 & 45 & 40 & 35 & 30 & 25 & 20 & 15 & 10 & 5 & 0 & -5 & -10\end{array}$ 


\section{Compound C}

${ }^{1} \mathrm{H}$ NMR spectrum $\left(\mathrm{CDCl}_{3}\right)$
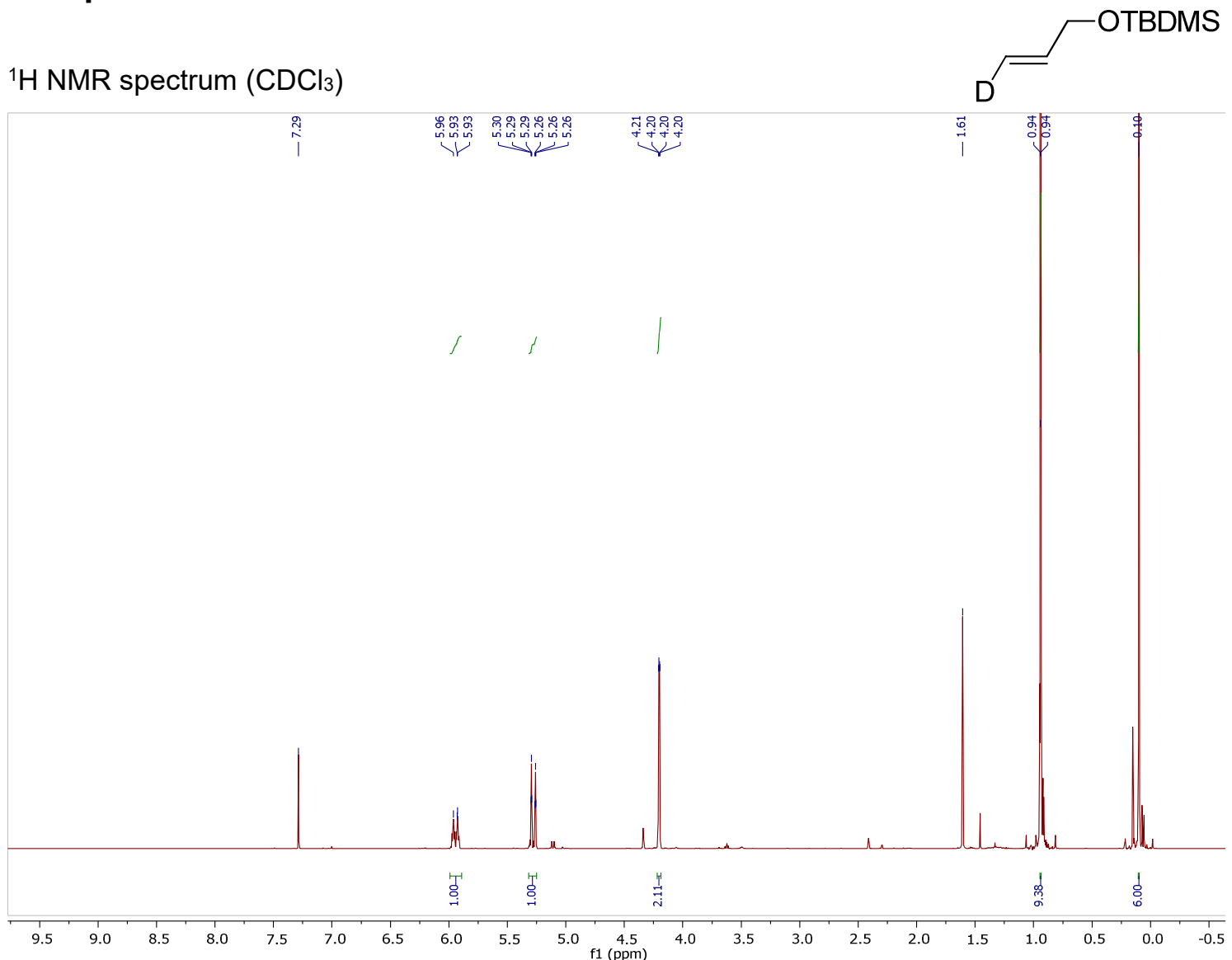

${ }^{13} \mathrm{C}$-NMR spectrum $\left(\mathrm{CDCl}_{3}\right)$

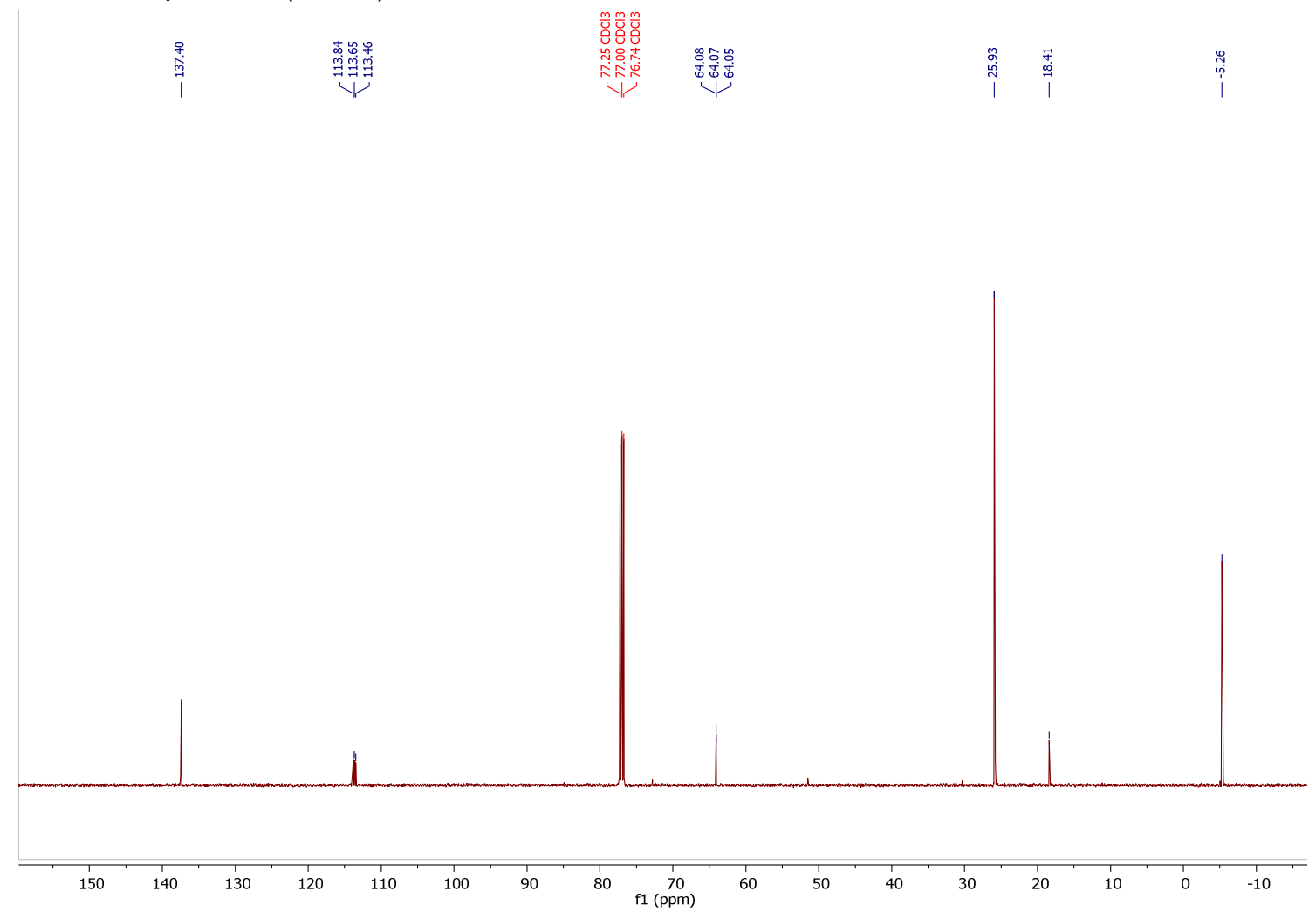


Compound D

${ }^{1} \mathrm{H}$ NMR spectrum $\left(\mathrm{CDCl}_{3}\right)$
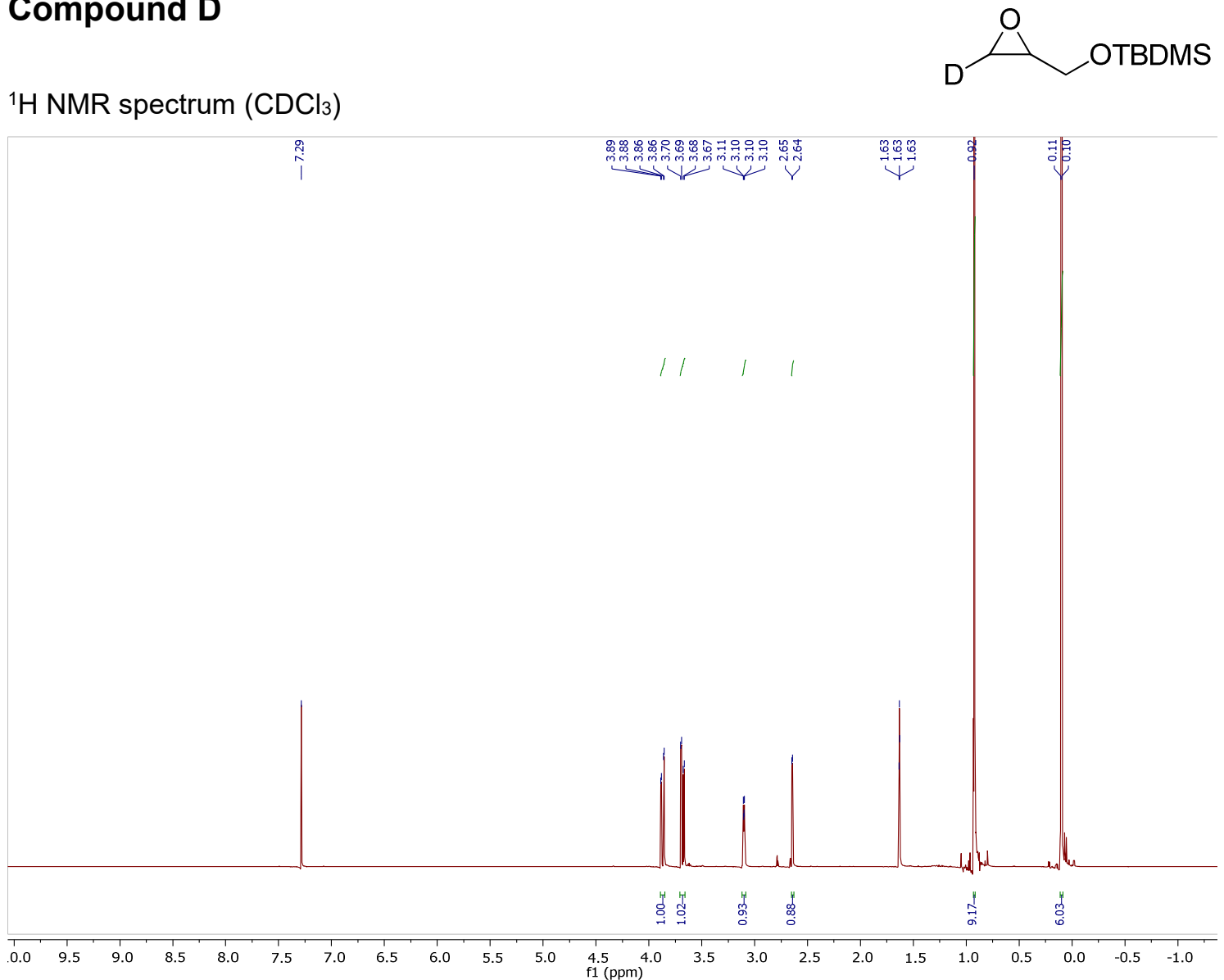

${ }^{13} \mathrm{C}$-NMR spectrum $\left(\mathrm{CDCl}_{3}\right)$

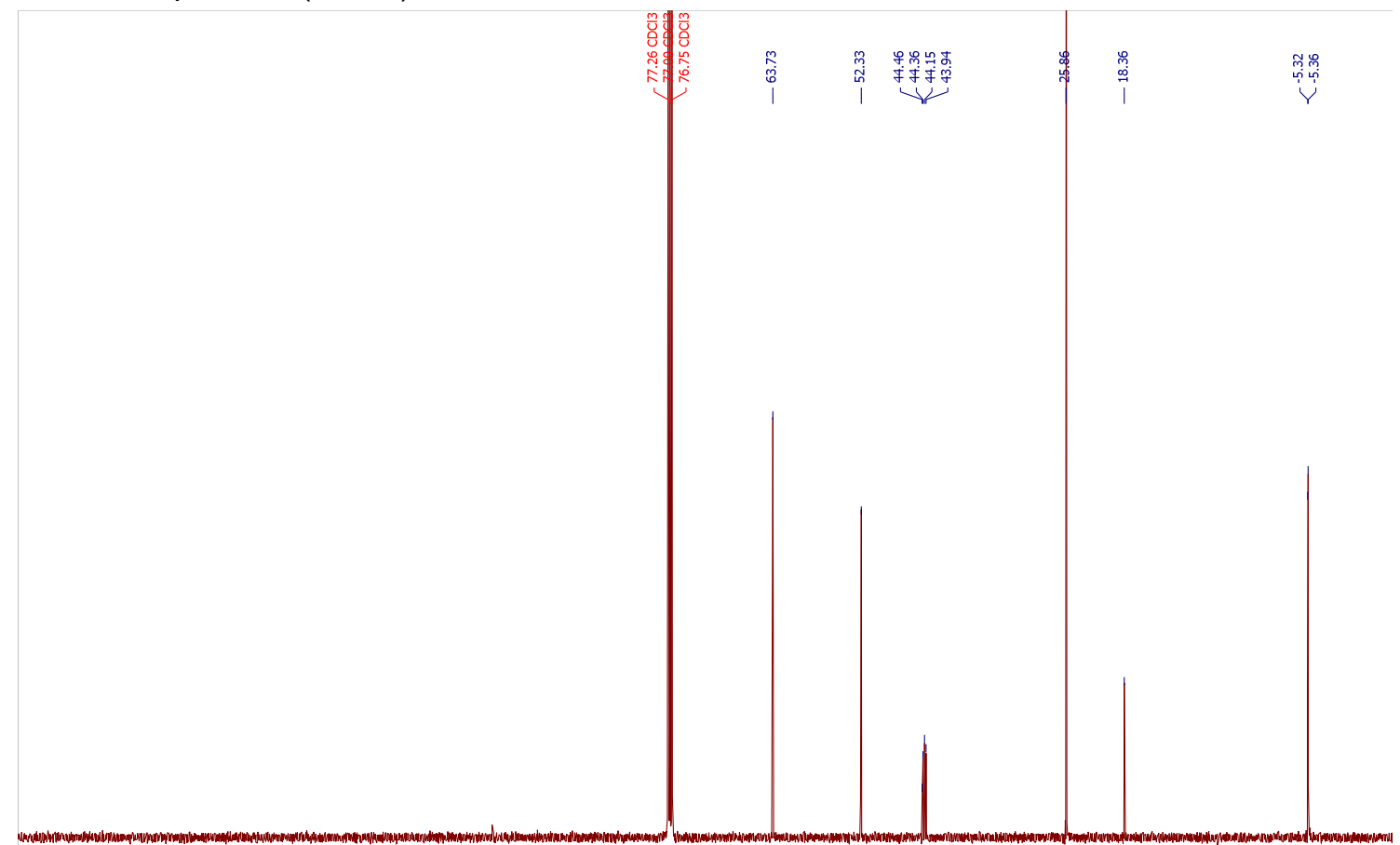

160

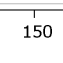

140

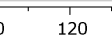

110

$80 \begin{array}{r}70 \\ \mathrm{f} 1(\mathrm{ppm})\end{array}$ 
Compound E

${ }^{1} \mathrm{H}$ NMR spectrum $\left(\mathrm{CDCl}_{3}\right)$

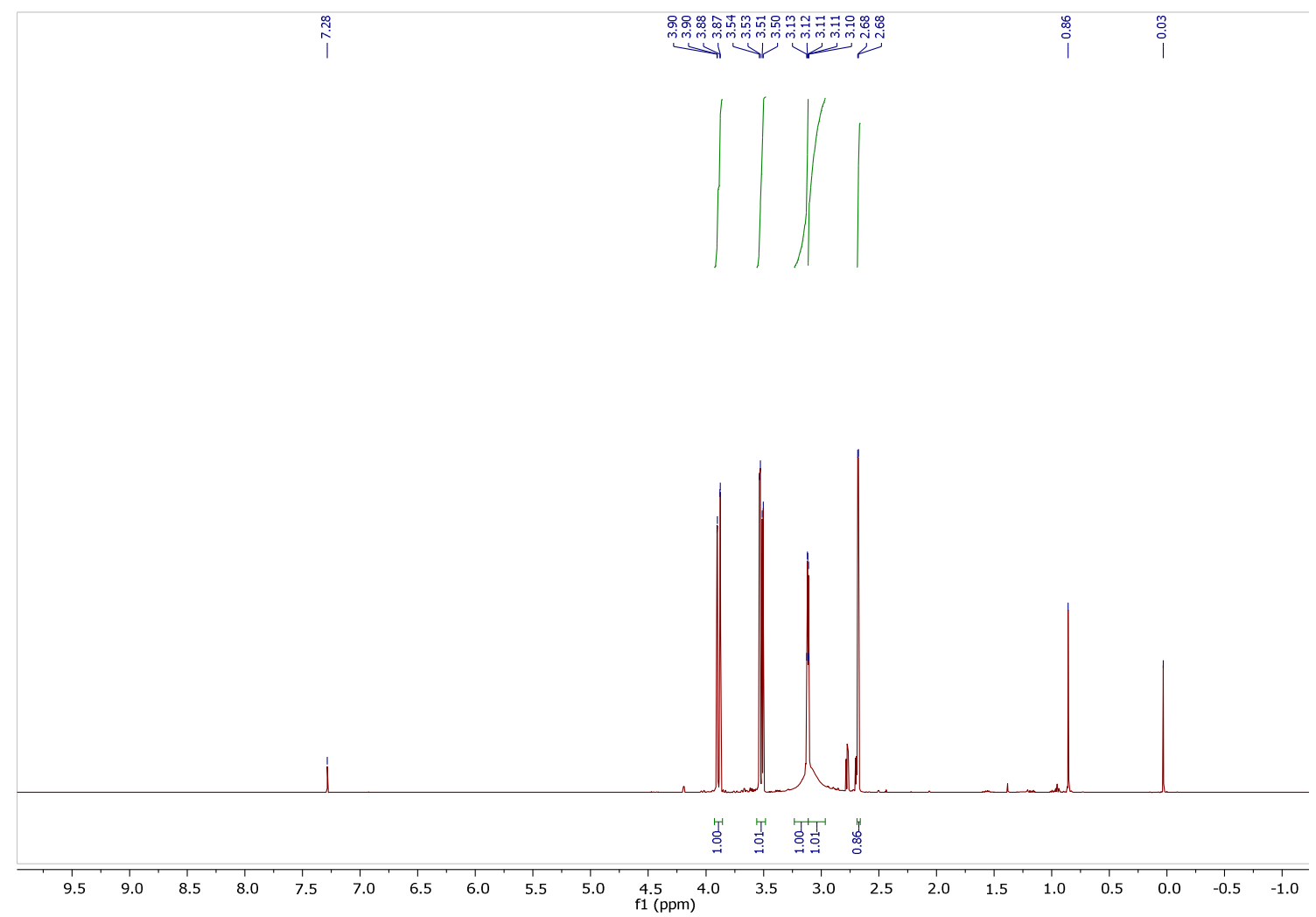

${ }^{13} \mathrm{C}-\mathrm{NMR}$ spectrum $\left(\mathrm{CDCl}_{3}\right)$

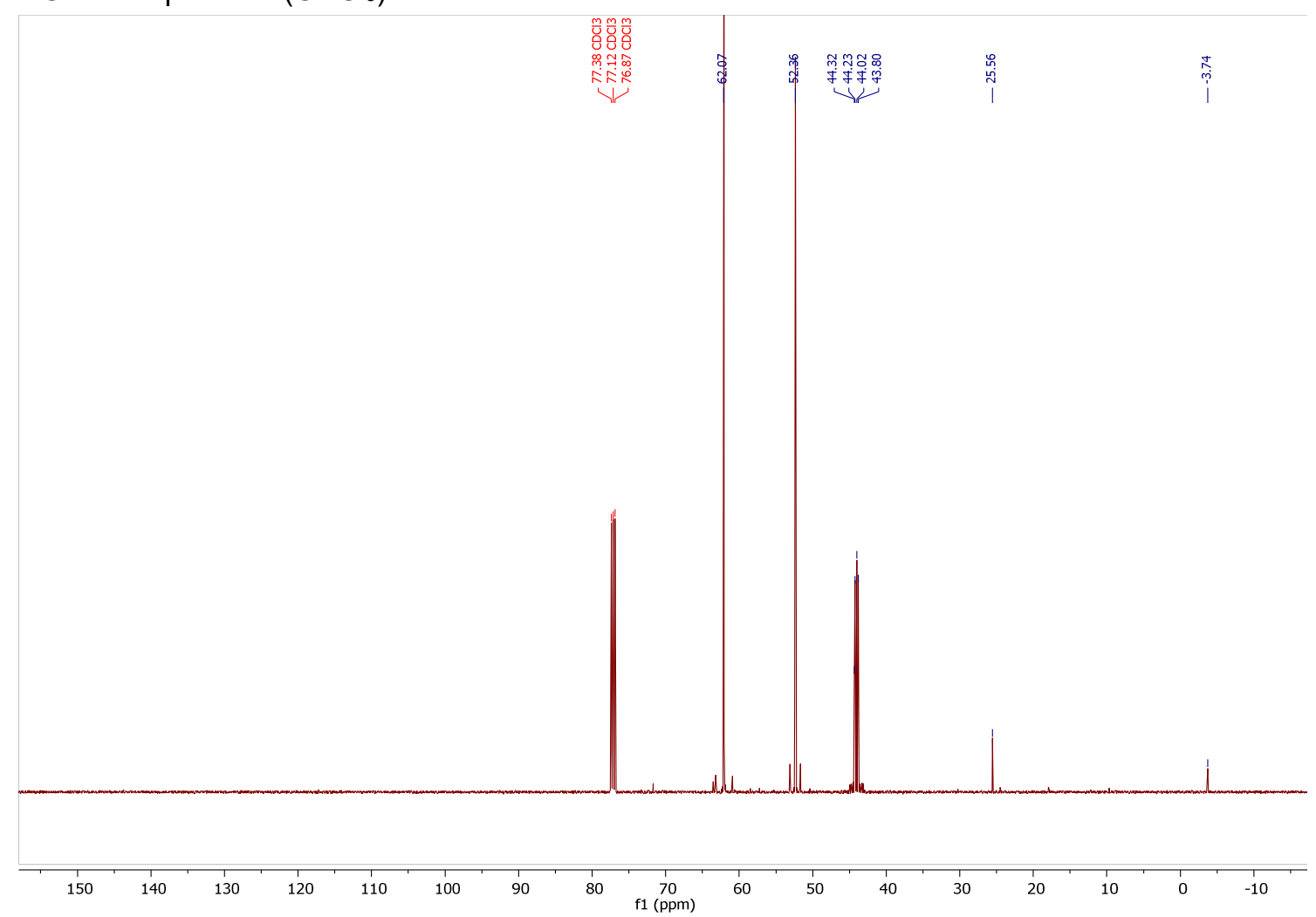


Compound F

${ }^{1} \mathrm{H}$ NMR spectrum $\left(\mathrm{CDCl}_{3}\right)$
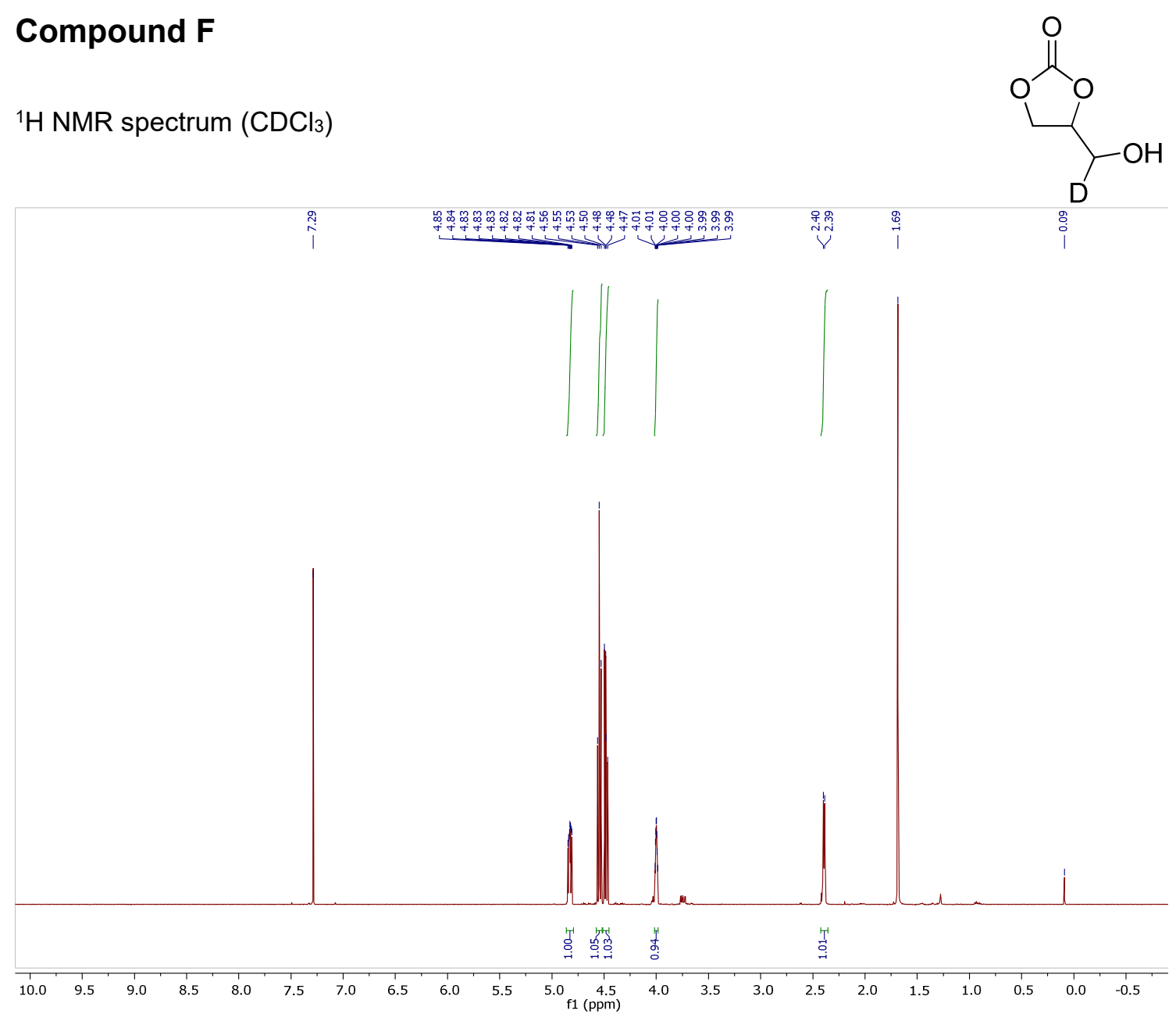

${ }^{13} \mathrm{C}$-NMR spectrum $\left(\mathrm{CDCl}_{3}\right)$

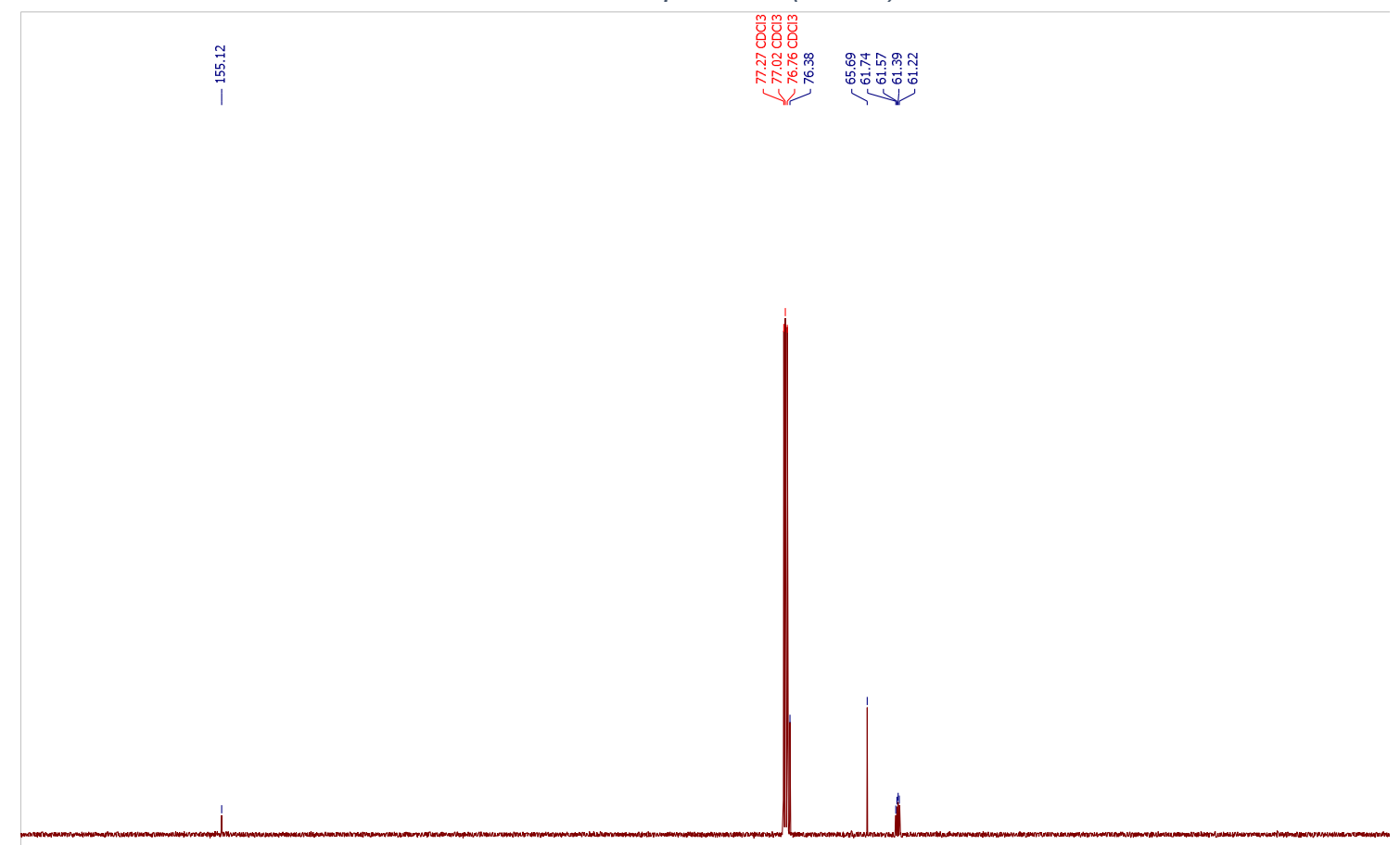

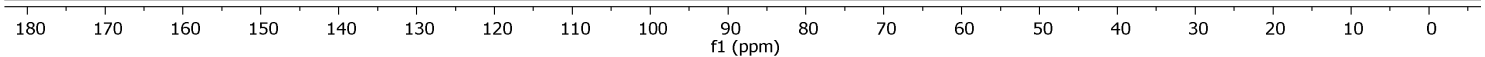




\section{References:}

[1] M. Kol, M. Shamis, I. Goldberg, Z. Goldschmidt, S. Alfi, E. Hayut-Salant, Inorg. Chem. Commun. 2001, 4, 177-179.

[2] C. J. Whiteoak, N. Kielland, V. Laserna, F. Castro-Gómez, E. Martin, E. C. EscuderoAdán, C. Bo, A. W. Kleij, Chem. Eur. J. 2014, 20, 2264-2275.

[3] D. Orain, J.-C. Guillemin, J. Org. Chem. 1999, 64, 3563-3566.

[4] Gaussian 09, Revision D.01, Frisch, M. J.; Trucks, G. W.; Schlegel, H. B.; Scuseria, G. E.; Robb, M. A.; Cheeseman, J. R.; Scalmani, G.; Barone, V.; Mennucci, B.; Petersson, G. A.; Nakatsuji, H.; Caricato, M.; Li, X.; Hratchian, H. P.; Izmaylov, A. F.; Bloino, J.; Zheng, G.; Sonnenberg, J. L.; Hada, M.; Ehara, M.; Toyota, K.; Fukuda, R.; Hasegawa, J.; Ishida, M.; Nakajima, T.; Honda, Y.; Kitao, O.; Nakai, H.; Vreven, T.; Montgomery, J. A., Jr.; Peralta, J. E.; Ogliaro, F.; Bearpark, M.; Heyd, J. J.; Brothers, E.; Kudin, K. N.; Staroverov, V. N.; Kobayashi, R.; Normand, J.; Raghavachari, K.; Rendell, A.; Burant, J. C.; Iyengar, S. S.; Tomasi, J.; Cossi, M.; Rega, N.; Millam, M. J.; Klene, M.; Knox, J. E.; Cross, J. B.; Bakken, V.; Adamo, C.; Jaramillo, J.; Gomperts, R.; Stratmann, R. E.; Yazyev, O.; Austin, A. J.; Cammi, R.; Pomelli, C.; Ochterski, J. W.; Martin, R. L.; Morokuma, K.; Zakrzewski, V. G.; Voth, G. A.; Salvador, P.; Dannenberg, J. J.; Dapprich, S.; Daniels, A. D.; Farkas, Ö.; Foresman, J. B.; Ortiz, J. V.; Cioslowski, J.; Fox, D. J. Gaussian, Inc., Wallingford CT, 2013.

[5] Chai, J.-D.; Head-Gordon, M. Phys. Chem. Chem. Phys., 2008, 10, 6615-6620.

[6] (a) Becke, A. D. J. Chem. Phys.1997, 107, 8554. (b) Schmider, H. L.; Becke, A. D.J. Chem. Phys.1998, 108, 9624. (c) Grimme, S.; Ehrlich, S.; Goerick, L. J. Comp. Chem. 2011, 32, 1456.

[7] (a) Krishnan, R.; Binkley, J. S.; Seeger, R.; Pople, J. A. J. Chem. Phys.1980, 72, 650, (b) McLean, A. D.; Chandler, G. S. J. Chem. Phys.1980, 72, 5639

[8] Martin, R. L.; Hay, P. J.; Pratt, L. R. J. Phys. Chem. A 1998, 102, 3565.

[9] NIST Computational Chemistry Comparison and Benchmark Database. NIST Standard Reference Database Number 101. Release 18, October 2016, Editor: Russell D. Johnson III. http://cccbdb.nist.gov/

[10] (a) Becke, A. D. J. Chem. Phys., 1993, 98, 5648-52. (b) Perdew, J. P.; Burke, K. and Wang, Y. Phys. Rev. B, 1996, 54, 16533-39.

[11] Becke, A. D. Phys. Rev. A, 1988, 38, 3098-100

[12] CRC Handbook of Chemistry and Physics, Lide, D.R. (ed), 84th Ed. CRC Press LLC, Florida 2003.

[13] Álvarez-Moreno, M.; de Graaf, C.; Lopez, N.; Maseras, F.; Poblet, J.M.; Bo, C. J. Chem. Inf. Model. 2015, 55, 95-103.

[14] Garand, E., Wende, T., Goebbert, D. J., Bergmann, R., Meijer, G., Neumark, D. M. \& Asmis, K. R. J. Am. Chem. Soc. 132, 849-856 (2010).

[15] L. A. Berben, Chem. Eur. J. 2015, 21, 2734-2742.

[16] C. Miceli, J. Rintjema, E. Martin, E. C. Escudero-Adán, C. Zonta, G. Licini, A. W. Kleij, ACS Catal. 2017, 7, 2367-2373.

[17] D. J. Darensbourg, J. C. Yarbrough, C. Ortiz, C. C. Fang, J. Am. Chem. Soc. 125, 7586-7591 (2003).

[18] A. Köpfer, B. Breit, Angew. Chem. Int. Ed. 2015, 54, 6913-6917.

[19] S. S. Higashibayashi, K.; Ishizu, T.; Hashimoto, K.; Shirahama, H.; Nakata, M., Synlett 2000, 1306-1308. 